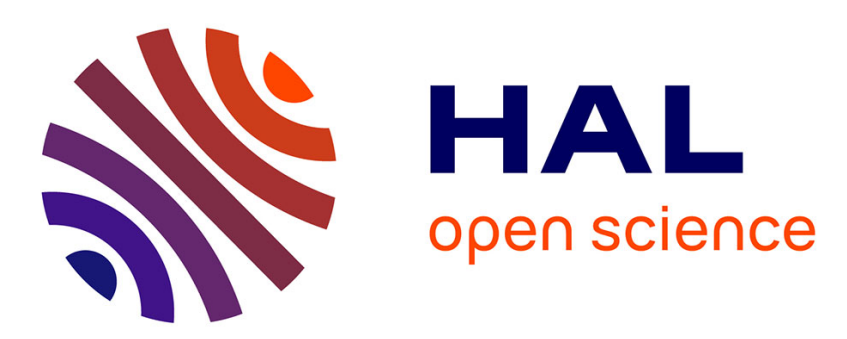

\title{
Global exact controllability of bilinear quantum systems on compact graphs and energetic controllability
}

\author{
Alessandro Duca
}

\section{To cite this version:}

Alessandro Duca. Global exact controllability of bilinear quantum systems on compact graphs and energetic controllability. SIAM Journal on Control and Optimization, 2020. hal-01875685v4

\section{HAL Id: hal-01875685 \\ https://hal.science/hal-01875685v4}

Submitted on 16 Jul 2020

HAL is a multi-disciplinary open access archive for the deposit and dissemination of scientific research documents, whether they are published or not. The documents may come from teaching and research institutions in France or abroad, or from public or private research centers.
L'archive ouverte pluridisciplinaire HAL, est destinée au dépôt et à la diffusion de documents scientifiques de niveau recherche, publiés ou non, émanant des établissements d'enseignement et de recherche français ou étrangers, des laboratoires publics ou privés. 


\title{
Global exact controllability of bilinear quantum systems on compact graphs and energetic controllability
}

\author{
Alessandro DuCA*
}

\begin{abstract}
The aim of this work is to study the controllability of the bilinear Schrödinger equation on compact graphs. In particular, we consider the equation $(B S E) i \partial_{t} \psi=-\Delta \psi+u(t) B \psi$ in the Hilbert space $L^{2}(\mathscr{G}, \mathbb{C})$, with $\mathscr{G}$ being a compact graph. The Laplacian $-\Delta$ is equipped with self-adjoint boundary conditions, $B$ is a bounded symmetric operator and $u \in L^{2}((0, T), \mathbb{R})$ with $T>0$. We provide a new technique leading to the global exact controllability of the $(B S E)$ in $D\left(|\Delta|^{s / 2}\right)$ with $s \geq 3$. Afterwards, we introduce the "energetic controllability", a weaker notion of controllability useful when the global exact controllability fails. In conclusion, we develop some applications of the main results involving for instance star graphs.
\end{abstract}

\section{Introduction}

In quantum mechanics, any state of a closed system is mathematically represented by a wave function $\psi$ in the unit sphere of a Hilbert space $\mathscr{H}$. We consider the evolution of a particle confined in a network shaped as compact graph $\mathscr{G}$ (see Figure 1) and subjected to an external field which plays the role of control.

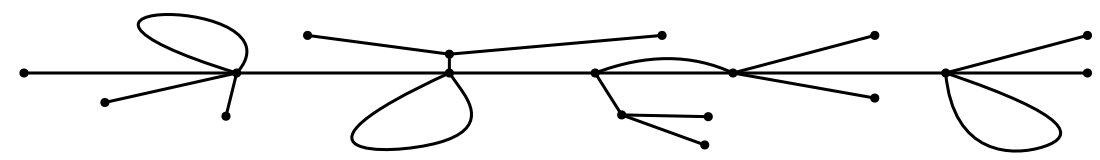

Figure 1: A compact graph is a one-dimensional domain composed by finite vertices (points) connected by edges (segments) of finite lengths.

A standard choice for such setting is to represent the action of the field by an operator $B$ and its intensity by a real function $u$. We also impose that $\mathscr{H}$ is $L^{2}(\mathscr{G}, \mathbb{C})$. The evolution of $\psi$ is modeled by the bilinear Schrödinger equation in $L^{2}(\mathscr{G}, \mathbb{C})$

$$
\left\{\begin{array}{lr}
i \partial_{t} \psi(t)=A \psi(t)+u(t) B \psi(t), & t \in(0, T) \\
\psi(0)=\psi_{0}, & T>0
\end{array}\right.
$$

The Laplacian $A=-\Delta$ is equipped with self-adjoint boundary conditions, $B$ is a bounded symmetric operator and $u \in L^{2}((0, T), \mathbb{R})$. In this context, the well-posedness of the $(B S E)$ can be deduced by the seminal work on bilinear systems [BMS82] by Ball, Mardsen and Slemrod where they show the existence of the unitary propagator $\Gamma_{t}^{u}$ generated by $A+u(t) B$.

The aim of this work is to study the controllability of the $(B S E)$ according to the structure of the graph $\mathscr{G}$, the choice of control field $B$ and the boundary conditions defining the domain of $A$.

The controllability of finite-dimensional quantum systems modeled by the $(B S E)$, when $A$ and $B$ are $N \times N$ Hermitian matrices, is well-known for being linked to the rank of the Lie algebra spanned by $A$ and $B$ (see [Alt02, Cor07]). Nevertheless the Lie algebra rank condition can not be used for infinite-dimensional quantum systems (see [Cor07]).

The global approximate controllability of the $(B S E)$ was proved with different techniques in literature. We refer to [Mir09, Ner10] for Lyapunov techniques, while we cite [BCMS12, BGRS15] for adiabatic arguments and [BdCC13, BCS14] for Lie-Galerking methods.

\footnotetext{
*Université Grenoble Alpes, CNRS, Institut Fourier, F-38000 Grenoble, France, email: alessandro.duca@univ-grenoblealpes.fr
} 
The exact controllability of infinite-dimensional quantum systems is in general a more delicate matter. When we consider the linear Schrödinger equation, the controllability and observability properties are reciprocally dual. Different results were developed by addressing directly or by duality the control problem with different techniques: multiplier methods [Lio83, Mac94], microlocal analysis [BLR92, Bur91, Leb92] and Carleman estimates [BM08, LT92, MOR08]. In any case, when one considers graphs type domains, a complete theory is far from being formulated. Indeed, the interaction between the different components of a graph may generate unexpected phenomena (see [DZ06]).

The bilinear Schrödinger equation is well-known for not being exactly controllable in the Hilbert space where it is defined when $B$ is a bounded operator and $u \in L^{2}((0, T), \mathbb{R})$ with $T>0$ (even though it is well-posed in such space). This result was proved by Turinici in [Tur00] by exploiting the techniques from the work [BMS82]. As a consequence, the exact controllability of bilinear quantum systems can not be addressed with the classical techniques valid for the linear Schrödinger equation and weaker notions of controllability are necessary. The turning point for this kind of studies has been the idea of controlling the equation in subspaces of $D(A)$ introduced by Beauchard in [Bea05]. Following this approach, different works were developed for the bilinear Schrödinger equation (BSE) with $\mathscr{G}=(0,1)$ and $A=-\Delta_{D}$ the Dirichlet Laplacian:

$$
\left.D\left(-\Delta_{D}\right)=H^{2}((0,1), \mathbb{C}) \cap H_{0}^{1}((0,1), \mathbb{C})\right), \quad-\Delta_{D} \psi:=-\Delta \psi, \quad \forall \psi \in D\left(-\Delta_{D}\right) .
$$

For instance, in [BL10], Beauchard and Laurent proved the well-posedness and the local exact controllability of the bilinear Schrödinger equation in $H_{(0)}^{s}:=D\left(\left|-\Delta_{D}\right|^{s / 2}\right)$ for $s=3$. For the global exact controllability in $H_{(0)}^{3}$, we refer to [BL17, Duc19], while we mention [Mor14, MN15] for simultaneous exact controllability results in $H_{(0)}^{3}$ and $H_{(0)}^{4}$.

Studying the controllability of the bilinear Schrödinger equation (BSE) on compact graphs presents an additional problem. In particular, when we consider $\left(\lambda_{k}\right)_{k \in \mathbb{N}^{*}}$, the ordered sequence of eigenvalues of $A$, it is possible to show that there exists $\mathcal{M} \in \mathbb{N}^{*}$ such that

$$
\inf _{k \in \mathbb{N}^{*}}\left|\lambda_{k+\mathcal{M}}-\lambda_{k}\right|>0
$$

(as ensured in [Duc18, Lemma 2.4]). Nevertheless, the uniform spectral gap $\inf _{k \in \mathbb{N}^{*}}\left|\lambda_{k+1}-\lambda_{k}\right|>0$ is only valid when $\mathscr{G}=(0,1)$. This hypothesis is crucial for the techniques developed in the works [BL10, Duc19, Mor14], which can not be directly applied without imposing further assumptions.

As far as we know, the bilinear Schrödinger equation on compact graphs has only been studied in [Duc18]. There, the author ensures that, if there exist $C>0$ and suitable $\tilde{d} \geq 0$ such that

$$
\left|\lambda_{k+1}-\lambda_{k}\right| \geq C k^{-\tilde{d}}, \quad \forall k \in \mathbb{N}^{*},
$$

then the global exact controllability of the $(B S E)$ can be guaranteed in some subspaces of $L^{2}(\mathscr{G}, \mathbb{C})$.

\section{Novelties of the work: Global exact controllability}

The aim of this work is to present a new technique ensuring the global exact controllability of the $(B S E)$ in different frameworks from the ones considered in [Duc18]. Here, we focus on discussing few interesting applications of our result involving star graphs composed by any number of edges. The general outcome is postponed to the next section (Theorem 2.3) in order to avoid further technicalities at this moment.

Let $\mathscr{G}$ be a star graph with $N \in \mathbb{N}^{*}$ edges $\left\{e_{j}\right\}_{j \leq N}$. We denote by $v$ the internal vertex of $\mathscr{G}$ and by $\left\{v_{j}\right\}_{j \leq N}$ the set of the external vertices such that $v_{j} \in e_{j}$ for every $j \leq N$. Each edge $e_{j}$ with $j \leq N$ is equipped with a coordinate going from 0 to the length of the edge $L_{j}$. We set the coordinate 0 in $v_{j}$.

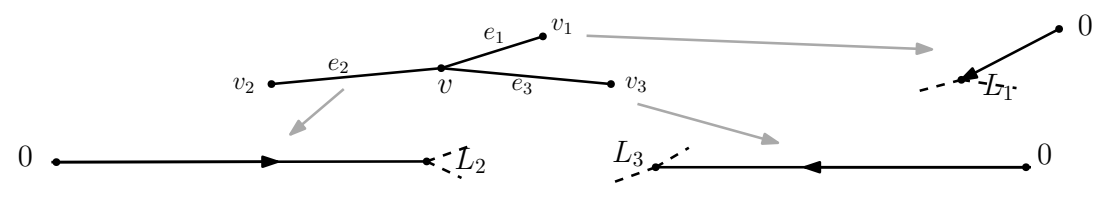

Figure 2: Parametrization of a star graph with $N=3$ edges.

We consider functions $f:=\left(f^{1}, \ldots, f^{N}\right): \mathscr{G} \rightarrow \mathbb{C}$ so that $f^{j}:\left(0, L_{j}\right) \rightarrow \mathbb{C}$ for every $j \leq N$. We denote $L^{2}(\mathscr{G}, \mathbb{C})=\prod_{j \leq N} L^{2}\left(\left(0, L_{j}\right), \mathbb{C}\right)$ the Hilbert space equipped with the norm $\|\cdot\|_{L^{2}}$. The controllability result that we present is guaranteed when the lengths $\left\{L_{j}\right\}_{j \leq N}$ satisfy suitable assumptions introduced in the following definition. 
Definition 1.1. Fixed $N \in \mathbb{N}^{*}$, we define $\mathcal{A L}(N)$ such as the set of $\left\{L_{j}\right\}_{j \leq N} \in\left(\mathbb{R}^{+}\right)^{N}$ so that the numbers $\left\{1, L_{1}, \ldots, L_{N}\right\}$ are linearly independent over $\mathbb{Q}$ and all the ratios $L_{k} / L_{j}$ are algebraic irrational numbers.

The set $\mathcal{A L}(N)$ with $N \in \mathbb{N}^{*}$ contains the uncountable set of $\left\{L_{j}\right\}_{j \leq N} \in\left(\mathbb{R}^{+}\right)^{N}$ such that each $L_{j}$ can be written in the form $t \widetilde{L}_{j}$ where all the ratios $\widetilde{L}_{j} / \widetilde{L}_{k}$ are algebraic irrational numbers and $t$ is a transcendental number. For instance, $\{\pi \sqrt{2}, \pi \sqrt{3}\}$ belongs to $\mathcal{A L}(2)$.

Definition 1.2. Let $\Gamma_{T}^{u}$ be the unitary propagator associated to the (BSE) with $u \in L^{2}((0, T), \mathbb{R})$ and $T>0$. The $(B S E)$ is globally exactly controllable in $D\left(|A|^{\frac{s}{2}}\right)$ with $s \geq 3$ when, for every $\psi^{1}, \psi^{2} \in D\left(|A|^{\frac{s}{2}}\right)$ such that $\left\|\psi^{1}\right\|_{L^{2}}=\left\|\psi^{2}\right\|_{L^{2}}$, there exist $T>0$ and $u \in L^{2}((0, T), \mathbb{R})$ such that $\Gamma_{T}^{u} \psi^{1}=\psi^{2}$.

We are finally ready to present two interesting results obtained from the techniques of this work.

Theorem 1.3. Let $\mathscr{G}$ be a star graph. Let $D(A)$ be the set of functions $f \in \prod_{j \leq N} H^{2}\left(e_{j}, \mathbb{C}\right)$ such that:

- $f^{j}(0)=0$ for every $j \leq N$ (Dirichlet boundary conditions in the external vertices $\left\{v_{j}\right\}_{j \leq N}$ );

- $f^{j}\left(L_{j}\right)=f^{k}\left(L_{k}\right)$ for every $j, k \leq N$ and $\sum_{j \leq N} \partial_{x} f^{j}\left(L_{j}\right)=0$ (Neumann-Kirchhoff conditions in $v$ ).

Let the operator $B$ be such that:

$$
B: \psi=\left(\psi^{1}, \ldots, \psi^{N}\right) \in L^{2}(\mathscr{G}, \mathbb{C}) \longmapsto\left(\left(x-L_{1}\right)^{4} \psi^{1}(x), 0, \ldots, 0\right) .
$$

There exists $\mathcal{C} \subset\left(\mathbb{R}^{+}\right)^{N}$ countable such that, for every $\left\{L_{j}\right\}_{j \leq N} \in \mathcal{A L}(N) \backslash \mathcal{C}$, the $(B S E)$ is globally exactly controllable in $D\left(|A|^{\frac{4+\epsilon}{2}}\right)$ for every $\epsilon>0$.

A similar result to Theorem 1.3 is the following. Here, the (BSE) is considered on a generic star graph equipped with Neumann boundary conditions on the external vertices instead of the Dirichlet ones.

Theorem 1.4. Let $\mathscr{G}$ be a star graph. Let $D(A)$ be the set of functions $f \in \prod_{j \leq N} H^{2}\left(e_{j}, \mathbb{C}\right)$ such that:

- $\partial_{x} f^{j}(0)=0$ for every $j \leq N$ (Neumann boundary conditions in the external vertices $\left\{v_{j}\right\}_{j \leq N}$ );

- $f^{j}\left(L_{j}\right)=f^{k}\left(L_{k}\right)$ for every $j, k \leq N$ and $\sum_{j \leq N} \partial_{x} f^{j}\left(L_{j}\right)=0$ (Neumann-Kirchhoff conditions in $v$ ).

Let the operator $B$ be such that:

$$
B: \psi=\left(\psi^{1}, \ldots, \psi^{N}\right) \in L^{2}(\mathscr{G}, \mathbb{C}) \longmapsto\left(\left(5 x^{6}-24 x^{5} L_{1}+45 x^{4} L_{1}^{2}-40 x^{3} L_{1}^{3}+15 x^{2} L_{1}^{4}-L_{1}^{6}\right) \psi^{1}(x), 0, \ldots, 0\right) .
$$

There exists $\mathcal{C} \subset\left(\mathbb{R}^{+}\right)^{N}$ countable such that, for every $\left\{L_{j}\right\}_{j \leq N} \in \mathcal{A} \mathcal{L}(N) \backslash \mathcal{C}$, the $(B S E)$ is globally exactly controllable in $D\left(|A|^{\frac{5+\epsilon}{2}}\right)$ for every $\epsilon>0$.

Theorem 1.3 and Theorem 1.4 are deduced from an abstract global exact controllability result stated in the next section in Theorem 2.3. Such theorem presents hypotheses on $\left(\lambda_{k}\right)_{k \in \mathbb{N}^{*}}$ and on the operator $B$ so that the controllability of the bilinear Schrödinger equation (BSE) is guaranteed for a general $\mathscr{G}$.

As it is common for this type of outcomes, the global exact controllability of the (BSE) can be ensured by extending a local result following from the solvability of a suitable "moment problem" (an example can be found in (8)). In order to prove Theorem 1.3 and Theorem 1.4, we start by studying assumptions on $\left(\lambda_{k}\right)_{k \in \mathbb{N}^{*}}$ and on the operator $B$ leading to the solvability of such moment problem. In a second moment, we prove the abstract global exact controllability result of Theorem 2.3. Afterwards, we ensure the validity of the spectral assumptions considered in Theorem 2.3 for suitable star graphs. In conclusion, we validate the remaining hypotheses when the operator $B$ is defined as in Theorem 1.3 or Theorem 1.4.

The main novelty of Theorem 1.3 and Theorem 1.4 is the validity of the controllability results when $\mathscr{G}$ is a star graph with any number of edges. In fact, the techniques developed in the existing work [Duc18] only allow to consider star graphs with at most 4 edges (see [Duc18, Proposition 3.3]). In addition, the controllability is guaranteed even though the control field $B$ only acts on one edges of the graph, which is due to the choice of the lengths $\left\{L_{j}\right\}_{j \leq N}$ in $\mathcal{A L}(N)$. About this fact, if some ratios $L_{k} / L_{1}$ are rationals, then the spectrum of the operator $A$ presents multiple eigenvalues and there exist eigenfunctions of $A$ vanishing in $e_{1}$ (we refer to Remark 5.3 for further details on this fact). As a consequence, the dynamics of the bilinear Schrödinger equation (BSE) stabilizes such eigenfunctions since the control operator $B$ only acts on $e_{1}$. This is an obvious obstruction to the controllability which underlines the importance of choosing suitable lengths for the edges of the graph in this kind of problems.

\section{Novelties of the work: Energetic controllability}

In the spirit of the results provided in [BC06], we introduce a weaker notion of controllability: the energetic controllability. 
Definition 1.5. Let $\left(\varphi_{k}\right)_{k \in \mathbb{N}^{*}}$ be an orthonormal system of $L^{2}(\mathscr{G}, \mathbb{C})$ (not necessarily complete) composed by eigenfunctions of $A$ and $\left(\mu_{k}\right)_{k \in \mathbb{N}^{*}}$ be the corresponding eigenvalues. Let $\Gamma_{T}^{u}$ be the unitary propagator associated to the $(\mathrm{BSE})$ with $u \in L^{2}((0, T), \mathbb{R})$ and $T>0$. The $(B S E)$ is energetically controllable in $\left(\mu_{k}\right)_{k \in \mathbb{N}^{*}}$ if, for every $m, n \in \mathbb{N}^{*}$, there exist $T>0$ and $u \in L^{2}((0, T), \mathbb{R})$ so that $\Gamma_{T}^{u} \varphi_{m}=\varphi_{n}$.

The energetic controllability guarantees the controllability of specific energy levels of the quantum system $i \partial_{t} \psi=A \psi$ in $L^{2}(\mathscr{G}, \mathbb{C})$ via the external field $u(t) B$. An application of the abstract energetic controllability result, which is presented in Section 6 (in Theorem 6.1), is the following theorem.

Theorem 1.6. Let $\mathscr{G}$ be a star graph with edges of equal length $L$. Let $D(A)$ be defined such as in Theorem 1.3. Let the operator $B$ be such that:

$$
B: \psi=\left(\psi^{1}, \ldots, \psi^{N}\right) \in L^{2}(\mathscr{G}, \mathbb{C}) \longmapsto\left((x-L)^{2} \psi^{1}(x), 0, \ldots, 0\right) .
$$

The $(B S E)$ is energetically controllable in $\left(\frac{k^{2} \pi^{2}}{4 L^{2}}\right)_{k \in \mathbb{N}^{*}}$.

Theorem 1.6 is valid although the spectrum of $A$ presents multiple eigenvalues and then the global exact controllability from Theorem 2.3 is not satisfied (also [Duc18, Theorem 3.2] is not guaranteed). In addition, the energetic controllability is ensured with respect to all the energy levels of the quantum system $i \partial_{t} \psi=A \psi$, since the eigenvalues of $A$ are $\left(\frac{k^{2} \pi^{2}}{4 L^{2}}\right)_{k \in \mathbb{N}^{*}}$ (without considering their multiplicity).

The energetic controllability is useful when it is not possible to fully characterize the spectrum of $A$ because of the complexity of the graph $\mathscr{G}$. By studying the structure of $\mathscr{G}$, it is possible to explicit some eigenvalues $\left(\mu_{k}\right)_{k \in \mathbb{N}^{*}}$ and verify if the system is energetically controllable in $\left(\mu_{k}\right)_{k \in \mathbb{N}^{*}}$. In Section 6.1 , we discuss some examples where the result is satisfied, e.g graphs containing loops as in Figure 3 (a loop is an edge of the graph which is connected from both extremes to the same vertex).

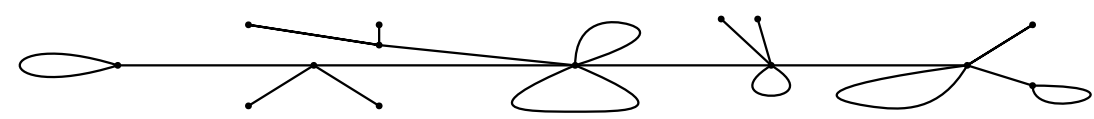

Figure 3: Example of compact graph containing more loops.

\section{Scheme of the work}

In Section 2, we present the main assumptions adopted in the work, the well-posedness of the $(B S E)$ in specific subspaces of $L^{2}(\mathscr{G}, \mathbb{C})$ and the abstract global exact controllability result in Theorem 2.3.

In Section 3, we study the solvability of the "moment problem" under the hypotheses of Theorem 2.3. In Section 4, we prove Theorem 2.3 by extending a local exact controllability result provided in Proposition 4.2. To the purpose, we use the outcomes developed in Section 3 and Appendix $A$.

In Section 5, we study the (BSE) on star graphs and we prove Theorem 1.3 and Theorem 1.4.

In Section 6, we discuss the energetic controllability of the (BSE) and we prove Theorem 1.6.

In Appendix $A$, we present the global approximate controllability of the bilinear Schrödinger equation.

In Appendix $B$, we study some spectral results adopted in the work.

\section{The bilinear Schrödinger equation on compact graphs}

\subsection{Preliminaries}

Let $\mathscr{G}$ be a compact graph composed by $N \in \mathbb{N}^{*}$ edges $\left\{e_{j}\right\}_{j \leq N}$ of lengths $\left\{L_{j}\right\}_{j \leq N}$ and $M \in \mathbb{N}^{*}$ vertices $\left\{v_{j}\right\}_{j \leq M}$. For every vertex $v$, we denote $N(v):=\left\{l \in\{1, \ldots, N\} \mid v \in e_{l}\right\}$ and $n(v):=|N(v)|$. We call $V_{e}$ and $V_{i}$ the external and the internal vertices of $\mathscr{G}$, i.e.

$$
V_{e}:=\left\{v \in\left\{v_{j}\right\}_{j \leq M} \mid \exists ! e \in\left\{e_{j}\right\}_{j \leq N}: v \in e\right\}, \quad V_{i}:=\left\{v_{j}\right\}_{j \leq M} \backslash V_{e} .
$$

We study graphs equipped with a metric, which parametrizes each edge $e_{j}$ with a coordinate going from 0 to its length $L_{j}$. A graph is compact when it is composed by a finite number of vertices and edges of finite lengths. We consider functions $f:=\left(f^{1}, \ldots, f^{N}\right): \mathscr{G} \rightarrow \mathbb{C}$ with domain a compact metric graph $\mathscr{G}$ so that $f^{j}: e_{j} \rightarrow \mathbb{C}$ for every $j \leq N$. We denote

$$
\mathscr{H}=L^{2}(\mathscr{G}, \mathbb{C})=\prod_{j \leq N} L^{2}\left(e_{j}, \mathbb{C}\right) .
$$


The Hilbert space $\mathscr{H}$ is equipped with the norm $\|\cdot\|_{L^{2}}$ induced by the scalar product

$$
\langle\psi, \varphi\rangle_{L^{2}}:=\sum_{j \leq N}\left\langle\psi^{j}, \varphi^{j}\right\rangle_{L^{2}\left(e_{j}, \mathbb{C}\right)}=\sum_{j \leq N} \int_{e_{j}} \overline{\psi^{j}}(x) \varphi^{j}(x) d x, \quad \forall \psi, \varphi \in \mathscr{H} .
$$

For $s>0$, we define the spaces

$$
\begin{gathered}
H^{s}=H^{s}(\mathscr{G}, \mathbb{C}):=\prod_{j=1}^{N} H^{s}\left(e_{j}, \mathbb{C}\right), \\
h^{s}=\left\{\left.\left(x_{j}\right)_{j \in \mathbb{N}^{*}} \subset \mathbb{C}\left|\sum_{j=1}^{\infty}\right| j^{s} x_{j}\right|^{2}<\infty\right\} .
\end{gathered}
$$

We equip $h^{s}$ with the norm $\left\|\left(x_{j}\right)_{j \in \mathbb{N}^{*}}\right\|_{(s)}=\left(\sum_{j=1}^{\infty}\left|j^{s} x_{j}\right|^{2}\right)^{\frac{1}{2}}$ for every $\left(x_{j}\right)_{j \in \mathbb{N}^{*}} \in h^{s}$.

Let $f=\left(f^{1}, \ldots, f^{N}\right): \mathscr{G} \rightarrow \mathbb{C}$ be smooth and $v$ be a vertex of $\mathscr{G}$ connected once to an edge $e_{j}$ with $j \leq N$. When the coordinate parametrizing $e_{j}$ in the vertex $v$ is equal to 0 (resp. $L_{j}$ ), we denote

$$
\partial_{x} f^{j}(v)=\partial_{x} f^{j}(0), \quad\left(\text { resp. } \partial_{x} f^{j}(v)=-\partial_{x} f^{j}\left(L_{j}\right)\right) .
$$

When $e_{j}$ is a loop and it is connected to $v$ in both of its extremes, we use the notation

$$
\partial_{x} f^{j}(v)=\partial_{x} f^{j}(0)-\partial_{x} f^{j}\left(L_{j}\right)
$$

When $v$ is an external vertex and then $e_{j}$ is the only edge connected to $v$, we call $\partial_{x} f(v)=\partial_{x} f^{j}(v)$.

In the bilinear Schrödinger equation $(B S E)$, we consider the Laplacian $A$ being self-adjoint and we denote $\mathscr{G}$ as quantum graph. From now on, when we introduce a quantum graph $\mathscr{G}$, we implicitly define on $\mathscr{G}$ a self-adjoint Laplacian $A$. Formally, $D(A)$ is characterized by the following boundary conditions.

Boundary conditions. Let $\mathscr{G}$ be a compact quantum graph.

$(\mathcal{D})$ A vertex $v \in V_{e}$ is equipped with Dirichlet boundary conditions when $f(v)=0$ for every $f \in D(A)$.

$(\mathcal{N})$ A vertex $v \in V_{e}$ is equipped with Neumann boundary conditions when $\partial_{x} f(v)=0$ for every $f \in D(A)$.

$(\mathcal{N K})$ A vertex $v \in V_{i}$ is equipped with Neumann-Kirchhoff boundary conditions when every $f \in D(A)$ is continuous in $v$ and $\sum_{j \in N(v)} \partial_{x} f^{j}(v)=0$ (we refer to (3) and (4) for further details on the notation).

Notations. Let $\mathscr{G}$ be a compact quantum graph.

- The graph $\mathscr{G}$ is said to be equipped with $(\mathcal{D})(\operatorname{resp} .(\mathcal{N}))$ when every $v \in V_{e}$ is equipped with $(\mathcal{D})$ (resp. $(\mathcal{N}))$ and every $v \in V_{i}$ with $(\mathcal{N K})$.

- The graph $\mathscr{G}$ is said to be equipped with $(\mathcal{D} / \mathcal{N})$ when every $v \in V_{e}$ is equipped with $(\mathcal{D})$ or $(\mathcal{N})$, while every $v \in V_{i}$ with $(\mathcal{N K})$.

When the boundary conditions described above are satisfied, the Laplacian $A$ is self-adjoint (see [Kuc04, Theorem 3] for further details) and admits purely discrete spectrum (see [Kuc04, Theorem 18]). We denote by $\left(\lambda_{k}\right)_{k \in \mathbb{N}^{*}}$ the ordered sequence of eigenvalues of $A$ and we define a Hilbert basis of $\mathscr{H}$ :

$$
\Phi:=\left(\phi_{k}\right)_{k \in \mathbb{N}^{*}}
$$

composed by corresponding eigenfunctions. From [Duc18, Lemma 2.3], there exist $C_{1}, C_{2}>0$ so that

$$
C_{1} k^{2} \leq \lambda_{k} \leq C_{2} k^{2}, \quad \forall k \geq 2
$$

Let $[r]$ be the entire part of a number $r \in \mathbb{R}$. For $s>0$, we denote

$$
\begin{gathered}
H_{\mathcal{N} \mathcal{K}}^{s}:=\left\{\psi \in H^{s}(\mathscr{G}, \mathbb{C}) \mid \partial_{x}^{2 n} \psi \text { is continuous in } v, \forall n \in \mathbb{N}, n<[(s+1) / 2], \forall v \in V_{i} ;\right. \\
\left.\sum_{j \in N(v)} \partial_{x}^{2 n+1} \psi^{j}(v)=0, \forall n \in \mathbb{N}, n<[s / 2], \forall v \in V_{i}\right\}, \\
H_{\mathscr{G}}^{s}:=D\left(A^{s / 2}\right), \quad \quad\|\cdot\|_{(s)}:=\|\cdot\|_{H_{\mathscr{G}}^{s}}=\left(\sum_{k \in \mathbb{N}^{*}}\left|k^{s}\left\langle\cdot, \phi_{k}\right\rangle_{L^{2}}\right|^{2}\right)^{\frac{1}{2}} .
\end{gathered}
$$

We introduce the main assumptions adopted in the manuscript by considering $\left(\mu_{k}\right)_{k \in \mathbb{N}^{*}} \subseteq\left(\lambda_{k}\right)_{k \in \mathbb{N}^{*}}$ an ordered sequence of some eigenvalues of $A$ and the corresponding eigenfunctions

$$
\varphi:=\left(\varphi_{k}\right)_{k \in \mathbb{N}^{*}} \subseteq\left(\phi_{k}\right)_{k \in \mathbb{N}^{*}} .
$$

Let $\eta>0, a \geq 0, I:=\left\{(j, k) \in\left(\mathbb{N}^{*}\right)^{2}: j<k\right\}$ and $\widetilde{\mathscr{H}}:=\overline{\operatorname{span}\left\{\varphi_{k} \mid k \in \mathbb{N}^{*}\right\}}{ }^{2}$. 
Assumptions I $(\varphi, \eta)$. The bounded symmetric operator $B$ satisfies the following conditions.

1. There exists $C>0$ such that $\left|\left\langle\varphi_{k}, B \varphi_{1}\right\rangle_{L^{2}}\right| \geq \frac{C}{k^{2+\eta}}$ for every $k \in \mathbb{N}^{*}$.

2. For every $(j, k),(l, m) \in I$ such that $(j, k) \neq(l, m)$ and $\mu_{j}-\mu_{k}-\mu_{l}+\mu_{m}=0$, it holds

$$
\left\langle\varphi_{j}, B \varphi_{j}\right\rangle_{L^{2}}-\left\langle\varphi_{k}, B \varphi_{k}\right\rangle_{L^{2}}-\left\langle\varphi_{l}, B \varphi_{l}\right\rangle_{L^{2}}+\left\langle\varphi_{m}, B \varphi_{m}\right\rangle_{L^{2}} \neq 0
$$

Assumptions I $(\eta)$. The couple $(A, B)$ satisfies Assumptions $\mathrm{I}(\Phi, \eta)$ with $\Phi$ a Hilbert basis of $\mathscr{H}$ made by eigenfunctions of $A$.

The first condition of Assumptions $\mathrm{I}(\varphi, \eta)$ (resp. Assumptions $\mathrm{I}(\eta)$ ) quantifies how much $B$ mixes the eigenspaces associated to the eigenfunctions $\left(\varphi_{k}\right)_{k \in \mathbb{N}^{*}}\left(\operatorname{resp} .\left(\phi_{k}\right)_{k \in \mathbb{N}^{*}}\right)$. This assumption is crucial for the controllability. Indeed, when $B$ stabilizes such spaces, also $\Gamma_{T}^{u}$ does the same and we can not expect to obtain controllability results. The second hypothesis is used to decouple some eigenvalues resonances appearing in the proof of the approximate controllability that we use in order to prove our main results.

Assumptions II $(\varphi, \eta, a)$. Let $\operatorname{Ran}\left(\left.B\right|_{H_{\mathscr{G}}^{2} \cap \widetilde{\mathscr{H}}}\right) \subseteq H_{\mathscr{G}}^{2} \cap \widetilde{\mathscr{H}}$ and one of the following points be satisfied.

1. When $\mathscr{G}$ is equipped with $(\mathcal{D} / \mathcal{N})$ and $a+\eta \in(0,3 / 2)$, there exists $d \in[\max \{a+\eta, 1\}, 3 / 2)$ so that

$$
\operatorname{Ran}\left(\left.B\right|_{H_{\mathscr{G}}^{2+d} \cap \widetilde{\mathscr{H}}}\right) \subseteq H^{2+d} \cap H_{\mathscr{G}}^{2} \cap \widetilde{\mathscr{H}}
$$

2. When $\mathscr{G}$ is equipped with $(\mathcal{N})$ and $a+\eta \in(0,7 / 2)$, there exist $d \in[\max \{a+\eta, 2\}, 7 / 2)$ and $d_{1} \in(d, 7 / 2)$ such that

$$
\operatorname{Ran}\left(\left.B\right|_{H_{\mathcal{N} \mathcal{K}}^{d_{1}} \cap \widetilde{\mathscr{H}}}\right) \subseteq H_{\mathcal{N} \mathcal{K}}^{d_{1}} \cap \widetilde{\mathscr{H}}, \quad \operatorname{Ran}\left(\left.B\right|_{H_{\mathscr{G}}^{2+d} \cap \widetilde{\mathscr{H}}}\right) \subseteq H^{2+d} \cap H_{\mathcal{N K}}^{1+d} \cap H_{\mathscr{G}}^{2} \cap \widetilde{\mathscr{H}}
$$

3. When $\mathscr{G}$ is equipped with $(\mathcal{D})$ and $a+\eta \in(0,5 / 2)$, there exists $d \in[\max \{a+\eta, 1\}, 5 / 2)$ such that

$$
\operatorname{Ran}\left(\left.B\right|_{H_{\mathscr{G}}^{2+d} \cap \widetilde{\mathscr{H}}}\right) \subseteq H^{2+d} \cap H_{\mathcal{N K}}^{1+d} \cap H_{\mathscr{G}}^{2} \cap \widetilde{\mathscr{H}} .
$$

If $d \geq 2$, then there exists $d_{1} \in(d, 5 / 2)$ such that $\operatorname{Ran}\left(\left.B\right|_{H^{d_{1}} \cap \widetilde{\mathscr{H}}}\right) \subseteq H^{d_{1}} \cap \widetilde{\mathscr{H}}$.

Assumptions II $(\eta, a)$. The couple $(A, B)$ satisfies Assumptions $\operatorname{II}(\Phi, \eta, a)$ with $\Phi$ a Hilbert basis of $\mathscr{H}$ made by eigenfunctions of $A$.

We introduce Assumptions $\operatorname{II}(\varphi, \eta, a)$ and Assumptions $\operatorname{II}(\varphi, \eta)$ since the choice of the boundary conditions defining $D(A)$ affects the definition of the spaces $H_{\mathscr{G}}^{s}=D\left(|A|^{\frac{s}{2}}\right)$ with $s>0$. For this reason, we have to calibrate the regularity of the control potential $B$ according to such choice.

\subsection{Well-posedness}

Now, we cite [Duc18, Theorem 4.1] where the well-posedness of the bilinear Schrödinger equation $(B S E)$ is ensured in $H_{\mathscr{G}}^{s}$ with suitable $s \geq 3$.

Proposition 2.1. [Duc18, Theorem 4.1] Let $\mathscr{G}$ be a compact quantum graph and $(A, B)$ satisfy Assumptions $I I(\eta, \tilde{d})$ with $\eta>0$ and $\tilde{d} \geq 0$. For any $T>0$ and $u \in L^{2}((0, T), \mathbb{R})$, the flow of the (BSE) is unitary in $\mathscr{H}$ and, for any initial data $\psi^{0} \in H_{\mathscr{G}}^{2+d}$ with $d$ from Assumptions $I I(\eta, \tilde{d})$, there exists a unique mild solution of (BSE) in $H_{\mathscr{G}}^{2+d}$, i.e. a function $\psi \in C^{0}\left([0, T], H_{\mathscr{G}}^{2+d}\right)$ such that

$$
\psi(t, x)=e^{-i A t} \psi^{0}(x)-i \int_{0}^{t} e^{-i A(t-s)} u(s) B \psi(s, x) d s, \quad \forall t \in[0, T] .
$$

Remark 2.2. Let $\varphi:=\left(\varphi_{k}\right)_{k \in \mathbb{N}^{*}} \subseteq\left(\phi_{k}\right)_{k \in \mathbb{N}^{*}}$ be an orthonormal system of $\mathscr{H}$ made by eigenfunctions of $A$ and $\widetilde{\mathscr{H}}:=\overline{\operatorname{span}\left\{\varphi_{k} \mid k \in \mathbb{N}^{*}\right\}}{ }^{2}$. We notice that the statement of Proposition 2.1 can be ensured in $\widetilde{\mathscr{H}}$ as the propagator $\Gamma_{t}^{u}$ preserves the space $H_{\mathscr{G}}^{2} \cap \widetilde{\mathscr{H}}$ when $B: H_{\mathscr{G}}^{2} \cap \widetilde{\mathscr{H}} \longrightarrow H_{\mathscr{G}}^{2} \cap \widetilde{\mathscr{H}}$. Thus, if $(A, B)$ satisfies Assumptions $I I(\varphi, \eta, \tilde{d})$ with $\eta>0$ and $\tilde{d} \geq 0$, then, for every $\psi^{0} \in H_{\mathscr{G}}^{2+d} \cup \widetilde{\mathscr{H}}$ with $d$ from Assumptions $I I(\varphi, \eta, \tilde{d})$ and $u \in L^{2}((0, T), \mathbb{R})$, there exists a unique mild solution of the $(B S E)$ in $H_{\mathscr{G}}^{2+d} \cup \widetilde{\mathscr{H}}$. 


\subsection{Abstract global exact controllability result}

In the following theorem, we provide the main abstract result of the work regarding the global exact controllability of the $(B S E)$.

Theorem 2.3. Let $\mathscr{G}$ be a compact quantum graph and $\left(\lambda_{k}\right)_{k \in \mathbb{N}^{*}}$ be the ordered sequence of eigenvalues of $A$. Let the following hypotheses be satisfied.

- The eigenvalues $\left(\lambda_{k}\right)_{k \in \mathbb{N}^{*}}$ are simple. There exists an entire function $G \in L^{\infty}(\mathbb{R}, \mathbb{R})$ such that there exist $J, I>0$ such that $|G(z)| \leq J e^{I|z|}$ for every $z \in \mathbb{C}$ and the numbers $\left\{ \pm \sqrt{\lambda_{k}}\right\}_{k \in \mathbb{N}^{*}}$ are simple zeros of $G$. In addition, there exist $\tilde{d} \geq 0$ and $C>0$ such that

$$
\left|G^{\prime}\left( \pm \sqrt{\lambda_{k}}\right)\right| \geq \frac{C}{k^{1+\tilde{d}}}, \quad \forall k \in \mathbb{N}^{*}
$$

- The couple $(A, B)$ satisfy Assumptions $I(\eta)$ and Assumptions $I I(\eta, \tilde{d})$ for $\eta>0$.

Then, the $(B S E)$ is globally exactly controllable in $H_{\mathscr{G}}^{2+d}$ with d from Assumptions $I I(\eta, \tilde{d})$.

Proof. See Section 4.

The proof of Theorem 2.3 is obtained by extending a local exact controllability result which follows from the solvability of a suitable moment problem in a specific subspace of $\ell^{2}(\mathbb{C})$. In other words, we ensure that, for every $\left(x_{k}\right)_{k \in \mathbb{N}^{*}}$ in such subspace, there exists $u \in L^{2}((0, T), \mathbb{R})$ with $T>0$ such that

$$
x_{k}=-i \int_{0}^{T} u(\tau) e^{i\left(\lambda_{k}-\lambda_{1}\right) \tau} d \tau, \quad \forall k \in \mathbb{N}^{*} .
$$

In the next section, we develop a new technique leading to such result when the hypotheses of Theorem 2.3 are satisfied. In particular, the entire function $G$ is used to construct the control $u$ satisfying (8) thanks to the Paley-Wiener's Theorem. The lower bound (7) provides the regularity of the subspace of $\ell^{2}(\mathbb{C})$ where we can consider $\left(x_{k}\right)_{k \in \mathbb{N}^{*}}$ in the moment problem (8).

In Section 5, we show how to construct an entire function $G$ satisfying the hypothesis of Theorem 2.3 when $\mathscr{G}$ is an appropriate star graph. In such framework, it is possible to see the numbers $\left\{ \pm \sqrt{\lambda_{k}}\right\}_{k \in \mathbb{N}^{*}}$ as the zeros of a specific function that we use to define $G$.

Remark. When $\mathscr{G}=(0,1)$, Ingham's type theorems lead to the solvability of (8) for sequences $\left(x_{k}\right)_{k \in \mathbb{N}^{*} \in}$ $\ell^{2}(\mathbb{C})$. Such techniques are valid thanks to the spectral gap $\inf _{k \in \mathbb{N}^{*}}\left|\lambda_{k+1}-\lambda_{k}\right|>0$ as explained in [BL10, Appendix B]. When $\mathscr{G}$ is a compact graph and the weak spectral gap (2) is satisfied with suitable $\tilde{d}>0$, such result can be ensured for $\left(x_{k}\right)_{k \in \mathbb{N}^{*}} \in h^{\tilde{d}(\mathcal{M}-1)}$ with $\mathcal{M}$ from (1) (see [Duc18, Appendix B]).

\section{Trigonometric moment problems}

\subsection{On the solvability of the moment problem}

The aim of this section is to prove the next proposition which ensures the solvability of the moment problem (8) when the hypotheses of Theorem 2.3 are valid.

Proposition 3.1. Let $\left(\lambda_{k}\right)_{k \in \mathbb{N}^{*}} \subset \mathbb{R}^{+}$be an ordered sequence of pairwise distinct numbers so that there exist $\delta, C_{1}, C_{2}>0$ and $\mathcal{M} \in \mathbb{N}^{*}$ such that $\inf _{k \in \mathbb{N}^{*}}\left|\sqrt{\lambda_{k+\mathcal{M}}}-\sqrt{\lambda_{k}}\right| \geq \delta \mathcal{M}$ and

$$
C_{1} k^{2} \leq \lambda_{k} \leq C_{2} k^{2}, \quad \forall k \geq 2 \text {. }
$$

Let $G$ be an entire function so that $\left\{ \pm \sqrt{\lambda_{k}}\right\}_{k \in \mathbb{N}^{*}}$ are its simple zeros, $G \in L^{\infty}(\mathbb{R}, \mathbb{R})$ and there exist $J, I>0$ such that $|G(z)| \leq J e^{I|z|}$ for every $z \in \mathbb{C}$. If there exist $\tilde{d} \geq 0$ and $C>0$ such that $\left|G^{\prime}\left( \pm \sqrt{\lambda_{k}}\right)\right| \geq \frac{C}{k^{1+\tilde{d}}}$ for every $k \in \mathbb{N}^{*}$, then for $T>2 \pi / \delta$ and for every $\left(x_{k}\right)_{k \in \mathbb{N}^{*}} \in h^{\tilde{d}}\left(\mathbb{N}^{*}, \mathbb{C}\right)$ with $x_{1} \in \mathbb{R}$, there exists $u \in L^{2}((0, T), \mathbb{R})$ so that

$$
x_{k}=\int_{0}^{T} u(\tau) e^{i\left(\lambda_{k}-\lambda_{1}\right) \tau} d \tau, \quad \forall k \in \mathbb{N}^{*} .
$$

The proof of Proposition 3.1 is provided in Section 3.5 by gathering some technical results developed below. We suggest to the uninterested reader to skip it and pass to the Section 4. 
Remark 3.2. Let the hypotheses of Proposition 3.1 be guaranteed. From Proposition 3.1, for $T>2 \pi / \delta$ and for every $\left(x_{k}\right)_{k \in \mathbb{N}^{*}} \in h^{\tilde{d}}\left(\mathbb{N}^{*}, \mathbb{C}\right)$ with $x_{1} \in \mathbb{R}$, there exists $u \in L^{2}((0, T), \mathbb{R})$ such that

$$
x_{k}=\int_{0}^{T} u(\tau) e^{-i\left(\lambda_{k}-\lambda_{1}\right) \tau} d \tau, \quad \forall k \in \mathbb{N}^{*}
$$

Indeed, for every $\left(x_{k}\right)_{k \in \mathbb{N}^{*}} \in h^{\tilde{d}}\left(\mathbb{N}^{*}, \mathbb{C}\right)$ with $x_{1} \in \mathbb{R}$, there exists $u \in L^{2}((0, T), \mathbb{R})$ such that $\overline{x_{k}}=$ $\int_{0}^{T} u(\tau) e^{i\left(\lambda_{k}-\lambda_{1}\right) \tau} d \tau$ for every $k \in \mathbb{N}^{*}$. The claim is proved by conjugating the last expression.

\subsection{Families of functions in a Hilbert space}

Let $\mathbb{Z}^{*}=\mathbb{Z} \backslash\{0\}$. We denote by $\langle\cdot, \cdot\rangle_{L^{2}(0, T)}$ the scalar product in $L^{2}((0, T), \mathbb{C})$ with $T>0$.

Definition 3.3. Let $\left(f_{k}\right)_{k \in \mathbb{Z}^{*}}$ be a family of functions in $L^{2}((0, T), \mathbb{C})$ with $T>0$. The family $\left(f_{k}\right)_{k \in \mathbb{Z}^{*}}$ is said to be minimal if and only if $f_{k} \notin \overline{\operatorname{span}\left\{f_{j}: j \neq k\right\}} L^{2}$ for every $k \in \mathbb{Z}^{*}$.

Definition 3.4. A biorthogonal family to $\left(f_{k}\right)_{k \in \mathbb{Z}^{*}} \subset L^{2}((0, T), \mathbb{C})$ is a sequence of functions $\left(g_{k}\right)_{k \in \mathbb{Z}^{*}}$ in $L^{2}((0, T), \mathbb{C})$ such that $\left\langle f_{k}, g_{j}\right\rangle_{L^{2}(0, T)}=\delta_{k, j}$ for every $k, j \in \mathbb{Z}^{*}$.

Remark 3.5. When $\left(f_{k}\right)_{k \in \mathbb{Z}^{*}}$ is minimal, there exists an unique biorthogonal family $\left(g_{k}\right)_{k \in \mathbb{Z}^{*}}$ to $\left(f_{k}\right)_{k \in \mathbb{Z}^{*}}$ belonging to $X:=\overline{\operatorname{span}\left\{f_{j}: j \in \mathbb{Z}^{*}\right\}}{ }^{2}$. Indeed, $\left(g_{k}\right)_{k \in \mathbb{Z}^{*}}$ can be constructed by setting

$$
g_{k}=\left(f_{k}-\pi_{k} f_{k}\right)\left\|f_{k}-\pi_{k} f_{k}\right\|_{L^{2}(0, T)}^{-2}, \quad \forall k \in \mathbb{Z}^{*}
$$

where $\pi_{k}$ is the orthogonal projector onto $\overline{\operatorname{span}\left\{f_{j}: j \neq k\right\}} L^{2}$. The unicity of $\left(g_{k}\right)_{k \in \mathbb{Z}^{*}}$ follows as, for any biorthogonal family $\left(g_{k}^{1}\right)_{k \in \mathbb{Z}^{*}}$ in $X$, we have $\left\langle g_{k}-g_{k}^{1}, f_{j}\right\rangle_{L^{2}(0, T)}=0$ for every $j, k \in \mathbb{Z}^{*}$, which implies $g_{k}=g_{k}^{1}$ for every $k \in \mathbb{Z}^{*}$.

Remark 3.6. If a sequence of functions $\left(f_{k}\right)_{k \in \mathbb{Z}^{*}} \subset L^{2}((0, T), \mathbb{C})$ admits a biorthogonal family $\left(g_{k}\right)_{k \in \mathbb{Z}^{*}}$, then it is minimal. Indeed, if we assume that there exists $k \in \mathbb{Z}^{*}$ such that $f_{k} \in \overline{\operatorname{span}\left\{f_{j}: j \neq k\right\}} L^{2}$, then the relations $\left\langle f_{k}, g_{j}\right\rangle_{L^{2}(0, T)}=0$ for every $j \in \mathbb{Z}^{*} \backslash\{k\}$ would imply $\left\langle f_{k}, g_{k}\right\rangle_{L^{2}(0, T)}=0$ which is absurd.

Definition 3.7. Let $\left(f_{k}\right)_{k \in \mathbb{Z}^{*}}$ be a family of functions in $L^{2}((0, T), \mathbb{C})$ with $T>0$. The family $\left(f_{k}\right)_{k \in \mathbb{Z}^{*}}$ is a Riesz basis of $\overline{\operatorname{span}\left\{f_{j}: j \in \mathbb{Z}^{*}\right\}} L^{2}$ if and only if it is isomorphic to an orthonormal system.

Remark 3.8. Let $\left(f_{k}\right)_{k \in \mathbb{Z}^{*}}$ be a Riesz basis of $X:=\overline{\operatorname{span}\left\{f_{j}: j \in \mathbb{Z}^{*}\right\}}{ }^{2}$. The sequence $\left(f_{k}\right)_{k \in \mathbb{Z}^{*}}$ is also minimal and the biorthogonal family to $\left(f_{k}\right)_{k \in \mathbb{Z}^{*}}$ can be uniquely defined in $X$ thanks to Remark 3.5. The biorthogonal family to $\left(f_{k}\right)_{k \in \mathbb{Z}^{*}}$ forms a Riesz basis of $X$.

Now, we provide an important property on the Riesz basis proved in [BL10, Appendix B.1].

Proposition 3.9. [BL10, Appendix B; Proposition 19] Let $\left(f_{k}\right)_{k \in \mathbb{Z}^{*}}$ be a family of functions in $L^{2}((0, T), \mathbb{C})$ with $T>0$ and let $\left(f_{k}\right)_{k \in \mathbb{Z}^{*}}$ be a Riesz basis of $\overline{\operatorname{span}\left\{f_{k}: k \in \mathbb{Z}^{*}\right\}}{ }^{2}$. There exist $C_{1}, C_{1}>0$ such that

$$
C_{1}\|\mathbf{x}\|_{\ell^{2}}^{2} \leq \int_{0}^{T}\left|\sum_{k \in \mathbb{Z}^{*}} x_{k} f_{k}\right|^{2} d s \leq C_{2}\|\mathbf{x}\|_{\ell^{2}}^{2}, \quad \forall \mathbf{x}:=\left(x_{k}\right)_{k \in N^{*}} \in \ell^{2}\left(\mathbb{Z}^{*}, \mathbb{C}\right) .
$$

\subsection{Riesz basis made by divided differences of exponentials}

Let $\boldsymbol{v}=\left(\nu_{k}\right)_{k \in \mathbb{Z}^{*}} \subset \mathbb{R}$ be an ordered sequence of pairwise distinct numbers such that there exist $\mathcal{M} \in \mathbb{N}^{*}$ and $\delta>0$ such that

$$
\inf _{\left\{k \in \mathbb{Z}^{*}: k+\mathcal{M} \neq 0\right\}}\left|\nu_{k+\mathcal{M}}-\nu_{k}\right| \geq \delta \mathcal{M}
$$


The last relation yields that it does not exist $\mathcal{M}$ consecutive $k, k+1 \in \mathbb{Z}^{*}$ such that $\left|\nu_{k+1}-\nu_{k}\right|<\delta$ and then, there exist some $j \in \mathbb{Z}^{*} \backslash\{-1\}$ such that $\left|\nu_{j+1}-\nu_{j}\right| \geq \delta$. This fact leads to a partition of $\mathbb{Z}^{*}$ in subsets $\left\{E_{m}\right\}_{m \in \mathbb{Z}^{*}}$ that we construct as follows. We denote by $\left(l_{m}\right)_{m \in \mathbb{Z}^{*}} \subseteq \mathbb{Z}^{*} \backslash\{-1\}$ the ordered sequence of all the numbers such that

$$
\left|\nu_{l_{m}+1}-\nu_{l_{m}}\right| \geq \delta \text {. }
$$

We also add the value -1 to such sequence when $\left|\nu_{1}-\nu_{-1}\right| \geq \delta$. We denote by $\left\{E_{m}\right\}_{m \in \mathbb{Z}^{*}}$ the sets:

$$
\begin{gathered}
E_{-1}=\left\{k \in \mathbb{Z}^{*}: l_{-1}+1 \leq k \leq l_{1}\right\}, \\
E_{m}=\left\{k \in \mathbb{Z}^{*}: l_{m}+1 \leq k \leq l_{m+1}\right\}, \quad \forall m \in \mathbb{Z}^{*} \backslash\{-1\} .
\end{gathered}
$$

The partition of $\mathbb{Z}^{*}$ in subsets $\left\{E_{m}\right\}_{m \in \mathbb{Z}^{*}}$ also defines an equivalence relation in $\mathbb{Z}^{*}$ :

$$
k \sim n \text { if and only if there exists } m \in \mathbb{Z}^{*} \text { such that } k, n \in E_{m}
$$

Now, $\left\{E_{m}\right\}_{m \in \mathbb{Z}^{*}}$ are the equivalence classes corresponding to such relation and $\left|E_{m}\right| \leq \mathcal{M}-1$ thanks to (11). Let $s(m)$ be the smallest element of $E_{m}$. For every $\mathbf{x}:=\left(x_{k}\right)_{k \in \mathbb{Z}^{*}} \subset \mathbb{C}$ and $m \in \mathbb{Z}^{*}$, we define

$$
\mathbf{x}^{m}:=\left(x_{l}^{m}\right)_{l \leq\left|E_{m}\right|}, \quad: \quad x_{l}^{m}=x_{s(m)+(l-1)}, \quad \forall l \leq\left|E_{m}\right| .
$$

In other words, $\mathbf{x}^{m}$ is the vector in $\mathbb{C}^{\left|E_{m}\right|}$ composed by those elements of $\mathbf{x}$ with indices in $E_{m}$. For every $m \in \mathbb{Z}^{*}$, we denote $F_{m}\left(v^{m}\right): \mathbb{C}^{\left|E_{m}\right|} \rightarrow \mathbb{C}^{\left|E_{m}\right|}$ the matrix with components

$$
F_{m ; j, k}\left(v^{m}\right):=\left\{\begin{array}{ll}
\prod_{\substack{l \neq j \\
l \leq k}}\left(\nu_{j}^{m}-\nu_{l}^{m}\right)^{-1}, & j \leq k, \\
1, & j=k=1, \\
0, & j>k,
\end{array} \quad \forall j, k \leq\left|E_{m}\right| .\right.
$$

For each $k \in \mathbb{Z}^{*}$, there exists $m(k) \in \mathbb{Z}^{*}$ such that $k \in E_{m(k)}$, while $s(m(k))$ represents the smallest element of $E_{m(k)}$. Let $F(v)$ be the infinite matrix acting on $\mathbf{x}=\left(x_{k}\right)_{k \in \mathbb{Z}^{*}} \subset \mathbb{C}$ as follows

$$
(F(v) \mathbf{x})_{k}=\left(F_{m(k)}\left(v^{m(k)}\right) \mathbf{x}^{m(k)}\right)_{k-s(m(k))+1}, \quad \forall k \in \mathbb{Z}^{*}
$$

We consider $F(v)$ as the operator on $\ell^{2}\left(\mathbb{Z}^{*}, \mathbb{C}\right)$ defined by the action above and with domain

$$
H(v):=D(F(v))=\left\{\mathbf{x}:=\left(x_{k}\right)_{k \in \mathbb{Z}^{*}} \in \ell^{2}\left(\mathbb{Z}^{*}, \mathbb{C}\right): F(v) \mathbf{x} \in \ell^{2}\left(\mathbb{Z}^{*}, \mathbb{C}\right)\right\}
$$

Remark 3.10. Each matrix $F_{m}\left(v^{m}\right)$ with $m \in \mathbb{Z}^{*}$ is invertible and we call $F_{m}\left(v^{m}\right)^{-1}$ its inverse. Now, $F(v): H(v) \rightarrow \operatorname{Ran}(F(v))$ is invertible and $F(v)^{-1}: \operatorname{Ran}(F(v)) \rightarrow H(v)$ is so that, for $\mathbf{x} \in \operatorname{Ran}(F(v))$,

$$
\left(F(v)^{-1} \mathbf{x}\right)_{k}=\left(F_{m(k)}\left(v^{m(k)}\right)^{-1} \mathbf{x}^{m(k)}\right)_{k-s(m(k))+1}, \quad \forall k \in \mathbb{Z}^{*}
$$

Let $F_{m(k)}\left(v^{m(k)}\right)^{*}$ be the transposed matrix of $F_{m(k)}\left(v^{m(k)}\right)$ for every $m \in \mathbb{Z}^{*}$. Let $F(v)^{*}$ be the infinite matrix so that, for every $\mathbf{x}=\left(x_{k}\right)_{k \in \mathbb{Z}^{*}} \subset \mathbb{C}$,

$$
\left(F(v)^{*} \mathbf{x}\right)_{k}=\left(F_{m(k)}\left(v^{m(k)}\right)^{*} \mathbf{x}^{m(k)}\right)_{k-s(m(k))+1}, \quad \forall k \in \mathbb{Z}^{*}
$$

Remark 3.11. When $H(v)$ is dense in $\ell^{2}\left(\mathbb{Z}^{*}, \mathbb{C}\right)$, we can consider $F(v)^{*}$ as the unique adjoint operator of $F(v)$ in $\ell^{2}\left(\mathbb{Z}^{*}, \mathbb{C}\right)$ with domain $H(v)^{*}:=D\left(F(v)^{*}\right)$. As in Remark 3.10, we define $\left(F(v)^{*}\right)^{-1}$ the inverse operator of $F(v)^{*}: H(v)^{*} \rightarrow \operatorname{Ran}\left(F(v)^{*}\right)$ and $\left(F(v)^{*}\right)^{-1}=\left(F(v)^{-1}\right)^{*}$.

Let $\mathbf{e}$ be the sequence of functions in $L^{2}((0, T), \mathbb{C})$ with $T>0$ so that $\mathbf{e}:=\left(e^{i \nu_{k}(\cdot)}\right)_{k \in \mathbb{Z}^{*}}$. We denote by $\boldsymbol{\Xi}$ the so-called divided differences of the family $\left(e^{i \nu_{k} t}\right)_{k \in \mathbb{Z}^{*}}$ such that

$$
\boldsymbol{\Xi}:=\left(\xi_{k}\right)_{k \in \mathbb{Z}^{*}}=F(v)^{*} \mathbf{e}
$$

In the following theorem, we rephrase a result of Avdonin and Moran [AM01], which is also proved by Baiocchi, Komornik and Loreti in [BKL02]. 
Theorem 3.12 (Theorem 3.29; [DZ06]). Let $\left(\nu_{k}\right)_{k \in \mathbb{Z}^{*}}$ be an ordered sequence of pairwise distinct real numbers satisfying (11). If $T>2 \pi / \delta$, then $\left(\xi_{k}\right)_{k \in \mathbb{Z}^{*}}$ forms a Riesz Basis in the space $\overline{\operatorname{span}\left\{\xi_{k}: k \in \mathbb{Z}^{*}\right\}} L^{2}$.

Remark 3.13. Let Theorem 3.12 be valid. As $\boldsymbol{\Xi}$ is a Riesz basis, it is minimal in ${\overline{\operatorname{span}\left\{\xi_{k}: k \in \mathbb{Z}^{*}\right\}}}^{L^{2}}$ and it admits a biorthogonal family $\mathbf{u}:=\left(u_{k}\right)_{k \in \mathbb{Z}^{*}}$ thanks to Remark 3.6. Now, it is possible to see that

$$
\left\langle u_{k}, \xi_{j}\right\rangle_{L^{2}(0, T)}=\left\langle u_{k},\left(F(\boldsymbol{v})^{*} \mathbf{e}\right)_{j}\right\rangle_{L^{2}(0, T)}=\left\langle(F(v) \mathbf{u})_{k}, e^{i \nu_{j} t}\right\rangle_{L^{2}(0, T)}, \quad \forall j, k \in \mathbb{Z}^{*} .
$$

The last relation ensures that $F(v) \mathbf{u}$ is a biorthogonal family to $\mathbf{e}$, which is then minimal.

\subsection{Auxiliary results}

In this subsection, we provide few intermediate results required in the proof of Proposition 3.1.

In Lemma 3.14 and Lemma 3.15, we consider a suitable sequence of numbers $v:=\left(\nu_{k}\right)_{k \in \mathbb{Z}^{*}}$. We construct and characterize a biorthogonal family to $\left\{e^{i \nu_{k} t}\right\}_{k \in \mathbb{Z}^{*}}$ by using a function $G$ defined as in Proposition 3.1. In Lemma 3.16, the previous results lead to specific estimations on $F_{m}\left(v^{m}\right)$ with $m \in \mathbb{Z}^{*}$.

In Lemma 3.18 and Lemma 3.19, we consider a sequence $\boldsymbol{\Theta}:=\left(\theta_{k}\right)_{k \in \mathbb{Z}^{*}}$ and another one defined as $\checkmark:=\left(\nu_{k}\right)_{k \in \mathbb{Z}^{*}}=\left(\operatorname{sgn}\left(\theta_{k}\right) \sqrt{\left|\theta_{k}\right|}\right)_{k \in \mathbb{Z}^{*}}$. We use the estimations on $F_{m}\left(\nu^{m}\right)$ with $m \in \mathbb{Z}^{*}$ provided by Lemma 3.16 in order to study $F_{m}\left(\mathbf{\Theta}^{m}\right)$ with $m \in \mathbb{Z}^{*}$ and on the domain of the operator $F(\boldsymbol{\Theta})$.

In the proof of Proposition 3.1, we respectively denote by $\Theta$ and $v$ the sequences obtained by reordering $\left\{ \pm \lambda_{k}\right\}_{k \in \mathbb{N}^{*}}$ and $\left\{ \pm \sqrt{\lambda_{k}}\right\}_{k \in \mathbb{N}^{*}}$ and we use Lemma 3.19 to ensure the statement.

Lemma 3.14. Let $v:=\left(\nu_{k}\right)_{k \in \mathbb{Z}^{*}}$ be an ordered sequence of pairwise distinct real numbers. Let $G$ be an entire function such that $G \in L^{\infty}(\mathbb{R}, \mathbb{R})$. Let exist $J, I>0$ such that $|G(z)| \leq J e^{I|z|}$ for every $z \in \mathbb{C}$. Denoted $G_{k}(z):=G(z)\left(z-\nu_{k}\right)^{-1}$ with $z \in \mathbb{C}$ for every $k \in \mathbb{Z}^{*}$, there exists $C>0$ such that

$$
\left\|G_{k}\right\|_{L^{2}(\mathbb{R}, \mathbb{R})} \leq C, \quad \forall k \in \mathbb{Z}^{*} .
$$

Proof. We know that there exists $M>0$ so that $|G(x)| \leq M$ for every $x \in \mathbb{R}$. It implies that, for every $k \in \mathbb{Z}^{*}$, there exists $C_{1}>0$ (not depending on $k$ ) so that

$$
\left\|G_{k}\right\|_{L^{2}(\mathbb{R})}^{2}=\int_{\mathbb{R}} \overline{G_{k}(x)} G_{k}(x) d x=\int_{\mathbb{R}} \frac{\overline{G(x)} G(x)}{\left(x-\nu_{k}\right)^{2}} d x \leq \int_{\left|x-\nu_{k}\right| \leq 1} \overline{G(x)} G(x)\left(x-\nu_{k}\right)^{-2} d x+M^{2} C_{1} .
$$

Now, from [You80,p. 82; Theorem 11], we have $|G(x+i y)| \leq M e^{I|y|}$ for $x, y \in \mathbb{R}$. The Cauchy Integral Theorem ensures the existence of $C_{2}>0$ (not depending on $k$ ) so that

$$
\int_{\left|x-\nu_{k}\right| \leq 1} \frac{\overline{G(x)} G(x)}{\left(x-\nu_{k}\right)^{2}} d x \leq \int_{0}^{\pi} \overline{\mid \overline{\left(\overline{\nu_{k}+e^{i \theta}}\right)}} G\left(\nu_{k}+e^{i \theta}\right) \mid d \theta \leq M^{2} \int_{0}^{\pi} e^{2 I \sin (\theta)} d \theta \leq M^{2} C_{2} .
$$

Lemma 3.15. Let $v:=\left(\nu_{k}\right)_{k \in \mathbb{Z}^{*}}$ be an ordered sequence of pairwise distinct real numbers satisfying (11) with $\delta>0$. Let $G$ be an entire function such that $G \in L^{\infty}(\mathbb{R}, \mathbb{R})$. Let exist $J, I>0$ such that $|G(z)| \leq J e^{I|z|}$ for every $z \in \mathbb{C}$. Let $\left(\xi_{k}\right)_{k \in \mathbb{Z}^{*}} \subset L^{2}((0, T), \mathbb{C})$ be defined in $(13)$ for $T>\max \{2 \pi / \delta, 2 I\}$. If $\left(\nu_{k}\right)_{k \in \mathbb{Z}^{*}}$ are simple zeros of $G$ such that there exist $\tilde{d} \geq 0, C>0$ such that

$$
\left|G^{\prime}\left(\nu_{k}\right)\right| \geq \frac{C}{|k|^{1+\tilde{d}}}, \quad \forall k \in \mathbb{Z}^{*}
$$

then there exists $\left(w_{k}\right)_{k \in \mathbb{Z}^{*}}$ an unique biorthogonal family to $\left(e^{i \nu_{k} t}\right)_{k \in \mathbb{Z}^{*}}$ in $\overline{\operatorname{span}\left\{\xi_{k}: k \in \mathbb{Z}^{*}\right\}} L^{2}$ satisfying the following property. There exists $C_{1}>0$ such that $\left\|w_{k}\right\|_{L^{2}(0, T)} \leq C_{1}|k|^{1+\tilde{d}}$ for every $k \in \mathbb{Z}^{*}$.

Proof. For every $k \in \mathbb{Z}^{*}$, we define $G_{k}(z):=G(z)\left(z-\nu_{k}\right)^{-1}$. Thanks to the Paley-Wiener's Theorem [DZ06, Theorem 3.19], for every $k \in \mathbb{Z}^{*}$, there exists $f_{k} \in L^{2}(\mathbb{R}, \mathbb{R})$ with support in $[-I, I]$ such that

$$
G_{k}(z)=\int_{-I}^{I} e^{i z s} f_{k}(s) d s=\int_{-T / 2}^{T / 2} e^{i z s} f_{k}(s) d s=\int_{0}^{T} e^{i z t} e^{-i z \frac{T}{2}} f_{k}(t-T / 2) d t .
$$

For $j, k \in \mathbb{Z}^{*}$ and $c_{k}:=G^{\prime}\left(\nu_{k}\right)$, we name $v_{k}(t):=e^{i \nu_{k} \frac{T}{2}} \overline{f_{k}}(t-T / 2)$. Since $G_{k}\left(\nu_{j}\right)=\delta_{k, j} G^{\prime}\left(\nu_{k}\right)$,

$$
\left\langle v_{k}, e^{i \nu_{j}(\cdot)}\right\rangle_{L^{2}(0, T)}=G_{k}\left(\nu_{j}\right)=\delta_{k, j} G^{\prime}\left(\nu_{k}\right)=\delta_{k, j} c_{k}
$$


Thus, the sequence $\left(v_{k}\right)_{k \in \mathbb{Z}^{*}}$ is biorthogonal to $\left(c_{k}^{-1} e^{i \nu_{k}(\cdot)}\right)_{k \in \mathbb{Z}^{*}}$. Thanks to the Plancherel's identity and to Lemma 3.14, there exists $C_{1}>0$ such that

$$
\left\|v_{k}\right\|_{L^{2}(0, T)}=\left\|G_{k}\right\|_{L^{2}(\mathbb{R}, \mathbb{R})} \leq C_{1}, \quad \forall k \in \mathbb{Z}^{*} .
$$

The family $\left(e^{i \nu_{k}(\cdot)}\right)_{k \in \mathbb{Z}^{*}}$ is minimal in $X:=\overline{\operatorname{span}\left\{\xi_{k}: k \in \mathbb{Z}^{*}\right\}} L^{2}$ thanks to Remark 3.13 and then, $\left(c_{k}^{-1} e^{i \nu_{k}(\cdot)}\right)_{k \in \mathbb{Z}^{*}}$ is minimal in $X$. Defined $\pi_{X}$ the orthogonal projector onto $X$, we see that, for every $j, k \in \mathbb{Z}^{*}$,

$$
\left\langle\pi_{X} v_{k}, c_{j}^{-1} e^{i \nu_{j}(\cdot)}\right\rangle_{L^{2}(0, T)}=\left\langle v_{k}, c_{j}^{-1} e^{i \nu_{j}(\cdot)}\right\rangle_{L^{2}(0, T)}=\delta_{k, j} .
$$

The last relation and Remark 3.5 imply that $\left(\pi_{X} v_{k}\right)_{k \in \mathbb{Z}^{*}}$ is the unique biorthogonal family to $\left(c_{k}^{-1} e^{i \nu_{k}(\cdot)}\right)_{k \in \mathbb{Z}^{*}}$ in $X$. We denote $w_{k}=\left(\overline{c_{k}}\right)^{-1} \pi_{X} v_{k}$ for every $k \in \mathbb{Z}^{*}$ and $\left(w_{k}\right)_{k \in \mathbb{Z}^{*}}$ is the unique biorthogonal family to $\left(e^{i \nu_{k}(\cdot)}\right)_{k \in \mathbb{Z}^{*}}$ in $X$. In conclusion, thanks to (14) and (15), there exists $C_{2}>0$ such that

$$
\left\|w_{k}\right\|_{L^{2}(0, T)} \leq\left\|\pi_{X} v_{k}\right\|_{L^{2}(0, T)}\left|c_{k}\right|^{-1} \leq\left\|v_{k}\right\|_{L^{2}(0, T)}\left|G^{\prime}\left(\nu_{k}\right)\right|^{-1} \leq C_{2}|k|^{1+\tilde{d}}, \quad \forall k \in \mathbb{Z}^{*} .
$$

Lemma 3.16. Let $v:=\left(\nu_{k}\right)_{k \in \mathbb{Z}^{*}}$ be an ordered sequence of pairwise distinct real numbers satisfying (11). Let $G$ be an entire function such that $G \in L^{\infty}(\mathbb{R}, \mathbb{R})$. Let exist $J, I>0$ such that $|G(z)| \leq J e^{I|z|}$ for every $z \in \mathbb{C}$. If $\left(\nu_{k}\right)_{k \in \mathbb{Z}^{*}}$ are simple zeros of $G$ such that there exist $\tilde{d} \geq 0, C>0$ such that $\left|G^{\prime}\left(\nu_{k}\right)\right| \geq \frac{C}{|k|^{1+d}}$ for every $k \in \mathbb{Z}^{*}$, then there exists $C>0$ so that

$$
\operatorname{Tr}\left(F_{m}\left(\nu^{m}\right)^{*} F_{m}\left(v^{m}\right)\right) \leq C \min \left\{|l| \in E_{m}\right\}^{2(1+\tilde{d})}, \quad \forall m \in \mathbb{Z}^{*}
$$

where the matrices $F_{m}\left(v^{m}\right)$ are defined in Section 3.3.

Proof. Let $T>\max (2 \pi / \delta, 2 I)$ with $\delta>0$ from (11). Thanks to Theorem 3.12, the sequence of functions $\boldsymbol{\Xi}=\left(\xi_{k}\right)_{k \in \mathbb{Z}^{*}}:=\left(F(v)^{*} \mathbf{e}\right)$ forms a Riesz basis of $X:=\overline{\operatorname{span}\left\{\xi_{k}: k \in \mathbb{Z}^{*}\right\}}{ }^{2}$. We call $\mathbf{u}:=\left(u_{k}\right)_{k \in \mathbb{Z}^{*}}$ the corresponding biorthogonal sequence to $\boldsymbol{\Xi}$ in $X$, which is also a Riesz basis of $X$ (see Remark 3.8). From Remark 3.10, the matrix $F(v)$ is invertible and $\left(F(v)^{*}\right)^{-1}=\left(F(v)^{-1}\right)^{*}$ thanks to Remark 3.11. Let $\mathbf{w}=\left(w_{k}\right)_{k \in \mathbb{Z}^{*}}$ be the biorthogonal family in $X$ to $\left(e^{i \nu_{k}(\cdot)}\right)_{k \in \mathbb{Z}^{*}}$ defined in Lemma 3.15. For every $j, k \in \mathbb{Z}^{*}$,

$$
\delta_{k, j}=\left\langle w_{k}, e^{i \nu_{j}(\cdot)}\right\rangle_{L^{2}(0, T)}=\left\langle w_{k},\left(\left(F(v)^{*}\right)^{-1} \boldsymbol{\Xi}\right)_{j}\right\rangle_{L^{2}(0, T)}=\left\langle\left(F(v)^{-1} \mathbf{w}\right)_{k}, \xi_{j}\right\rangle_{L^{2}(0, T)},
$$

implying $\mathbf{w}=F(v) \mathbf{u}$. Let $f(t)=\sum_{k \in \mathbb{Z}^{*}} \xi_{k} x_{k}$ with $\mathbf{x}:=\left(x_{k}\right)_{k \in \mathbb{Z}^{*}} \in \ell^{2}\left(\mathbb{Z}^{*}, \mathbb{C}\right)$. As $\boldsymbol{\Xi}$ and $\mathbf{u}$ are reciprocally biorthogonal, there holds $x_{k}=\left\langle u_{k}, f\right\rangle_{L^{2}(0, T)}$ for every $k \in \mathbb{Z}^{*}$. From Proposition 3.9, there exist $C_{1}, C_{2}>0$ such that

$$
C_{1}\|\mathbf{x}\|_{\ell^{2}}^{2} \leq\|f\|_{L^{2}(0, T)}^{2} \leq C_{2}\|\mathbf{x}\|_{\ell^{2}}^{2} .
$$

For $k \in \mathbb{Z}^{*}$, we call $m(k) \in \mathbb{Z}^{*}$ the number such that $k \in E_{m(k)}$. Thanks to Lemma 3.15 and (16), there exist $C_{3}, C_{4}>0$ such that, for every $k \in \mathbb{Z}^{*}$, we have

$$
\begin{aligned}
\left|(F(v) \mathbf{x})_{k}\right| & =\left|\left(F(v)\left(\left\langle u_{l}, f\right\rangle_{L^{2}(0, T)}\right)_{l \in \mathbb{Z}^{*}}\right)_{k}\right|=\left|\left\langle w_{k}, f\right\rangle_{L^{2}(0, T)}\right| \leq\left\|w_{k}\right\|_{L^{2}(0, T)}\|f\|_{L^{2}(0, T)} \\
& \leq C_{2}^{\frac{1}{2}}\left\|w_{k}\right\|_{L^{2}(0, T)}\|\mathbf{x}\|_{\ell^{2}} \leq C_{3}|k|^{1+\widetilde{d}}\|\mathbf{x}\|_{\ell^{2}} \leq C_{4} \min _{l \in E_{m(k)}}|l|^{1+\widetilde{d}}\|\mathbf{x}\|_{\ell^{2}} .
\end{aligned}
$$

For every $m \in \mathbb{Z}^{*}$, we denote by $s(m)$ the smallest element of $E_{m}$ and, for every $j \leq\left|E_{m}\right|$, we consider

$$
\mathbf{x} \in \ell^{2}\left(\mathbb{Z}^{*}, \mathbb{C}\right) \quad: \quad x_{s(m)+j-1}=1, \quad x_{l}=0, \quad \forall l \in \mathbb{Z}^{*} \backslash\{s(m)+j-1\} .
$$

For every $j, n \leq\left|E_{m}\right|$, we use the sequence $\mathbf{x}$ in the identity (17) with $k=s(m)+n-1$ and we obtain $\left|\left(F_{m ; n, j}\left(\nu^{m}\right)\right)\right| \leq C_{4} \min _{l \in E_{m}}|l|^{1+\widetilde{d}}$, which leads to the statement.

Remark 3.17. Let $\Theta:=\left(\theta_{k}\right)_{k \in \mathbb{Z}^{*}}$ be an ordered sequence of pairwise distinct real numbers and let the sequence $\vee:=\left(\nu_{k}\right)_{k \in \mathbb{Z}^{*}}=\left(\operatorname{sgn}\left(\theta_{k}\right) \sqrt{\left|\theta_{k}\right|}\right)_{k \in \mathbb{Z}^{*}}$ satisfy the identity (11) with $\mathcal{M} \in \mathbb{N}^{*}$ and $\delta>0$. As $\inf _{\substack{k \in \mathbb{Z}^{*} \\ k+\mathcal{M} \neq 0}}\left|\nu_{k+\mathcal{M}}-\nu_{k}\right| \geq \delta \mathcal{M} \min _{\substack{k \in \mathbb{Z}^{*} \\ \nu_{k} \neq 0}}\left(\left|\nu_{k}\right|, 1\right)$, we have

$$
\inf _{\substack{k \in \mathbb{Z}^{*} \\ k+\mathcal{M} \neq 0}}\left|\theta_{k+\mathcal{M}}-\theta_{k}\right|=\inf _{\substack{k \in \mathbb{Z}^{*} \\ k+\mathcal{M} \neq 0}}|| \nu_{k+\mathcal{M}}|-| \nu_{k}|||| \nu_{k+\mathcal{M}}|+| \nu_{k}|| \geq \min _{\substack{k \in \mathbb{Z}^{*} \\ \nu_{k} \neq 0}}\left(\left|\nu_{k}\right|, 1\right) \delta \mathcal{M} .
$$

Now, both the sequences $\Theta$ and $\vee$ satisfy (11) with respect to the same $\delta^{\prime}:=\min _{\substack{k \in \mathbb{Z}^{*} \\ \nu_{k} \neq 0}}\left\{\left|\nu_{k}\right|, 1\right\} \delta$ and $\mathcal{M}$. This fact ensures that $\Theta$ and $\vee$ induce the definition of the same equivalence classes $\left\{E_{m}\right\}_{m \in \mathbb{Z}^{*}}$ of $\mathbb{Z}^{*}$ introduced in the relations (12). Thus, we can use $\left\{E_{m}\right\}_{m \in \mathbb{Z}^{*}}$ in order to define the matrices $F_{m}\left(\Theta^{m}\right)$ and $F_{m}\left(\vee^{m}\right)$ for every $m \in \mathbb{Z}^{*}$ and the operators $F(\Theta)$ and $F(v)$ on $\ell^{2}\left(\mathbb{Z}^{*}, \mathbb{C}\right)$. 
Lemma 3.18. Let $\boldsymbol{\Theta}:=\left(\theta_{k}\right)_{k \in \mathbb{Z}^{*}}$ be an ordered sequence of pairwise distinct real numbers such that $\left(\nu_{k}\right)_{k \in \mathbb{Z}^{*}}=\left(\operatorname{sgn}\left(\theta_{k}\right) \sqrt{\left|\theta_{k}\right|}\right)_{k \in \mathbb{Z}^{*}}$ satisfies (11). Let exist $C_{1}, C_{2}>0$ such that

$$
C_{1}|k| \leq\left|\nu_{k}\right| \leq C_{2}|k|, \quad \forall k \in \mathbb{Z}^{*}, \quad \nu_{k} \neq 0 .
$$

Let $G$ be an entire function so that $\left(\nu_{k}\right)_{k \in \mathbb{Z}^{*}}$ are its simple zeros, $G \in L^{\infty}(\mathbb{R}, \mathbb{R})$ and there exist $J, I>0$ such that $|G(z)| \leq J e^{I|z|}$ for every $z \in \mathbb{C}$. If there exist $\tilde{d} \geq 0$ and $C>0$ such that $\left|G^{\prime}\left(\nu_{k}\right)\right| \geq \frac{C}{|k|^{1+\tilde{d}}}$ for every $k \in \mathbb{Z}^{*}$, then there exists $C>0$ so that

$$
\operatorname{Tr}\left(F_{m}\left(\boldsymbol{\Theta}^{m}\right)^{*} F_{m}\left(\boldsymbol{\Theta}^{m}\right)\right) \leq C \min \left\{|l| \in E_{m}\right\}^{2 \tilde{d}}, \quad \forall m \in \mathbb{Z}^{*}
$$

where the matrices $F_{m}\left(\boldsymbol{\Theta}^{m}\right)$ are defined as in Remark 3.17 from the sequence $\boldsymbol{\Theta}$.

Proof. We notice $\left|\theta_{l}-\theta_{k}\right| \geq \min \left\{\left|\nu_{l}\right|,\left|\nu_{k}\right|\right\}\left|\nu_{l}-\nu_{k}\right|$ for every $l, k \in \mathbb{Z}^{*}$. Let $m \in \mathbb{Z}^{*}$ and $I \subseteq E_{m}$ so that $I \neq \emptyset$. Now, $|I| \leq\left|E_{m}\right| \leq \mathcal{M}-1$ and

$$
\prod_{j, k \in I}\left|\theta_{k}-\theta_{j}\right| \geq \min _{\substack{l \in I \\ \nu_{l} \neq 0}}\left|\nu_{l}\right|^{|I|} \prod_{j, k \in I}\left|\nu_{k}-\nu_{j}\right| \geq C_{1} \min _{\substack{l \in I \\ \nu_{l} \neq 0}}\left|\nu_{l}\right| \prod_{j, k \in I}\left|\nu_{k}-\nu_{j}\right|
$$

for $C_{1}=\min _{\substack{l \in \mathbb{Z}^{*} \\ \nu_{l} \neq 0}}\left(\left|\nu_{l}\right|^{\mathcal{M}-2}, 1\right)$. Thus, there exists $C_{2}>0$ so that, for every $m$ and $j, k \in E_{m}$, we have

$$
\left|F_{m ; j, k}\left(\boldsymbol{\Theta}^{m}\right)\right| \leq C_{2}\left|F_{m ; j, k}\left(\nu^{m}\right)\right| \min \left\{\left|\nu_{l}\right|^{-1}: l \in E_{m}, \nu_{l} \neq 0\right\} .
$$

In conclusion, thanks to (18) and Lemma 3.16, there exists $C_{3}>0$ such that

$$
\operatorname{Tr}\left(F_{m}\left(\Theta^{m}\right)^{*} F_{m}\left(\Theta^{m}\right)\right) \leq C_{2}^{2} \min _{\substack{l \in E_{m} \\ \nu_{l} \neq 0}}\left|\nu_{l}\right|^{-2} \operatorname{Tr}\left(F_{m}\left(\nu^{m}\right)^{*} F_{m}\left(\nu^{m}\right)\right) \leq C_{3} \min _{l \in E_{m}}|l|^{2 \tilde{d}} .
$$

Lemma 3.19. Let the hypotheses of Lemma 3.18 be verified. Then, there holds $H(\Theta) \subseteq h^{\tilde{d}}\left(\mathbb{Z}^{*}, \mathbb{C}\right)$ where $H(\boldsymbol{\Theta})$ is the domain of the operator $F(\boldsymbol{\Theta})$ defined as in Remark 3.17 from the sequence $\boldsymbol{\Theta}$.

Proof. Let $\rho(M)$ be the spectral radius of a matrix $\mathrm{M}$ and let $\|M\|=\sqrt{\rho\left(M^{*} M\right)}$ be its euclidean norm. We consider Lemma 3.18 and, since $\left(F_{m}\left(\boldsymbol{\Theta}^{m}\right)^{*} F_{m}\left(\mathbf{\Theta}^{m}\right)\right)$ is positive-definite, there exists $C>0$ such that

$$
\left\|\left.\left|F_{m}\left(\boldsymbol{\Theta}^{m}\right) \|^{2}=\rho\left(F_{m}\left(\Theta^{m}\right)^{*} F_{m}\left(\Theta^{m}\right)\right) \leq \operatorname{Tr}\left(F_{m}\left(\Theta^{m}\right)^{*} F_{m}\left(\Theta^{m}\right)\right) \leq C \min _{l \in E_{m}}\right| l\right|^{2 \tilde{d}}, \quad m \in \mathbb{Z}^{*} .\right.
$$

In conclusion, we obtain $h^{\tilde{d}}\left(\mathbb{Z}^{*}, \mathbb{C}\right) \subset H(\boldsymbol{\Theta})$ since, for every $\mathbf{x}=\left(x_{k}\right)_{k \in \mathbb{Z}^{*}} \in h^{\tilde{d}}\left(\mathbb{Z}^{*}, \mathbb{C}\right)$,

$$
\|F(\boldsymbol{\Theta}) \mathbf{x}\|_{\ell^{2}}^{2} \leq \sum_{m \in \mathbb{Z}^{*}}\left\|\left|F_{m}\left(\boldsymbol{\Theta}^{m}\right)\left\|\left.\right|^{2} \sum_{l \in E_{m}}\left|x_{l}\right|^{2} \leq C \sum_{m \in \mathbb{Z}^{*}} \min _{l \in E_{m}}|l|^{2 \tilde{d}} \sum_{l \in E_{m}}\left|x_{l}\right|^{2} \leq C\right\| \mathbf{x} \|_{h^{\tilde{d}}}^{2}<+\infty .\right.\right.
$$

Remark 3.20. When Lemma 3.19 is satisfied with respect to the sequence $\boldsymbol{\Theta}=\left(\theta_{k}\right)_{k \in \mathbb{Z}^{*}}$ with $\tilde{d} \geq 0$, we have $H(\boldsymbol{\Theta}) \supseteq h^{\tilde{d}}\left(\mathbb{Z}^{*}, \mathbb{C}\right)$ that is dense in $\ell^{2}\left(\mathbb{Z}^{*}, \mathbb{C}\right)$. Thanks to Remark 3.11 , we consider $F(\boldsymbol{\Theta})^{*}$ as the unique adjoint operator of $F(\boldsymbol{\Theta})$. As $\operatorname{Tr}\left(F_{m}\left(\boldsymbol{\Theta}^{m}\right)^{*} F_{m}\left(\mathbf{\Theta}^{m}\right)\right)=\operatorname{Tr}\left(F_{m}\left(\mathbf{\Theta}^{m}\right) F_{m}\left(\mathbf{\Theta}^{m}\right)^{*}\right)$ for every $m \in \mathbb{Z}^{*}$, the techniques from the proof of Lemma 3.18 lead to $H(\boldsymbol{\Theta})^{*}=D\left(F(\boldsymbol{\Theta})^{*}\right) \supseteq h^{\tilde{d}}\left(\mathbb{Z}^{*}, \mathbb{C}\right)$.

\subsection{Proof of Proposition 3.1}

Proof of Proposition 3.1. Let us introduce the following sequences:

$$
\begin{aligned}
\Theta:=\left(\theta_{k}\right)_{k \in \mathbb{Z}^{*}} \quad: \quad \theta_{k}=-\lambda_{k}, \quad \forall k>0 ; \quad \theta_{k}=\lambda_{-k}, \quad \forall k<0 ; \\
v:=\left(\nu_{k}\right)_{k \in \mathbb{Z}^{*}} \quad: \quad \nu_{k}=-\sqrt{\lambda_{k}}, \quad \forall k>0 ; \quad \nu_{k}=\sqrt{\lambda_{-k}}, \quad \forall k<0 ; \\
\alpha:=\left(\alpha_{k}\right)_{k \in \mathbb{Z}^{*} \backslash\{-1\}} \quad: \quad \alpha_{k}=-\lambda_{k}+\lambda_{1}, \quad \forall k>0 ; \quad \alpha_{k}=\lambda_{-k}-\lambda_{1}, \quad \forall k<-1 .
\end{aligned}
$$

We consider $\mathcal{M}^{\prime} \in \mathbb{N}^{*}$ and $\delta^{\prime}>0$ so that $\boldsymbol{\Theta}$ and $\boldsymbol{v}$ satisfy (11) with respect to $\mathcal{M}^{\prime}$ and $\delta^{\prime}$ (as in Remark 3.17), while

$$
\inf _{\left\{k \in \mathbb{Z}^{*} \backslash\{-1\}: k+\mathcal{M}^{\prime} \in \mathbb{Z}^{*} \backslash\{-1\}\right\}}\left|\alpha_{k+\mathcal{M}^{\prime}}-\alpha_{k}\right| \geq \delta^{\prime} \mathcal{M}^{\prime}
$$


Let $\left\{E_{m}\right\}_{m \in \mathbb{Z}^{*}}$ be the equivalence classes in $\mathbb{Z}^{*}$ defined by $\Theta$ and $\boldsymbol{~}$. Let us assume that $-1 \in E_{-1}$. Now, $\left\{E_{m}\right\}_{m \in \mathbb{Z}^{*} \backslash\{-1\}} \cup\left\{E_{-1} \backslash\{-1\}\right\}$ are the equivalence classes in $\mathbb{Z}^{*} \backslash\{-1\}$ defined by (19). Thanks to (9), the sequence $\boldsymbol{v}$ satisfies (18) and Remark 3.20 is valid with respect to the sequences $\boldsymbol{\Theta}$ and $\boldsymbol{v}$. Thus, $H(\boldsymbol{\Theta})^{*} \supseteq h^{\tilde{d}}\left(\mathbb{Z}^{*}, \mathbb{C}\right)$. We define as in Section 3.3, the operator $F(\alpha)$ in $\ell^{2}\left(\mathbb{Z}^{*} \backslash\{-1\}, \mathbb{C}\right)$ from the sequence $\alpha$ and we notice that, for every $m \neq-1$, we have $F_{m}\left(\alpha^{m}\right)=F_{m}\left(\boldsymbol{\Theta}^{m}\right)$ and $F_{m}\left(\alpha^{m}\right)^{*}=F_{m}\left(\boldsymbol{\Theta}^{m}\right)^{*}$. Thus, as in Lemma 3.19 and Remark 3.20, there hold

$$
H(\alpha) \supseteq h^{\tilde{d}}\left(\mathbb{Z}^{*} \backslash\{-1\}, \mathbb{C}\right), \quad H(\alpha)^{*} \supseteq h^{\tilde{d}}\left(\mathbb{Z}^{*} \backslash\{-1\}, \mathbb{C}\right) .
$$

We define $\mathbf{e}:=\left(e^{i \alpha_{k}(\cdot)}\right)_{k \in \mathbb{Z}^{*} \backslash\{-1\}}$ and $\boldsymbol{\Xi}:=\left(\xi_{k}\right)_{k \in \mathbb{Z}^{*} \backslash\{-1\}}=F(\alpha)^{*} \mathbf{e}$. When $T>2 \pi / \delta^{\prime}$, Theorem 3.12 ensures that $\left(\xi_{k}\right)_{k \in \mathbb{Z}^{*} \backslash\{-1\}}$ is a Riesz Basis of the space $X:=\overline{\operatorname{span}\left\{\xi_{k}: k \in \mathbb{Z}^{*} \backslash\{-1\}\right\}}{ }^{2}$. Now, the map

$$
M: g \in X \longmapsto\left(\left\langle\xi_{k}, g\right\rangle_{L^{2}(0, T)}\right)_{k \in \mathbb{Z}^{*} \backslash\{-1\}} \in \ell^{2}\left(\mathbb{Z}^{*} \backslash\{-1\}, \mathbb{C}\right)
$$

is invertible thanks to Proposition 3.9. Denoted $\tilde{X}:=M^{-1} \circ F(\alpha)^{*}\left(h^{\widetilde{d}}\left(\mathbb{Z}^{*} \backslash\{-1\}, \mathbb{C}\right)\right)$, the map

$$
\left(F(\alpha)^{*}\right)^{-1} \circ M: g \in \tilde{X} \longmapsto\left(\langle\mathbf{e}, g\rangle_{L^{2}(0, T)}\right)_{k \in \mathbb{Z}^{*} \backslash\{-1\}} \in h^{\tilde{d}}\left(\mathbb{Z}^{*} \backslash\{-1\}, \mathbb{C}\right)
$$

is invertible. Thus, for every $\left(x_{k}\right)_{k \in \mathbb{Z}^{*} \backslash\{-1\}} \in h^{\tilde{d}}\left(\mathbb{Z}^{*} \backslash\{-1\}, \mathbb{C}\right)$, there exists $u \in X \subseteq L^{2}((0, T), \mathbb{C})$ such that

$$
x_{k}=\int_{0}^{T} u(\tau) e^{-i \alpha_{k} \tau} d \tau, \quad \forall k \in \mathbb{Z}^{*} \backslash\{-1\} .
$$

The last relation can be used to ensure the solvability of the moment problem $(10)$ for $u \in L^{2}((0, T), \mathbb{C})$. So, we need to proceed as follows in order to ensure the result for a function $u \in L^{2}((0, T), \mathbb{R})$.

Given $\mathbf{x}=\left(x_{k}\right)_{k \in \mathbb{N}^{*}} \in h^{\tilde{d}}\left(\mathbb{N}^{*}, \mathbb{C}\right)$ so that $x_{1} \in \mathbb{R}$, we introduce $\left(\tilde{x}_{k}\right)_{k \in \mathbb{Z}^{*} \backslash\{-1\}} \in h^{\tilde{d}}\left(\mathbb{Z}^{*} \backslash\{-1\}, \mathbb{C}\right)$ so that $\tilde{x}_{k}=x_{k}$ for $k>0$, while $\tilde{x}_{k}=\bar{x}_{-k}$ for $k<-1$. Thanks to (20) and to the definition of $\alpha$, there exists $u \in X \subseteq L^{2}((0, T), \mathbb{C})$ so that

$$
\begin{array}{cc}
\int_{0}^{T} u(s) e^{i\left(\lambda_{k}-\lambda_{1}\right) s} d s=x_{k}=\int_{0}^{T} \bar{u}(s) e^{i\left(\lambda_{k}-\lambda_{1}\right) s} d s, & k \in \mathbb{N}^{*} \backslash\{1\}, \\
\int_{0}^{T} \bar{u}(s) e^{-i\left(\lambda_{k}-\lambda_{1}\right) s} d s=\overline{x_{k}}=\int_{0}^{T} u(s) e^{-i\left(\lambda_{k}-\lambda_{1}\right) s} d s, & k \in \mathbb{N}^{*} \backslash\{1\},
\end{array}
$$

while $\int_{0}^{T} u(s) d s=x_{1}$. The relations $(21)$ and the fact that $x_{1} \in \mathbb{R}$ imply $\left\langle\operatorname{Im}(u), e^{i \alpha_{k}(\cdot)}\right\rangle_{L^{2}(0, T)}=0$ for every $k \in \mathbb{Z}^{*} \backslash\{-1\}$ and then

$$
\left\langle\operatorname{Im}(u), \xi_{k}\right\rangle_{L^{2}(0, T)}=\left(F(\alpha)^{*}\left(\left\langle\operatorname{Im}(u), e^{i \alpha_{l}(\cdot)}\right\rangle_{L^{2}(0, T)}\right)_{l \in \mathbb{Z}^{*} \backslash\{-1\}}\right)_{k}=0, \quad \forall k \in \mathbb{Z}^{*} \backslash\{-1\} .
$$

From Remark 3.13, the family $\left\{e^{i \alpha_{k} t}\right\}_{k \in \mathbb{Z}^{*} \backslash\{-1\}}$ is minimal in $X$ and $X=\overline{\operatorname{span}\left\{e^{i \alpha_{k} t}: k \in \mathbb{Z}^{*} \backslash\{-1\}\right\}} L^{L^{2}}$. Now, we recall that $-\alpha_{k}=\alpha_{-k}$ for every $k \in \mathbb{Z}^{*} \backslash\{ \pm 1\}$ and $\alpha_{1}=0$. For every $u \in X$, we have

$$
\bar{u} \in{\overline{\operatorname{span}\left\{e^{-i \alpha_{k} t}: k \in \mathbb{Z}^{*} \backslash\{-1\}\right\}}}^{L^{2}}={\overline{\operatorname{span}\left\{e^{i \alpha_{k} t}: k \in \mathbb{Z}^{*} \backslash\{-1\}\right\}}}^{L^{2}}=X,
$$

which implies that $\operatorname{Im}(u)=\frac{u-\bar{u}}{2 i} \in X$. We call $\mathbf{v}:=\left(v_{k}\right)_{k \in \mathbb{Z}^{*} \backslash\{-1\}}$ the biorthogonal sequence to $\boldsymbol{\Xi}$ in $X$ which is also a Riesz basis of $X$ (see Remark 3.8). Now, for every $u \in X$, we have $\operatorname{Im}(u) \in X$ and

$$
\operatorname{Im}(u)=\sum_{k \in \mathbb{Z}^{*} \backslash\{-1\}} v_{k} c_{k}, \quad \text { for } \quad c_{k}=\left\langle\operatorname{Im}(u), \xi_{k}\right\rangle_{L^{2}(0, T)}, \quad \forall k \in \mathbb{Z}^{*} \backslash\{-1\} .
$$

In conclusion, when $u$ satisfies (21), the identities (22) and (23) yield that $\operatorname{Im}(u)=0$ and then $u$ is real.

Remark 3.21. The hypotheses on the function $G$ in Proposition 3.1 can be rewritten in terms of the sequence $\left\{ \pm \lambda_{k}\right\}_{k \in \mathbb{N}^{*}}$ rather than $\left\{ \pm \sqrt{\lambda_{k}}\right\}_{k \in \mathbb{N}^{*}}$. In this case, one can ensure the solvability of the moment problem (10) for sequences in $h^{1+\tilde{d}}\left(\mathbb{N}^{*}, \mathbb{C}\right)$ for every $T>0$. Indeed, if Lemma 3.16 is valid for $v=$ $\left\{ \pm \lambda_{k}\right\}_{k \in \mathbb{N}^{*}}$, then $H(v)^{*} \supseteq h^{1+\tilde{d}}\left(\mathbb{N}^{*}, \mathbb{C}\right)$ as for Lemma 3.19 and the solvability of $(10)$ in $h^{1+\tilde{d}}\left(\mathbb{N}^{*}, \mathbb{C}\right)$ is guaranteed by the techniques adopted in the proof of Proposition 3.1. Nevertheless, such result presents some disadvantages which make it unsuitable for our purposes as we explain in Remark 4.4. 


\section{Proof of Theorem 2.3}

\subsection{Local exact controllability}

For $\varepsilon, T>0$, we introduce the following subspaces of $H_{\mathscr{G}}^{s}$ with $s>0$ :

$$
O_{\varepsilon, T}^{s}:=\left\{\psi \in H_{\mathscr{G}}^{s} \mid\|\psi\|_{L^{2}}=1,\left\|\psi-\phi_{1}(T)\right\|_{(s)}<\varepsilon\right\}, \quad \phi_{1}(T):=e^{-i \lambda_{1} T} \phi_{1} .
$$

Definition 4.1. The (BSE) is said to be locally exactly controllable in $O_{\varepsilon, T}^{s}$ for $\varepsilon, T, s>0$ when, for every $\psi \in O_{\varepsilon, T}^{s}$, there exists $u \in L^{2}((0, T), \mathbb{R})$ such that $\psi=\Gamma_{T}^{u} \phi_{1}$.

Proposition 4.2. Let the hypotheses of Theorem 2.3 be satisfied. The (BSE) is locally exactly controllable in $O_{\varepsilon, T}^{s}$ for $\varepsilon>0$ sufficiently small, $T>0$ sufficiently large and $s=2+d$ with d from Assumptions $I I(\eta, \tilde{d})$.

Proof. The result corresponds to the surjectivity of the map $\Gamma_{T}^{(\cdot)} \phi_{1}: L^{2}((0, T), \mathbb{R}) \longrightarrow O_{\varepsilon, T}^{s}$. Let us define $\phi_{k}(T):=e^{-i \lambda_{k} T} \phi_{k}$ with $k \in \mathbb{N}^{*}$. We decompose $\Gamma_{T}^{(\cdot)} \phi_{1}=\sum_{k \in \mathbb{N}^{*}} \phi_{k}(T)\left\langle\phi_{k}(T), \Gamma_{T}^{(\cdot)} \phi_{1}\right\rangle_{L^{2}}$ and we consider

$$
\alpha: u \in L^{2}((0, T), \mathbb{R}) \longmapsto\left(\left\langle\phi_{k}(T), \Gamma_{T}^{u} \phi_{1}\right\rangle_{L^{2}}\right)_{k \in \mathbb{N}^{*}} .
$$

Now, $\Gamma_{T}^{u} \phi_{1} \in H_{\mathscr{G}}^{s}$ for every $u \in L^{2}((0, T), \mathbb{R})$ and $T>0$ thanks to Proposition 2.1. Thus, $\alpha$ takes value in $Q^{s}:=\left\{\mathbf{x}:=\left(x_{k}\right)_{k \in \mathbb{N}^{*}} \in h^{s}(\mathbb{C}) \mid\|\mathbf{x}\|_{\ell^{2}}=1\right\}$. Defined $\delta:=\left(\delta_{k, 1}\right)_{k \in \mathbb{N}^{*}}$, we notice that

$$
\alpha(0)=\left(\left\langle\phi_{k}(T), \Gamma_{T}^{0} \phi_{1}\right\rangle_{L^{2}}\right)_{k \in \mathbb{N}^{*}}=\left(\left\langle\phi_{k}(T), \phi_{1}(T)\right\rangle_{L^{2}}\right)_{k \in \mathbb{N}^{*}}=\delta .
$$

The local exact controllability of the (BSE) in $O_{\varepsilon, T}^{s}$ is equivalent to the surjectivity of the function

$$
\alpha: L^{2}((0, T), \mathbb{R}) \longrightarrow Q_{\varepsilon}^{s}:=\left\{\mathbf{x}:=\left(x_{k}\right)_{k \in \mathbb{N}^{*}} \in Q^{s} \mid\|\mathbf{x}-\delta\|_{(s)}<\varepsilon\right\} .
$$

Let $T_{\delta} Q^{s}$ be the tangent space of $Q^{s}$ in the point $\alpha(0)=\delta$. For every $f:[0,1] \rightarrow Q^{s}$ such that $f(0)=\delta$ and $f^{\prime}(0)=\mathbf{x} \in h^{s}(\mathbb{C})$, we have $0=\left(\partial_{t}\langle f(t), f(t)\rangle\right)(t=0)=2 \Re\left(\langle\mathbf{x}, \delta\rangle_{\ell^{2}}\right)$, which implies

$$
T_{\delta} Q^{s}=\left\{\mathbf{x}:=\left(x_{k}\right)_{k \in \mathbb{N}^{*}} \in h^{s}(\mathbb{C}) \mid i x_{1} \in \mathbb{R}\right\} .
$$

Let $P$ be the orthogonal projector onto $T_{\delta} Q^{s}$. We define $\widetilde{Q}^{s}:=\left\{\mathbf{x}:=\left(x_{k}\right)_{k \in \mathbb{N}^{*}} \in h^{s}(\mathbb{C}) \mid\|\mathbf{x}\|_{\ell^{2}} \leq 1\right\}$ and $\widetilde{Q}_{\varepsilon}^{s}:=\left\{\mathbf{x}:=\left(x_{k}\right)_{k \in \mathbb{N}^{*}} \in \widetilde{Q}^{s} \mid\|\mathbf{x}\|_{(s)}<\varepsilon\right\}$. We consider the Fréchet derivative of $\alpha$ in $u=0$ :

$$
\gamma: v \in L^{2}((0, T), \mathbb{R}) \longmapsto\left(d_{u} \alpha(u=0)\right) \cdot v \in T_{\delta} Q^{s} .
$$

We notice that $P \alpha: L^{2}((0, T), \mathbb{R}) \rightarrow \widetilde{Q}^{s} \cap T_{\delta} Q^{s}$ and its Fréchet derivative in $u=0$ is $\gamma$. If $\gamma$ is surjective in $T_{\delta} Q^{s}$, then the Generalized Inverse Function Theorem ([Lue69, Theorem 1; p. 240]) guarantees the existence of $\varepsilon>0$ sufficiently small so that $P \alpha$ is surjective in $\widetilde{Q}_{\varepsilon}^{s} \cap T_{\delta} Q^{s}$. Now, for every $\mathbf{x}=\left\{x_{k}\right\}_{k \in \mathbb{N}^{*}} \in$ $Q_{\epsilon}^{s}$, we have $P \mathbf{x} \in \widetilde{Q}_{\epsilon}^{s}$ and if there exists $u \in L^{2}((0, T), \mathbb{R})$ such that $P \mathbf{x}=P \alpha(u)$, then

$$
\mathbf{x}=P \mathbf{x}+\sqrt{1-\|P \mathbf{x}\|_{\ell^{2}}^{2}} \delta=P \alpha(u)+\sqrt{1-\|P \alpha(u)\|_{\ell^{2}}^{2}} \delta=\alpha(u) .
$$

The last relation and the Generalized Inverse Function Theorem imply that if $\gamma$ is surjective in $T_{\delta} Q^{s}$, then $\alpha$ is surjective in $Q_{\epsilon}^{s}$ with $\epsilon>0$ sufficiently small. Thus, we study the function $\gamma$ and, thanks to the Duhamel's formula provided in (6), we notice that it is composed by the elements

$$
\gamma_{k}(v)=-i\left\langle e^{-i \lambda_{k} T} \phi_{k}, \int_{0}^{T} e^{-i A(T-\tau)} v(\tau) B e^{-i \lambda_{1} \tau} \phi_{1} d \tau\right\rangle_{L^{2}}=-i \int_{0}^{T} v(\tau) e^{i\left(\lambda_{k}-\lambda_{1}\right) \tau} d \tau\left\langle\phi_{k}, B \phi_{1}\right\rangle_{L^{2}} .
$$

Proving the surjectivity of $\gamma$ corresponds to ensure the solvability of the following moment problem

$$
x_{k}\left\langle\phi_{j}, B \phi_{k}\right\rangle_{L^{2}}^{-1}=-i \int_{0}^{T} u(\tau) e^{i\left(\lambda_{k}-\lambda_{1}\right) \tau} d \tau, \quad \forall k \in \mathbb{N}^{*}
$$

for every $\left(x_{k}\right)_{k \in \mathbb{N}^{*}} \in T_{\delta} Q^{s}$. We notice that $\left(x_{k}\left\langle\phi_{k}, B \phi_{1}\right\rangle_{L^{2}}^{-1}\right)_{k \in \mathbb{N}^{*}} \in h^{s-2-\eta}=h^{d-\eta} \subseteq h^{\tilde{d}}$ thanks to the point 1. of Assumptions $\mathrm{I}(\eta)$. As $B$ is symmetric, we have $\left\langle\phi_{1}, B \phi_{1}\right\rangle_{L^{2}} \in \mathbb{R}$ and $i x_{1}\left\langle\phi_{1}, B \phi_{1}\right\rangle_{L^{2}}^{-1} \in \mathbb{R}$. Thanks to [DZ06, Proposition 6.2], there exist $\delta>0$ and $\mathcal{M} \in \mathbb{N}^{*}$ such that

$$
\inf _{k \in \mathbb{N}^{*}}\left|\sqrt{\lambda_{k+\mathcal{M}}}-\sqrt{\lambda_{k}}\right| \geq \delta \mathcal{M}
$$

The last relation and the identity (5) ensure that Proposition 3.1 is satisfied and the solvability of (24) is guaranteed in $\left\{\left(c_{k}\right)_{k \in \mathbb{N}^{*}} \in h^{\tilde{d}}(\mathbb{C}) \mid i c_{1} \in \mathbb{R}\right\}$ for $T>0$ large enough. As $\left(x_{k}\left\langle\phi_{k}, B \phi_{1}\right\rangle_{L^{2}}^{-1}\right)_{k \in \mathbb{N}^{*}}$ belongs to such space for every $\left(x_{k}\right)_{k \in \mathbb{N}^{*}} \in T_{\delta} Q^{s}$, the claim is proved. 
Remark 4.3. We consider the unitary propagator $\widetilde{\Gamma}_{t}^{u}$ generated by the time-dependent Hamiltonian $-A-$ $u(T-t) B$ with $u \in L^{2}((0, T), \mathbb{R})$ with $T>0$. As explained in [Duc20, Section 2.3], the propagator $\widetilde{\Gamma}_{t}^{u}$ represents the reversed dynamics of the (BSE) with the same $u$ and

$$
\left(\widetilde{\Gamma}_{T}^{u}\right)\left(\Gamma_{T}^{u}\right)=\left(\Gamma_{T}^{u}\right)\left(\widetilde{\Gamma}_{T}^{u}\right)=I
$$

When the hypotheses of Theorem 2.3 are satisfied, the local exact controllability results of Proposition 4.2 is also valid for the reversed dynamics. Indeed, the functions $\left(\phi_{k}\right)_{k \in \mathbb{N}^{*}}$ are eigenfunctions of $-A$ corresponding to the eigenvalues $\left(-\lambda_{k}\right)_{k \in \mathbb{N}^{*}}$. Let $s=2+d$ and $d$ from Assumptions $\operatorname{II}(\eta, \tilde{d})$. As in the proof of Proposition 4.2, the local exact controllability of the reversed dynamics in the neighborhood of $H_{\mathscr{G}}^{s}$ :

$$
\widetilde{O}_{\varepsilon, T}^{s}:=\left\{\psi \in H_{\mathscr{G}}^{s} \mid\|\psi\|_{L^{2}}=1,\left\|\psi-e^{i \lambda_{1} T} \phi_{1}\right\|_{(s)}<\varepsilon\right\},
$$

with $\varepsilon, T>0$ can be ensured by proving the solvability of a moment problem of the form

$$
x_{k}\left\langle\phi_{j}, B \phi_{k}\right\rangle_{L^{2}}^{-1}=-i \int_{0}^{T} u(\tau) e^{-i\left(\lambda_{k}-\lambda_{1}\right) \tau} d \tau, \quad \forall k \in \mathbb{N}^{*}
$$

for every $\left(x_{k}\right)_{k \in \mathbb{N}^{*}} \in T_{\delta} Q^{s}$. The result is proved as Proposition 4.2 thanks to Remark 3.2. Hence, for $\varepsilon>0$ sufficiently small and $T>0$ sufficiently large, we have that for every $\psi \in \widetilde{O}_{\varepsilon, T}^{s}$, there exists $u \in L^{2}((0, T), \mathbb{R})$ such that $\psi=\widetilde{\Gamma}_{T}^{u} \phi_{1}$. Finally, the last relation yields that

$$
\phi_{1}=\Gamma_{T}^{\widetilde{u}} \psi \quad \text { for } \quad \widetilde{u}(t)=u(T-t) .
$$

\subsection{Global exact controllability}

Proof of Theorem 2.3. Let us consider $\psi_{1}, \psi_{2} \in H_{\mathscr{G}}^{s}$ be so that $\left\|\psi_{1}\right\|_{L^{2}}=\left\|\psi_{2}\right\|_{L^{2}}$. We assume that $\left\|\psi_{1}\right\|_{L^{2}}=\left\|\psi_{2}\right\|_{L^{2}}=1$, but the result is equivalently proved in the general case. Let $T, \varepsilon>0$ be so that Proposition 4.2 and Remark 4.3 are valid. Thanks to Proposition A.2 and Corollary A.3, there exist $T_{1}, T_{2}>0, u_{1} \in L^{2}\left(\left(0, T_{1}\right), \mathbb{R}\right)$ and $u_{2} \in L^{2}\left(\left(0, T_{2}\right), \mathbb{R}\right)$ such that

$$
\left\|\Gamma_{T_{1}}^{u_{1}} \psi_{1}-e^{i \lambda_{1} T} \phi_{1}\right\|_{(s)}<\varepsilon, \quad\left\|\widetilde{\Gamma}_{T_{2}}^{u_{2}} \psi_{2}-e^{-i \lambda_{1} T} \phi_{1}\right\|_{(s)}<\varepsilon, \quad \Longrightarrow \quad \Gamma_{T_{1}}^{u_{1}} \psi_{1} \in \widetilde{O}_{\varepsilon, T}^{s}, \widetilde{\Gamma}_{T_{2}}^{u_{2}} \psi_{2} \in O_{\varepsilon, T}^{s} .
$$

From Remark 4.3 (relation $(26)$ ), there exists $u_{3} \in L^{2}((0, T), \mathbb{R})$ such that $\Gamma_{T}^{u_{3}} \Gamma_{T_{1}}^{u_{1}} \psi_{1}=\phi_{1}$. From Proposition 4.2 , there exist $u_{4} \in L^{2}((0, T), \mathbb{R})$ such that $\Gamma_{T}^{u_{4}} \phi_{1}=\widetilde{\Gamma}_{T_{2}}^{u_{2}} \psi_{2}$ and then

$$
\Gamma_{T}^{u_{4}} \Gamma_{T}^{u_{3}} \Gamma_{T_{1}}^{u_{1}} \psi_{1}=\widetilde{\Gamma}_{T_{2}}^{u_{2}} \psi_{2} \quad \Longrightarrow \quad \Gamma_{T_{2}}^{\widetilde{u}_{2}} \Gamma_{T}^{u_{4}} \Gamma_{T}^{u_{3}} \Gamma_{T_{1}}^{u_{1}} \psi_{1}=\psi_{2}, \quad \text { for } \quad \widetilde{u}_{2}(t)=u_{2}\left(T_{2}-t\right) .
$$

In conclusion, Theorem 2.3 is proved since there exists $\widehat{T}>0$ and $\widehat{u} \in L^{2}((0, \widehat{T}), \mathbb{R})$ such that $\Gamma_{\widehat{T}}^{\widehat{u}} \psi_{1}=$ $\psi_{2}$.

Remark 4.4. As discussed in Remark 3.21, one can ensure the solvability of (24) for sequences in $h^{1+\tilde{d}}\left(\mathbb{N}^{*}, \mathbb{C}\right)$ for every $T>0$ by changing the hypotheses on the function $G$. This outcome leads to the local exact controllability of Proposition 4.2 for any $T>0$. Nevertheless, the new conditions on $G$ and the solvability of the moment problem in $h^{1+\tilde{d}}\left(\mathbb{N}^{*}, \mathbb{C}\right)$, rather than $h^{\tilde{d}}\left(\mathbb{N}^{*}, \mathbb{C}\right)$, compel to impose stronger hypotheses on the problem. Our choice allows us to treat a wide range of problems as Theorem 1.3 and Theorem 1.4 which would be otherwise out of reach. We also point out that, when we extend the local exact controllability in order to prove Theorem 2.3, the property of being controllable in any positive time is lost.

\section{Bilinear quantum systems on star graphs}

In the current section, we study the global exact controllability when $\mathscr{G}$ is a star graph by applying Theorem 2.3. The result is obtained by providing a suitable entire function $G$ satisfying the hypotheses of the theorem. From now on, when we call $\mathscr{G}$ a star graph, we also consider it as a quantum graph.

Theorem 5.1. Let $\mathscr{G}$ be a star graph equipped with $(\mathcal{D} / \mathcal{N})$ made by edges of lengths $\left\{L_{j}\right\}_{j \leq N} \in \mathcal{A L}(N)$. If the couple $(A, B)$ satisfies Assumptions $I(\eta)$ and Assumptions $I I(\eta, \epsilon)$ for $\eta, \epsilon>0$, then the $(B S E)$ is globally exactly controllable in $H_{\mathscr{G}}^{s}$ for $s=2+d$ and $d$ from Assumptions $\operatorname{II}(\eta, \epsilon)$. 
Proof. 1) Star graph equipped with $(\mathcal{D})$. The boundary conditions $(\mathcal{D})$ on $V_{e}$ imply that $\phi_{k}=$ $\left(a_{k}^{1} \sin \left(\sqrt{\lambda_{k}} x\right), \ldots, a_{k}^{n} \sin \left(\sqrt{\lambda_{k}} x\right)\right)$ for each $k \in \mathbb{N}^{*}$ and suitable $\left\{a_{k}^{l}\right\}_{l \leq N} \subset \mathbb{C}$ such that $\left(\phi_{k}\right)_{k \in \mathbb{N}^{*}}$ is orthormal in $\mathscr{H}$. The conditions $(\mathcal{N} \mathcal{K})$ in the internal vertex $v \in V_{i}$ ensure that, for every $k \in \mathbb{N}^{*}$,

$$
\left\{\begin{array}{l}
a_{k}^{1} \sin \left(\sqrt{\lambda_{k}} L_{1}\right)=\ldots=a_{k}^{N} \sin \left(\sqrt{\lambda_{k}} L_{N}\right), \quad \Longrightarrow \quad \sum_{l=1}^{N} \cot \left(\sqrt{\lambda_{k}} L_{l}\right)=0 . \\
\sum_{l \leq N} a_{k}^{l} \cos \left(\sqrt{\lambda_{k}} L_{l}\right)=0,
\end{array}\right.
$$

We use the provided identities in order to construct an entire function satisfying the hypotheses of Theorem 2.3. To this purpose, we define an entire function $G$ and two maps $\tilde{G}$ and $H$ such that

$$
\begin{array}{rlrl}
G(x) & :=\prod_{l \leq N} \sin \left(x L_{l}\right) \sum_{l \leq N} \cot \left(x L_{l}\right) & G^{\prime}(x) & =-\tilde{G}(x)+H(x), \\
\tilde{G}(x) & :=\prod_{l \leq N} \sin \left(x L_{l}\right) \sum_{l \leq N} \frac{L_{l}}{\sin ^{2}\left(x L_{l}\right)}, & H(x):=\frac{d}{d x}\left(\prod_{l \leq N} \cos \left(x L_{l}\right)\right) \sum_{l \leq N} \cot \left(x L_{l}\right) .
\end{array}
$$

The identities (27) and (28) imply that $H\left(\sqrt{\lambda_{k}}\right)=0$ and $G^{\prime}\left(\sqrt{\lambda_{k}}\right)=-\tilde{G}\left(\sqrt{\lambda_{k}}\right)$ for every $k \in \mathbb{N}^{*}$. Now,

$$
|\tilde{G}(x)|=\frac{\prod_{l \leq N}\left|\sin \left(x L_{l}\right)\right| \sum_{l \leq N} L_{l} \prod_{k \neq l} \sin ^{2}\left(x L_{k}\right)}{\prod_{l \leq N} \sin ^{2}\left(x L_{l}\right)} \geq L^{*} \sum_{l \leq N} \prod_{k \neq l}\left|\sin \left(x L_{k}\right)\right|,
$$

with $L^{*}:=\min _{l \leq N} L_{l}$. Thanks to (29), we refer to [DZ06, Corollary A.10; (2)] (which contains a misprint as it is valid for $\left.\lambda>\frac{\pi}{2} \max \left\{1 / L_{j}: j \leq N\right\}\right)$ and for every $\epsilon>0$, there exists $C_{1}>0$ such that

$$
\left|G^{\prime}\left( \pm \sqrt{\lambda_{k}}\right)\right| \geq L^{*} \sum_{l=1}^{N} \prod_{j \neq l}\left|\sin \left(\sqrt{\lambda_{k}} L_{j}\right)\right| \geq \frac{C_{1}}{\left(\sqrt{\lambda_{k}}\right)^{1+\epsilon}}, \quad \forall k \in \mathbb{N}^{*} \quad: \quad \lambda_{k}>\frac{\pi}{2} \max \left\{L_{j}^{-1}\right\}_{j \leq N} .
$$

Remark 5.2. For every $k \in \mathbb{N}^{*}$ and $j \leq N$, we have $\left|\phi_{k}^{j}\left(L_{j}\right)\right| \neq 0$, otherwise the $(\mathcal{N} \mathcal{K})$ conditions would ensure that $\phi_{k}^{l}\left(L_{l}\right)=\phi_{k}^{m}\left(L_{m}\right)=0$ with $l, m \leq N$ so that $\phi_{k}^{l}, \phi_{k}^{m} \not \equiv 0$ and there would be satisfied $a_{k}^{l} \sin \left(L_{l} \sqrt{\lambda_{k}}\right)=a_{k}^{m} \sin \left(L_{m} \sqrt{\lambda_{k}}\right)=0$ with $a_{k}^{l}, a_{k}^{m} \neq 0$, which is absurd as $\left\{L_{j}\right\}_{j \leq N} \in \mathcal{A L}(N)$.

Remark 5.2 implies $\left|G^{\prime}\left( \pm \sqrt{\lambda_{k}}\right)\right| \neq 0$ for $k \in \mathbb{N}^{*}$. Thanks to (5) and (30), there exists $C_{2}>0$ so that

$$
\left|G^{\prime}\left( \pm \sqrt{\lambda_{k}}\right)\right| \geq C_{2} k^{-(1+\epsilon)}, \quad \forall k \in \mathbb{N}^{*} .
$$

We notice that the spectrum of $A$ is simple. Indeed, if there would exist two orthonormal eigenfuctions $f$ and $g$ of $A$ corresponding to the same eigenvalue $\lambda$, then $h(x)=f(v) g(x)-g(v) f(x)$ would be another eigenfunction of $A$. Now, $h$ is an eigenfunction and $h(v)=0$, which is impossible thanks to Remark 5.2.

As $\left|\cos \left(z L_{l}\right)\right| \leq e^{L_{l}|z|}$ and $\left|\sin \left(z L_{l}\right)\right| \leq e^{L_{l}|z|}$ for every $l \leq N$ and $z \in \mathbb{C}$, we notice that $|G(z)| \leq$ $N e^{|z| \sum_{l=1}^{N} L_{l}}$ for every $z \in \mathbb{C}$. Now, $G\left(\sqrt{\lambda_{k}}\right)=0$ for every $k \in \mathbb{N}^{*}$ thanks to $(27)$ and $G \in L^{\infty}(\mathbb{R}, \mathbb{R})$.

In conclusion, the claim is achieved as Theorem 2.3 is valid with respect to the function $G$ when $\tilde{d}=\epsilon$.

2) Generic star graph. Let $I_{1} \subseteq\{1, \ldots, N\}$ be the set of indices of those edges containing an external vertex equipped with $(\mathcal{N})$ and $I_{2}:=\{1, . ., N\} \backslash I_{1}$. The proof follows from the techniques adopted in $\left.\mathbf{1}\right)$ by considering Proposition B.2 (rather than [DZ06, Corollary A.10; (2)]) and the entire map

$$
G(x):=\prod_{l \in I_{2}} \sin \left(x L_{l}\right) \prod_{l \in I_{1}} \cos \left(x L_{l}\right)\left(\sum_{l \in I_{2}} \cot \left(x L_{l}\right)+\sum_{l \in I_{1}} \tan \left(x L_{l}\right)\right) .
$$

Remark 5.3. When $\mathscr{G}$ is a star graph equipped with $(\mathcal{D})$ such that $L_{2} / L_{1}$ and $L_{3} / L_{1}$ are rationals,

$$
\exists n_{2}, n_{3}, m_{2}, m_{3} \in \mathbb{N}^{*} \quad: \quad L_{2} / L_{1}=n_{2} / m_{2}, \quad L_{3} / L_{1}=n_{3} / m_{3} .
$$

The numbers $\left\{\mu_{k}\right\}_{k \in \mathbb{N}^{*}}$ with $\mu_{k}=\frac{k^{2} m_{2}^{2} m_{3}^{2} \pi^{2}}{L_{1}^{2}}$ are eigenvalues of $A$ and they are multiple. Fixed $k \in \mathbb{N}^{*}$,

$$
f_{k}=\left(-2 \sin \left(\sqrt{\mu}_{k} x\right), \sin \left(\sqrt{\mu}_{k} x\right), \sin \left(\sqrt{\mu}_{k} x\right), 0, \ldots, 0\right), \quad g_{k}=\left(0, \sin \left(\sqrt{\mu}_{k} x\right),-\sin \left(\sqrt{\mu}_{k} x\right), 0, \ldots, 0\right)
$$

are reciprocally orthogonal eigenfunctions of $A$ corresponding to $\mu_{k}$. In addition, we notice that the sequence $\left\{g_{k}\right\}_{k \in \mathbb{N}^{*}}$ is composed by eigenfuctions vanishing in the edge $e_{1}$. The same kind of construction can be repeated when the star graph $\mathscr{G}$ is equipped with the general boundary conditions $(\mathcal{D} / \mathcal{N})$. 
Corollary 5.4. Let $\mathscr{G}$ be a star graph equipped with $(\mathcal{D} / \mathcal{N})$. Let $\mathscr{G}$ satisfy the following conditions with $\widetilde{N} \in 2 \mathbb{N}^{*}$ such that $\widetilde{N} \leq N$.

- For $j \leq \widetilde{N} / 2$, the two external vertices belonging to $e_{2 j-1}$ and $e_{2 j}$ are equipped with $(\mathcal{D})$ or $(\mathcal{N})$.

- The couples of edges $\left\{e_{2 j-1}, e_{2 j}\right\}_{j \leq \widetilde{N} / 2}$ are long $\left\{L_{j}\right\}_{j \leq \widetilde{N} / 2}$, while the edges $\left\{e_{j}\right\}_{\widetilde{N}<j \leq N}$ measure $\left\{L_{j}\right\}_{\widetilde{N}<j \leq N}$. In addition, $\left\{L_{j}\right\}_{j \leq \frac{\widetilde{N}}{2}} \cup\left\{L_{j}\right\}_{\widetilde{N}<j \leq N} \in \mathcal{A L}\left(\frac{\widetilde{N}}{2}+N-\widetilde{N}\right)$.

If $(A, B)$ satisfies Assumptions $I(\eta)$ and Assumptions $I I(\eta, \epsilon)$ for $\eta, \epsilon>0$, then the (BSE) is globally exactly controllable in $H_{\mathscr{G}}^{s}$ for $s=2+d$ and $d$ from Assumptions $I I(\eta, \epsilon)$.



Figure 4: Example of graph described in Corollary 5.4 with $\tilde{N}=4$ and $N=6$.

Proof. Let $I_{1} \subseteq\{1, \ldots, \widetilde{N} / 2\}$ be the set of $j$ such that $e_{2 j-1}$ and $e_{2 j}$ contain two external vertices of $\mathscr{G}$ equipped with $(\mathcal{N})$ and $I_{2}:=\{1, . ., \widetilde{N} / 2\} \backslash I_{1}$. Let $I_{3} \subseteq\{\widetilde{N}+1, \ldots, N\}$ be the set of $j$ such that $e_{j}$ contains an external vertex of $\mathscr{G}$ equipped with $(\mathcal{N})$ and $I_{4}:=\{\widetilde{N}+1, \ldots, N\} \backslash I_{3}$. Let

$$
\left(\lambda_{k}^{1}\right)_{k \in \mathbb{N}^{*}}:=\left(\frac{(2 k-1)^{2} \pi^{2}}{4 L_{j}^{2}}\right)_{\substack{j, k \in \mathbb{N}^{*} \\ j \in I_{1}}}, \quad\left(\lambda_{k}^{2}\right)_{k \in \mathbb{N}^{*}}:=\left(\frac{k^{2} \pi^{2}}{L_{j}^{2}}\right)_{\substack{j, k \in \mathbb{N}^{*} \\ j \in I_{2}}} .
$$

We notice that $\left(\lambda_{k}^{1}\right)_{k \in \mathbb{N}^{*}} \cup\left(\lambda_{k}^{2}\right)_{k \in \mathbb{N}^{*}} \subset\left(\lambda_{k}\right)_{k \in \mathbb{N}^{*}}$ are the only eigenvalues of $A$ corresponding to eigenfunctions vanishing in the internal vertex $v$. For every $f \in\left(\phi_{k}\right)_{k \in \mathbb{N}^{*}}$ of $A$ corresponding to an eigenvalue $\lambda \in\left(\lambda_{k}^{1}\right)_{k \in \mathbb{N}^{*}}$, $f$ is uniquely defined (up to multiplication for $\alpha \in \mathbb{C}$ such that $|\alpha|=1$ ) by the identities

$$
f^{2 j-1}(x)=-f^{2 j}(x)=\sqrt{L_{j}^{-1}} \cos (\sqrt{\lambda} x), \quad f^{l} \equiv 0, \quad \forall l \in\{1, \ldots, N\} \backslash\{2 j-1,2 j\} .
$$

The same property is valid for $\left(\lambda_{k}^{2}\right)_{k \in \mathbb{N}^{*}}$ and then, the eigenvalues $\left(\lambda_{k}^{1}\right)_{k \in \mathbb{N}^{*}} \cup\left(\lambda_{k}^{2}\right)_{k \in \mathbb{N}^{*}}$ are simple. In conclusion, the discrete spectrum of $A$ is simple since, if there would exist a multiple eigenvalue

$$
\lambda \in\left(\lambda_{k}\right)_{k \in \mathbb{N}^{*}} \backslash\left(\left(\lambda_{k}^{1}\right)_{k \in \mathbb{N}^{*}} \cup\left(\lambda_{k}^{2}\right)_{k \in \mathbb{N}^{*}}\right),
$$

then there would exist two orthonormal eigenfuctions $f$ and $g$ corresponding to the same eigenvalue $\lambda$. Now, $h(x)=f(v) g(x)-g(v) f(x)$ would be another eigenfunction corresponding to $\lambda$ such that $h(v)=0$, which is impossible as it would imply that $\lambda \in\left(\lambda_{k}^{1}\right)_{k \in \mathbb{N}^{*}} \cup\left(\lambda_{k}^{2}\right)_{k \in \mathbb{N}^{*}}$. Thus, $\left(\lambda_{k}\right)_{k \in \mathbb{N}^{*}}$ are simple eigenvalues. The remaining part of proof follows the one of Theorem 5.1 thanks to Proposition B.2 by considering

$$
G(x):=\prod_{l \in I_{2} \cup I_{4}} \sin \left(x L_{l}\right) \prod_{l \in I_{1} \cup I_{3}} \cos \left(x L_{l}\right)\left(2 \sum_{l \in I_{2}} \cot \left(x L_{l}\right)+2 \sum_{l \in I_{1}} \tan \left(x L_{l}\right)+\sum_{l \in I_{4}} \cot \left(x L_{l}\right)+\sum_{l \in I_{3}} \tan \left(x L_{l}\right)\right) .
$$

\subsection{Proofs of Theorem 1.3 and of Theorem 1.4}

Proof of Theorem 1.3. Theorem 1.3 is proved such as [Duc18, Theorem 1.2] that is stated for $N=4$. The only difference between the two results is the fact that Theorem 1.3 follows from Theorem 5.1 instead of [Duc18, Proposition 3.3], which is only valid for $N \leq 4$.

Proof of Theorem 1.4. The conditions $(\mathcal{N})$ in $V_{i}$ imply the existence, for every $k \in \mathbb{N}^{*}$, of $\left\{a_{k}^{l}\right\}_{l \leq N} \subset \mathbb{C}$ such that $\phi_{k}=\left(a_{k}^{1} \cos \left(x \sqrt{\lambda_{k}}\right), \ldots, a_{k}^{N} \cos \left(x \sqrt{\lambda_{k}}\right)\right)$. The coefficients $\left\{a_{k}^{l}\right\}_{l \leq N} \subset \mathbb{C}$ are so that $\left(\phi_{k}\right)_{k \in \mathbb{N}^{*}}$ forms a Hilbert basis of $\mathscr{H}$ and then

$$
1=\sum_{l \leq N} \int_{0}^{L_{l}}\left|a_{k}^{l}\right|^{2} \cos ^{2}\left(x \sqrt{\lambda_{k}}\right) d x=\sum_{l \leq N}\left|a_{k}^{l}\right|^{2}\left(\frac{L_{l}}{2}+\frac{\sin \left(2 L_{l} \sqrt{\lambda_{k}}\right)}{4 \sqrt{\lambda_{k}}}\right) .
$$


For every $k \in \mathbb{N}^{*}$, the $(\mathcal{N K})$ boundary conditions in $V_{i}$ ensure

$$
\begin{gathered}
a_{k}^{1} \cos \left(\sqrt{\lambda_{k}} L_{1}\right)=\ldots=a_{k}^{N} \cos \left(\sqrt{\lambda_{k}} L_{N}\right), \quad \sum_{l \leq N} a_{k}^{l} \sin \left(\sqrt{\lambda_{k}} L_{l}\right)=0, \\
\sum_{l \leq N} \tan \left(\sqrt{\lambda_{k}} L_{l}\right)=0, \quad \sum_{l \leq N}\left|a_{k}^{l}\right|^{2} \sin \left(2 L_{l} \sqrt{\lambda_{k}}\right)=0 .
\end{gathered}
$$

The last identities and (31) imply $1=\sum_{l=1}^{N}\left|a_{k}^{l}\right|^{2} L_{l} / 2$. Thanks to (32), we have $a_{k}^{l}=a_{k}^{1} \frac{\cos \left(\sqrt{\lambda_{k}} L_{1}\right)}{\cos \left(\sqrt{\lambda_{k}} L_{l}\right)}$ for $l \neq 1$ and $k \in \mathbb{N}^{*}$. Thus, $\left|a_{k}^{1}\right|^{2}\left(L_{1}+\sum_{l=2}^{N} L_{l} \frac{\cos ^{2}\left(\sqrt{\lambda_{k}} L_{1}\right)}{\cos ^{2}\left(\sqrt{\lambda_{k}} L_{l}\right)}\right)=2$ for every $k \in \mathbb{N}^{*}$ and

$$
\left|a_{k}^{1}\right|^{2}=2 \prod_{m=2}^{N} \cos ^{2}\left(\sqrt{\lambda_{k}} L_{m}\right)\left(\sum_{j=1}^{N} L_{j} \prod_{m \neq j} \cos ^{2}\left(\sqrt{\lambda_{k}} L_{m}\right)\right)^{-1} .
$$

Validation of Assumptions $\mathbf{I}(\mathbf{3}+\epsilon)$ with $\epsilon>\mathbf{0}$. For every $k \in \mathbb{N}^{*}$, thanks to the relation (32)

$$
\prod_{l \leq N} \cos \left(\sqrt{\lambda_{k}} L_{l}\right) \sum_{l \leq N} \tan \left(\sqrt{\lambda_{k}} L_{l}\right)=0, \quad \Longrightarrow \quad \sum_{l=1}^{N} \sin \left(\sqrt{\lambda_{k}} L_{l}\right) \prod_{m \neq l} \cos \left(\sqrt{\lambda_{k}} L_{m}\right)=0 .
$$

Thanks to the relation (5) and Corollary B.3, for every $\epsilon>0$, there exist $C_{1}, C_{2}>0$ such that,

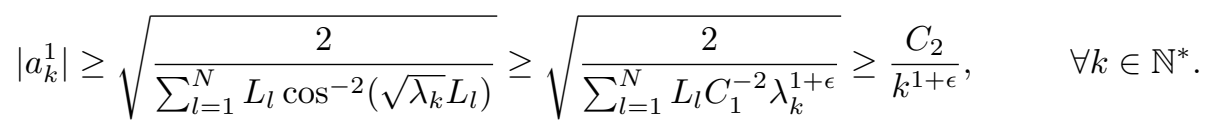

In addition, $\left\langle\phi_{1}^{l},\left(B \phi_{k}\right)^{l}\right\rangle_{L^{2}\left(e_{l}, \mathbb{C}\right)}=0$ for $2 \leq l \leq N$ and, for every $k \in \mathbb{N}^{*}$,

$$
\left\langle\phi_{1}, B \phi_{k}\right\rangle_{L^{2}}=\left\langle\phi_{1}^{1},\left(B \phi_{k}\right)^{1}\right\rangle_{L^{2}\left(e_{1}, \mathbb{C}\right)}=-\frac{120 a_{k}^{1} a_{1}^{1} L_{1}^{6}}{\left(\sqrt{\lambda_{k}}+\sqrt{\lambda_{1}}\right)^{4}}-\frac{120 a_{k}^{1} a_{1}^{1} L_{1}^{6}}{\left(\sqrt{\lambda_{k}}-\sqrt{\lambda_{1}}\right)^{4}}+o\left({\sqrt{\lambda_{k}}}^{-5}\right) .
$$

From the relations (33) and (34), thanks to the relation (5), for every $\epsilon>0$, there exists $C_{3}>0$, such that for $k \in \mathbb{N}^{*}$ sufficiently large,

$$
\left|\left\langle\phi_{1}, B \phi_{k}\right\rangle_{L^{2}}\right| \geq C_{3} k^{-(5+\epsilon)} .
$$

Now, it is possible to compute $a_{k}(\cdot)$ and $B_{k}(\cdot)$ with $k \in \mathbb{N}^{*}$, analytic functions in $\mathbb{R}^{+}$, so that

$$
a_{k}\left(L_{1}\right)^{2}=\left(a_{k}^{1}\right)^{2}, \quad a_{1}\left(L_{1}\right) a_{k}\left(L_{1}\right) B_{k}\left(L_{1}\right)=\left\langle\phi_{1}, B \phi_{k}\right\rangle_{L^{2}}
$$

and such that each $a_{1}(\cdot) a_{k}(\cdot) B_{k}(\cdot)$ is non-constant and analytic. Thus, each $a_{1}(\cdot) a_{k}(\cdot) B_{k}(\cdot)$ has discrete zeros $\tilde{V}_{k} \subset \mathbb{R}^{+}$and $\tilde{V}=\bigcup_{k \in \mathbb{N}^{*}} \tilde{V}_{k}$ is countable. For every $\left\{L_{l}\right\}_{l \leq N} \in \mathcal{A L}(N)$ so that $L_{1} \notin \tilde{V}$, we have $\left|\left\langle\phi_{1}, B \phi_{k}\right\rangle_{L^{2}}\right| \neq 0$ for every $k \in \mathbb{N}^{*}$. Thus, the point 1. of Assumptions $\mathrm{I}(3+\epsilon)$ is ensured thanks to the relations (35) since, for every $\epsilon>0$, there exists $C_{4}>0$ such that

$$
\left|\left\langle\phi_{1}, B \phi_{k}\right\rangle_{L^{2}}\right| \geq C_{4} k^{-(5+\epsilon)}, \quad \forall k \in \mathbb{N}^{*} .
$$

Let $(k, j),(m, n) \in I,(k, j) \neq(m, n)$ for $I:=\left\{(j, k) \in\left(\mathbb{N}^{*}\right)^{2}: j<k\right\}$. We prove the validity of the point 2. of Assumptions $\mathrm{I}(3+\epsilon)$. As above, we compute $F_{k}(\cdot)$ with $k \in \mathbb{N}^{*}$, analytic in $\mathbb{R}^{+}$, such that $\left\langle\phi_{k}, B \phi_{k}\right\rangle_{L^{2}}=F_{k}\left(L_{1}\right)$. Each $F_{j, k, l, m}(\cdot):=F_{j}(\cdot)-F_{k}(\cdot)-F_{l}(\cdot)+F_{m}(\cdot)$ is non-constant and analytic in $\mathbb{R}^{+}$, the set of its positive zeros $V_{j, k, l, m}$ is discrete. Now, we introduce the countable set:

$$
V:=\bigcup_{(j, k),(l, m) \in I:(j, k) \neq(l, m)} V_{j, k, l, m} .
$$

For $\left\{L_{l}\right\}_{l \leq N} \in \mathcal{A L}(N)$ so that $L_{1} \notin V \cup \tilde{V}$, the point 2. of Assumptions $\mathrm{I}(3+\epsilon)$ with $\epsilon>0$ is satisfied.

Validation of Assumptions II $\left(3+\epsilon_{1}, \epsilon_{2}\right)$ with $\epsilon_{\mathbf{1}}, \epsilon_{\mathbf{2}}>\mathbf{0}$ so that $\epsilon_{\mathbf{1}}+\epsilon_{\mathbf{2}} \in\left(\mathbf{0}, \frac{\mathbf{1}}{2}\right)$. Let

$$
P(x):=\left(5 x^{6}-24 x^{5} L_{1}+45 x^{4} L_{1}^{2}-40 x^{3} L_{1}^{3}+15 x^{2} L_{1}^{4}-L_{1}^{6}\right) .
$$

For $m>0$, we notice $B: H^{m} \longrightarrow H^{m}$ and $\partial_{x}(B \psi)(\widetilde{v})=0$ for every $\widetilde{v} \in V_{e}$ since $\partial_{x} P(0)=0$. Now, $\partial_{x}(B \psi)(v)=(B \psi)(v)=0$ with $v \in V_{i}$ since $\partial_{x} P\left(L_{1}\right)=P\left(L_{1}\right)=0$. Then, $B: H_{\mathscr{G}}^{2} \rightarrow H_{\mathscr{G}}^{2}$. Moreover, 
$\partial_{x}^{2} P\left(L_{1}\right)=\partial_{x}^{3} P\left(L_{1}\right)=0$, which implies $B: H_{\mathcal{N} \mathcal{K}}^{m} \longrightarrow H_{\mathcal{N} \mathcal{K}}^{m}$ for every $m \in\left(0, \frac{9}{2}\right)$. For $d \in\left[3+\epsilon_{1}+\epsilon_{2}, \frac{7}{2}\right)$ and $d_{1} \in\left(d, \frac{7}{2}\right)$, there follow

$$
\operatorname{Ran}\left(\left.B\right|_{H_{\mathcal{N K}}^{d_{1}}}\right) \subseteq H_{\mathcal{N} \mathcal{K}}^{d_{1}}, \quad \operatorname{Ran}\left(\left.B\right|_{H_{\mathscr{G}}^{2+d}}\right) \subseteq \operatorname{Ran}\left(\left.B\right|_{H^{2+d} \cap H_{\mathcal{N K}}^{1+d} \cap H_{\mathscr{G}}^{2}}\right) \subseteq H^{2+d} \cap H_{\mathcal{N K}}^{1+d} \cap H_{\mathscr{G}}^{2} .
$$

The point 2. of Assumptions $\operatorname{II}\left(3+\epsilon_{1}, \epsilon_{2}\right)$ with $\epsilon_{1}, \epsilon_{2}>0$ so that $\epsilon_{1}+\epsilon_{2} \in\left(0, \frac{1}{2}\right)$ is valid.

Conclusion. The couple $(A, B)$ satisfies Assumptions $\mathrm{I}(3+\epsilon)$ and Assumptions II $\left(3+\epsilon_{1}, \epsilon_{2}\right)$ with $\epsilon_{1}, \epsilon_{2}>0$ so that $\epsilon_{1}+\epsilon_{2} \in\left(0, \frac{1}{2}\right)$. Theorem 5.1 guarantees the global exact controllability of the $(B S E)$ in $H_{\mathscr{G}}^{s}$ with $s=2+d$ and $d \geq 3+\epsilon_{1}+\epsilon_{2}$.

\section{Energetic controllability}

We recall that $\left(\varphi_{k}\right)_{k \in \mathbb{N}^{*}} \subseteq\left(\phi_{k}\right)_{k \in \mathbb{N}^{*}}$ indicates an orthonormal system (not necessarily complete) of $\mathscr{H}$ made by some eigenfunctions of $A$ and $\left(\mu_{k}\right)_{k \in \mathbb{N}^{*}}$ the ordered sequence of corresponding eigenvalues. Let

$$
\widetilde{\mathscr{H}}:=\overline{\operatorname{span}\left\{\varphi_{k} \mid k \in \mathbb{N}^{*}\right\}} L^{2} .
$$

We refer to Definition 1.5 for the formal definition of energetic controllability.

Theorem 6.1. Let $\mathscr{G}$ be a compact quantum graph and one of the following points be verified.

1. There exists an entire function $G$ such that $G \in L^{\infty}(\mathbb{R}, \mathbb{R})$ and there exist $J, I>0$ so that $|G(z)| \leq$ $J e^{I|z|}$ for every $z \in \mathbb{C}$. The eigenvalues $\left\{\mu_{k}\right\}_{k \in \mathbb{N}^{*}}$ are simple, the numbers $\left\{ \pm \sqrt{\mu_{k}}\right\}_{k \in \mathbb{N}^{*}}$ are simple zeros of $G$ and there exist $\tilde{d} \geq 0$ and $C>0$ so that $\left|G^{\prime}\left( \pm \sqrt{\mu_{k}}\right)\right| \geq \frac{C}{k^{1+\tilde{d}}}$ for every $k \in \mathbb{N}^{*}$.

2. There exist $C>0$ and $\tilde{d} \geq 0$ so that $\left|\mu_{k+1}-\mu_{k}\right| \geq C k^{-\frac{\tilde{d}}{\mathcal{M}-1}}$ for each $k \in \mathbb{N}^{*}$ with $\mathcal{M}$ from (1).

If $(A, B)$ satisfies Assumptions $I(\varphi, \eta)$ and Assumptions $I I(\varphi, \eta, \tilde{d})$ for $\eta>0$, then the $(B S E)$ is globally exactly controllable in $H_{\mathscr{G}}^{s} \cap \widetilde{\mathscr{H}}$ for $s=2+d$ with $d$ from Assumptions $I I(\varphi, \eta, \tilde{d})$ and energetically controllable in $\left(\mu_{k}\right)_{k \in \mathbb{N}^{*}}$.

Proof. From Remark 2.2, the $(B S E)$ is well-posed in $H_{\mathscr{G}}^{s} \cap \widetilde{\mathscr{H}}$ with $s=2+d$ and $d$ from Assumptions $\operatorname{II}(\varphi, \eta, \tilde{d})$. The statement of Theorem 2.3 holds in $\widetilde{\mathscr{H}}$ when the point 1 . is valid, while the validity of [Duc18, Theorem 3.2] in $\widetilde{\mathscr{H}}$ is guaranteed by 2. . The global exact controllability is provided in $H_{\mathscr{G}}^{s} \cap \widetilde{\mathscr{H}}$ and the energetic controllability follows as $\varphi_{k} \in H_{\mathscr{G}}^{s} \cap \widetilde{\mathscr{H}}$ for every $k \in \mathbb{N}^{*}$.

Let $\mathscr{G}$ be a compact quantum graph. By watching the structure of the graph and the boundary conditions of $D(A)$, it is possible to construct some eigenfuctions $\left(\varphi_{k}\right)_{k \in \mathbb{N}^{*}}$ of $A$ corresponding to some eigenvalues $\left(\mu_{k}\right)_{k \in \mathbb{N}^{*}}$. For instance, we consider $\mathscr{G}$ containing one loop $e_{1}$ of length 1 connected to the graph in a vertex $v$. In such case, we point out that the Neumann-Kirchhoff boundary conditions in $v$ valid for a function $\psi \in D(A)$ yield that $\sum_{j \in N(v) \backslash\{1\}} \partial_{x} \psi^{j}(v)+\partial_{x} \psi^{1}(0)-\partial_{x} \psi^{1}(1)=0$.

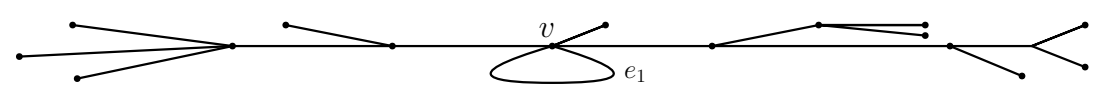

Figure 5: Example of compact graph containing a loop.

We define $\varphi:=\left(\varphi_{k}\right)_{k \in \mathbb{N}^{*}}$ such that $\varphi_{k}=(\sqrt{2} \sin (2 k \pi x), 0, \ldots, 0)$ and the corresponding eigenvalues $\left(\mu_{k}\right)_{k \in \mathbb{N}^{*}}=\left(4 k^{2} \pi^{2}\right)_{k \in \mathbb{N}^{*}} \subseteq\left(\lambda_{k}\right)_{k \in \mathbb{N}^{*}}$, satisfying the gap condition

$$
\inf _{k \in \mathbb{N}^{*}}\left|\mu_{k+1}-\mu_{k}\right|=12 \pi^{2}>0 .
$$

The spectral hypotheses of Theorem 6.1 are guaranteed and the energetic controllability can be ensured by choosing a suitable $B$. In particular, if $(A, B)$ satisfies Assumptions $\mathrm{I}(\varphi, \eta)$ and Assumptions $\operatorname{II}(\varphi, \eta, 0)$ for $\eta>0$, then Theorem 6.1 implies the energetic controllability in $\left(\mu_{k}\right)_{k \in \mathbb{N}^{*}}$. As we will show in the proof of Theorem 6.4, this approach is also valid when $\mathscr{G}$ contains more loops (e.g. Figure 3 ). 
Remark. The idea described above can be adopted when $\mathscr{G}$ contains suitable sub-graphs denoted "uniform chains". A uniform chain is a sequence of edges of equal length $L$ connecting $M \in \mathbb{N}^{*}$ vertices $\left\{v_{j}\right\}_{j \leq M}$ such that $v_{2}, \ldots, v_{M-1} \in V_{i}$ when $M \geq 3$. Moreover, one of the following conditions holds: either $v_{1}, v_{M} \in V_{e}$ are equipped with $(\mathcal{D})$, or $v_{1}=v_{M}$ belong to $V_{i}$, or $M \in\{2,3\}$ and $v_{1}, v_{M} \in V_{e}$ are equipped with $(\mathcal{N})$.

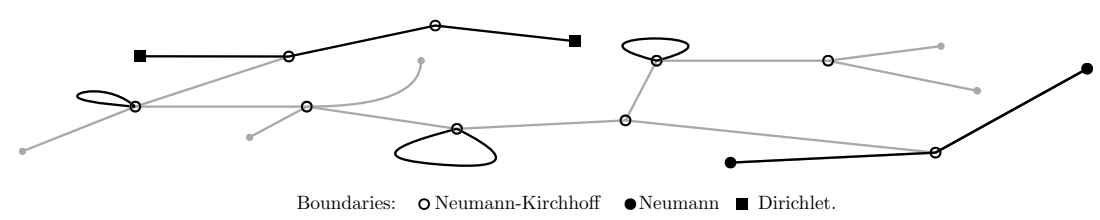

Figure 6: The figure underlines the uniform chains in a compact graph.

Let $\mathscr{G}$ contain $\tilde{N} \in \mathbb{N}^{*}$ uniform chains $\left\{\widetilde{\mathscr{G}}_{j}\right\}_{j \leq \widetilde{N}}$, composed by edges of lengths $\left\{L_{j}\right\}_{j \leq \widetilde{N}} \in \mathcal{A} \mathcal{L}(\widetilde{N})$. Let $I_{1} \subseteq\{1, \ldots, \tilde{N}\}$ and $I_{2} \subseteq\{1, \ldots, \tilde{N}\} \backslash I_{1}$ be respectively the sets of indices $j$ such that the external vertices of $\widetilde{\mathscr{G}}_{j}$ are equipped with $(\mathcal{N})$ and $(\mathcal{D})$, while $I_{3}:=\{1, \ldots, \tilde{N}\} \backslash\left(I_{1} \cup I_{2}\right)$. We consider the eigenvalues $\left(\mu_{k}\right)_{k \in \mathbb{N}^{*}}$ obtained by reordering

$$
\left(\frac{(2 k-1)^{2} \pi^{2}}{4 L_{j}^{2}}\right)_{\substack{k, j \in \mathbb{N}^{*} \\ j \in I_{1}}} \cup\left(\frac{k^{2} \pi^{2}}{L_{j}^{2}}\right)_{\substack{k, j \in \mathbb{N}^{*} \\ j \in I_{2}}} \cup\left(\frac{4 k^{2} \pi^{2}}{L_{j}^{2}}\right)_{\substack{k, j \in \mathbb{N}^{*} \\ j \in I_{3}}}
$$

As in the proof of [Duc18,Lemma 2.6], the Roth's Theorem [Duc18, Proposition A.1] ensures that, if $\left\{L_{j}\right\}_{j \leq \widetilde{N}} \in \mathcal{A L}(\widetilde{N})$, then for every $\epsilon>0$, there exists $C>0$ so that

$$
\left|\mu_{k+1}-\mu_{k}\right| \geq C k^{-\epsilon}, \quad \forall k \in \mathbb{N}^{*} \text {. }
$$

Thus, the spectral hypotheses of Theorem 6.1 are guaranteed and the energetic controllability can be ensured by choosing a suitable control operator $B$. If $(A, B)$ satisfies Assumptions $I(\varphi, \eta)$ and Assumptions $I I(\varphi, \eta, \epsilon)$ with $\eta>0$, then Theorem 6.1 implies the energetic controllability in $\left(\mu_{k}\right)_{k \in \mathbb{N}^{*}}$.

\subsection{Proof of Theorem 1.6 and some applications of Theorem 6.1}

Proof of Theorem 1.6. Let us assume $N=3$. The $(\mathcal{D})$ conditions to the external vertices $V_{e}$ imply $\phi_{k}=$ $\left(a_{k}^{1} \sin \left(\sqrt{\mu_{k}} x\right), a_{k}^{2} \sin \left(\sqrt{\mu_{k}} x\right), a_{k}^{3} \sin \left(\sqrt{\mu_{k}} x\right)\right)$ with suitable $\left(a_{k}^{1}, a_{k}^{2}, a_{k}^{3}\right) \in \mathbb{C}^{3}$. From the $(\mathcal{N} \mathcal{K})$ in $v \in V_{i}$, there follow $\sum_{l \leq 3} a_{k}^{l} \cos \left(\sqrt{\mu_{k}} L\right)=0$ and $a_{k}^{m} \sin \left(\sqrt{\mu_{k}} L\right)=c \in \mathbb{R}$ for every $m \leq 3$. When $c \neq 0$, we have the eigenvalues $\left(\frac{(2 k-1)^{2} \pi^{2}}{4 L^{2}}\right)_{k \in \mathbb{N}^{*}}$ corresponding to the eigenfunctions $\left(g_{k}\right)_{k \in \mathbb{N}^{*}}$ so that

$$
g_{k}=\left(\sqrt{\frac{2}{3 L}} \sin \left(\frac{(2 k-1) \pi}{2 L} x\right), \sqrt{\frac{2}{3 L}} \sin \left(\frac{(2 k-1) \pi}{2 L} x\right), \sqrt{\frac{2}{3 L}} \sin \left(\frac{(2 k-1) \pi}{2 L} x\right)\right), \quad \forall k \in \mathbb{N}^{*} .
$$

When $c=0$, we obtain the eigenvalues $\left(\frac{k^{2} \pi^{2}}{L^{2}}\right)_{k \in \mathbb{N}^{*}}$ of multiplicity two that we associate to the couple of sequences of eigenfunctions $\left(f_{k}^{1}\right)_{k \in \mathbb{N}^{*}}$ and $\left(f_{k}^{2}\right)_{k \in \mathbb{N}^{*}}$ such that, for every $k \in \mathbb{N}^{*}$,

$$
\begin{gathered}
f_{k}^{1}:=\left(-\sqrt{\frac{4}{3 L}} \sin \left(\frac{k \pi}{L} x\right), \sqrt{\frac{1}{3 L}} \sin \left(\frac{k \pi}{L} x\right), \sqrt{\frac{1}{3 L}} \sin \left(\frac{k \pi}{L} x\right)\right), \\
f_{k}^{2}:=\left(0,-\sqrt{\frac{1}{L}} \sin \left(\frac{k \pi}{L} x\right), \sqrt{\frac{1}{L}} \sin \left(\frac{k \pi}{L} x\right)\right) .
\end{gathered}
$$

Moreover, $\left(f_{k}^{1}\right)_{k \in \mathbb{N}^{*}} \cup\left(f_{k}^{2}\right)_{k \in \mathbb{N}^{*}} \cup\left(g_{k}\right)_{k \in \mathbb{N}^{*}}$ is a Hilbert basis of $\mathscr{H}$ and $\left(\frac{k^{2} \pi^{2}}{L^{2}}\right)_{k \in \mathbb{N}^{*}} \cup\left(\frac{(2 k-1)^{2} \pi^{2}}{4 L^{2}}\right)_{k \in \mathbb{N}^{*}}$ are the eigenvalues of $A$ (not considering their multiplicity).

Validation of Assumptions $\mathbf{I}(\varphi, \mathbf{1})$. We reorder $\left(f_{k}^{1}\right)_{k \in \mathbb{N}^{*}} \cup\left(g_{k}\right)_{k \in \mathbb{N}^{*}}$ in $\varphi=\left(\varphi_{k}\right)_{k \in \mathbb{N}^{*}}$. The point 1. of Assumptions $\mathrm{I}(\varphi, 1)$ is verified since there exists $C_{1}, C_{2}>0$ such that

$$
\left|\left\langle\varphi_{1}, B \varphi_{k}\right\rangle_{L^{2}}\right| \geq \frac{C_{1} \sqrt{\mu_{k}} \sqrt{\mu_{1}}}{\left(\mu_{k}-\mu_{1}\right)^{2}} \geq \frac{C_{2}}{k^{3}}, \quad \forall k \in \mathbb{N}^{*}
$$

Subsequently, there exist $C_{3}, C_{4}>0$ so that $B_{k, k}:=\left\langle\varphi_{k}, B \varphi_{k}\right\rangle_{L^{2}}=C_{3}+C_{4} k^{-2}$ for every $k \in \mathbb{N}^{*}$ and $\mu_{k}=\frac{\pi^{2} k^{2}}{4 L^{2}}$. Now, if $\mu_{j}-\mu_{k}-\mu_{l}+\mu_{m}=\frac{\pi^{2}}{4 L^{2}}\left(j^{2}-k^{2}-l^{2}+m^{2}\right)=0$ with $(k, j),(m, n) \in I$ and $(k, j) \neq(m, n)$, then

$$
B_{j, j}-B_{k, k}-B_{l, l}+B_{m, m}=C_{4}\left(j^{-2}-k^{-2}-l^{-2}+m^{-2}\right) \neq 0,
$$


which implies the point 2. of Assumptions $\mathrm{I}(\varphi, 1)$.

Validation of Assumptions $\mathbf{I I}(\varphi, \mathbf{1}, \mathbf{0})$ and conclusion. The operator $B$ stabilizes the spaces $H^{m}$ with $m>0$ and $\overline{\operatorname{span}\left\{\varphi_{k}: k \in \mathbb{N}^{*}\right\}} L^{2} \cap H_{\mathscr{G}}^{2}$, ensuring the point 1. of Assumptions $\operatorname{II}(\varphi, 1,0)$. Since

$$
\inf _{j, k \in \mathbb{N}^{*}}\left|\mu_{k}-\mu_{j}\right|=\frac{\pi^{2}}{4 L^{2}}
$$

the point 2. of Theorem 6.1 holds and the global exact controllability is proved in $H_{\mathscr{G}}^{3} \cap \widetilde{\mathscr{H}}$. As $\varphi_{k} \in H_{\mathscr{G} \cap}^{3} \cap \widetilde{\mathscr{H}}$ for every $k \in \mathbb{N}^{*}$, the energetic controllability follows in $\left(\frac{k^{2} \pi^{2}}{4 L^{2}}\right)_{k \in \mathbb{N}^{*}}$.

When $N>3$, the spectrum contains simple eigenvalues relative to some eigenfunctions $\left(g_{k}\right)_{k \in \mathbb{N}^{*}}$ and multiple eigenvalues, each one corresponding to $N-1$ eigenfunctions $\left\{f_{k ; j}\right\}_{l<N-1}$ with $k \in \mathbb{N}^{*}$. For each $k \in \mathbb{N}^{*}$, we construct $\left\{f_{k ; j}\right\}_{l \leq N-1}$ such that only the functions $\left\{f_{k ; j}\right\}_{l \leq N-2}$ vanish in $e_{1}$. We reorder $\left(f_{k ; N-1}\right)_{k \in \mathbb{N}^{*}} \cup\left(g_{k}\right)_{k \in \mathbb{N}^{*}}$ in $\varphi=\left(\varphi_{k}\right)_{k \in \mathbb{N}^{*}}$ and the proof is achieved as done for $N=3$.

Theorem 6.2. Let $\mathscr{G}$ be a star graph equipped with $(\mathcal{D} / \mathcal{N})$. Let $\mathscr{G}$ contain two edges $e_{1}$ and $e_{2}$ of length 1 and connected to two external vertices both equipped with $(\mathcal{D})$. Let $B$ be such that

$$
B \psi=\left(x^{2}\left(\psi^{1}(x)-\psi^{2}(x)\right), x^{2}\left(\psi^{2}(x)-\psi^{1}(x)\right), 0, \ldots, 0\right), \quad \forall \psi \in \mathscr{H} .
$$

There exists $\left(\varphi_{k}\right)_{k \in \mathbb{N}^{*}} \subset\left(\phi_{k}\right)_{k \in \mathbb{N}^{*}}$ such that the $(B S E)$ is globally exactly controllable in $H_{\mathscr{G}}^{3} \cap \widetilde{\mathscr{H}}$ and energetically controllable in $\left(k^{2} \pi^{2}\right)_{k \in \mathbb{N}^{*}}$.

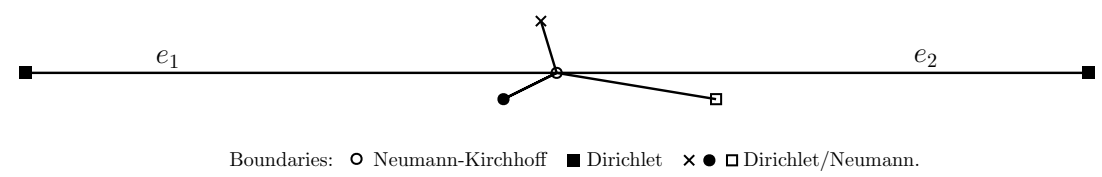

Figure 7: Example of star graph described by Theorem 6.2 with $N=5$.

Proof. Let $\left(\mu_{k}\right)_{k \in \mathbb{N}^{*}}$ and $\left(\varphi_{k}\right)_{k \in \mathbb{N}^{*}}$ be so that $\mu_{k}=k^{2} \pi^{2}, \varphi_{k}^{1}=-\varphi_{k}^{2}=\sin (k \pi x)$ and $\varphi_{k}^{l}=0$ with $k \in \mathbb{N}^{*}$ and $3 \leq l \leq N$. The claim follows from the point 2. of Theorem 6.1 with $\tilde{d}=0$ as Theorem 1.6.

Theorem 6.3. Let $\mathscr{G}$ be a star graph equipped with $(\mathcal{D})$ and composed by $\frac{N}{2}$ couples of edges $\left\{e_{2 j-1}, e_{2 j}\right\}_{j \leq \frac{N}{2}}$ of lengths $\left\{L_{j}\right\}_{j \leq \frac{N}{2}} \in \mathcal{A L}\left(\frac{N}{2}\right)$ with $N \in 2 \mathbb{N}^{*}$. Let $B$ be such that $B \psi=\left((B \psi)^{1}, \ldots,(B \psi)^{N}\right)$ for $\psi \in \mathscr{H}$ and

$$
(B \psi)^{2 j}=-(B \psi)^{2 j-1}=\sum_{l=1}^{N / 2} \frac{L_{l}^{3 / 2}}{L_{j}^{3 / 2}} x^{2}\left(\psi^{2 l}\left(\frac{L_{l}}{L_{j}} x\right)-\psi^{2 l-1}\left(\frac{L_{l}}{L_{j}} x\right)\right), \quad \forall j \leq \frac{N}{2} .
$$

There exists $\mathcal{C} \subset\left(\mathbb{R}^{+}\right)^{N}$ countable so that, for every $\left\{L_{j}\right\}_{j \leq N} \in \mathcal{A L}(N) \backslash \mathcal{C}$, there exists $\left(\varphi_{k}\right)_{k \in \mathbb{N}^{*}} \subseteq$ $\left(\phi_{k}\right)_{k \in \mathbb{N}^{*}}$ such that $(B S E)$ is globally exactly controllable in $H_{\mathscr{G}}^{3+\epsilon} \cap \widetilde{\mathscr{H}}$ with $\epsilon>0$ and energetically controllable in $\left(\frac{k^{2} \pi^{2}}{L_{j}^{2}}\right)_{\substack{k, j \in \mathbb{N}^{*} \\ j \leq N / 2}}$.

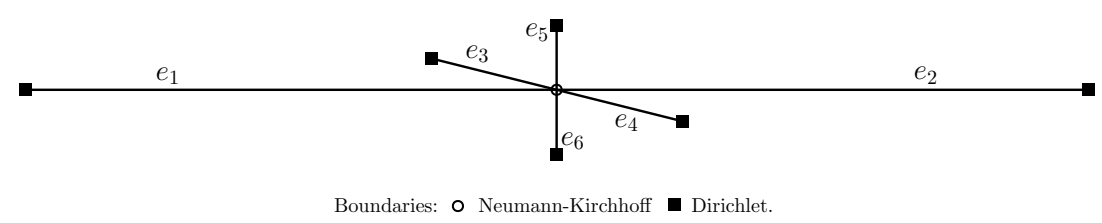

Figure 8: Example of star graph described by Theorem 6.3 with $N=6$.

Proof. Let $\left(\mu_{k}\right)_{k \in \mathbb{N}^{*}} \subset\left(\lambda_{k}\right)_{k \in \mathbb{N}^{*}}$ be eigenvalues obtained by reordering $\left(\frac{k^{2} \pi^{2}}{L_{j}^{2}}\right)_{k \in \mathbb{N}^{*}}$ for every $j \leq N / 2$ and $\left(\varphi_{k}\right)_{k \in \mathbb{N}^{*}}$ be an orthonormal system of $\mathscr{H}$ made by corresponding eigenfunctions. For $k \in \mathbb{N}^{*}$, there exist $m(k) \in \mathbb{N}^{*}$ and $l(k) \leq N / 2$ so that $\varphi_{k}^{n} \equiv 0$ for $n \neq 2 l(k), 2 l(k)-1$ and

$$
\mu_{k}=\frac{m(k)^{2} \pi^{2}}{L_{l(k)}^{2}}, \quad \varphi_{k}^{2 l(k)-1}(x)=-\varphi_{k}^{2 l(k)}(x)=\sqrt{\frac{1}{L_{l(k)}}} \sin \left(\sqrt{\mu_{k}} x\right) .
$$


Let $[r]$ be the entire part of $r \in \mathbb{R}^{+}$. For $k \in \mathbb{N}^{*}$ and $C=4 \min _{l \leq N} L_{l}$, we have

$$
\begin{aligned}
& \left|\left\langle\varphi_{1}, B \varphi_{k}\right\rangle_{L^{2}}\right|=\left|\sum_{l=1}^{N}\left\langle\varphi_{k}^{l}(x), \sum_{n=1}^{N / 2} \frac{L_{n}^{\frac{3}{2}} x^{2}}{L_{[(l+1) / 2]}^{\frac{3}{2}}}\left(\varphi_{1}^{2 n-1}\left(\frac{L_{n}}{L_{[(l+1) / 2]}} x\right)-\varphi_{1}^{2 n}\left(\frac{L_{n}}{L_{[(l+1) / 2]}} x\right)\right)\right\rangle_{L^{2}\left(e_{l}\right)}\right| \\
& =\left|\int_{0}^{L_{l(k)}} \frac{4 x^{2} L_{l(1)}^{\frac{3}{2}}}{L_{l(k)}^{\frac{3}{2}}} \sin \left(\frac{m(1) \pi x}{L_{l(k)}}\right) \sin \left(\frac{m(k) \pi x}{L_{l(k)}}\right) d x\right| \geq C\left|\int_{0}^{1} x^{2} \sin (m(1) \pi x) \sin (m(k) \pi x) d x\right| .
\end{aligned}
$$

Assumptions $\mathrm{I}(\varphi, 1)$ and Assumptions $\operatorname{II}(\varphi, 1, \epsilon)$ with $\epsilon \in\left(0, \frac{1}{2}\right)$ hold as in the proofs of Theorem 1.4 and Theorem 1.6. We consider the techniques adopted in the proof of [Duc18, Lemma 2.6] which are due to the Roth's Theorem [Duc18, Proposition A.1]. For every $\epsilon>0$, there exists $C>0$ so that

$$
\left|\mu_{k+1}-\mu_{k}\right| \geq C k^{-\epsilon}, \quad \forall k \in \mathbb{N}^{*} .
$$

The claim follows from the hypotheses 2. of Theorem 6.1 with $\tilde{d}=\epsilon>0$.

Theorem 6.4. Let $\mathscr{G}$ be a compact quantum graph. Let the first $\widetilde{N} \leq N$ edges $\left\{e_{j}\right\}_{j \leq \widetilde{N}}$ of the graph be loops of lengths $\left\{L_{j}\right\}_{j \leq \widetilde{N}}$ (e.g Figure 3$)$. For $\psi=\left(\psi^{1}, \ldots \psi^{N}\right)$, let $B$ be such that

$$
(B \psi)^{l}=\sum_{j \leq \widetilde{N}} \frac{L_{j}^{3 / 2}}{L_{l}^{3 / 2}} x^{2}\left(\frac{x}{L_{l}}-1\right) \psi^{j}\left(\frac{L_{j}}{L_{l}} x\right), \quad(B \psi)^{m} \equiv 0, \quad \forall l \leq \widetilde{N}, \quad \tilde{N}<m \leq N .
$$

There exists $\mathcal{C} \subset\left(\mathbb{R}^{+}\right)^{\widetilde{N}}$ countable so that, if $\left\{L_{j}\right\}_{j \leq \widetilde{N}} \in \mathcal{A L}(\widetilde{N}) \backslash \mathcal{C}$, then there exists $\left(\varphi_{k}\right)_{k \in \mathbb{N}^{*}} \subseteq\left(\phi_{k}\right)_{k \in \mathbb{N}^{*}}$ such that $(B S E)$ is globally exactly controllable in $H_{\mathscr{G}}^{3+\epsilon} \cup \widetilde{\mathscr{H}}$ with $\epsilon>0$ and energetically controllable in $\left(\frac{k^{2} \pi^{2}}{L_{j}^{2}}\right)_{\substack{k, j \in \mathbb{N}^{*} \\ j \leq \mathbb{N}}}$

Proof. Let $\left(\varphi_{k}\right)_{k \in \mathbb{N}^{*}}$ be such that, for each $k \in \mathbb{N}^{*}$, there exist $m(k) \in \mathbb{N}^{*}$ and $l(k) \leq \widetilde{N}$ such that $\mu_{k}=\frac{4 m(k)^{2} \pi^{2}}{L_{l(k)}^{2}}, \varphi_{k}^{l(k)}(x)=\sqrt{\frac{2}{L_{l(k)}}} \sin \left(\sqrt{\mu_{k}} x\right)$ and $\varphi_{k}^{n} \equiv 0$ for every $n \neq l(k)$ and $n \leq N$. Now, $\left(\varphi_{k}\right)_{k \in \mathbb{N}^{*}}$ is an orthonormal system made by eigenfunctions of $A$ and the claim yields as Theorem 6.3.

Acknowledgments. The author would like to thank the referees for the constructive comments which improved the quality of redaction. He is also grateful to Olivier Glass and Nabile Boussaïd for having carefully reviewed this work. Finally, he thanks Kaïs Ammari for suggesting him the problem and the colleagues Andrea Piras, Riccardo Adami, Enrico Serra and Paolo Tilli for the fruitful conversations.

\section{A Appendix: Global approximate controllability}

In the current appendix, for the sake of completeness, we propose the global approximate controllability result provided in [Duc18, Section 5.2]. The outcome is adopted in the proof of Theorem 2.3.

Definition A.1. The (BSE) is said to be globally approximately controllable in $H_{\mathscr{G}}^{s}$ with $s>0$ when, for every $\psi \in H_{\mathscr{G}}^{s}, \widehat{\Gamma} \in U(\mathscr{H})$ such that $\widehat{\Gamma} \psi \in H_{\mathscr{G}}^{s}$ and $\varepsilon>0$, there exist $T>0$ and $u \in L^{2}((0, T), \mathbb{R})$ such that $\left\|\widehat{\Gamma} \psi-\Gamma_{T}^{u} \psi\right\|_{(s)}<\varepsilon$.

Proposition A.2. Let $(A, B)$ satisfy Assumptions $I(\eta)$ and Assumptions $I I(\eta, \tilde{d})$ for $\eta>0$ and $\widetilde{d} \geq 0$. The (BSE) is globally approximately controllable in $H_{\mathscr{G}}^{s}$ for $s=2+d$ with $d$ from Assumptions $I I(\eta, \tilde{\tilde{d}})$.

Proof. In the point $\mathbf{1})$ of the proof, we suppose that $(A, B)$ admits a non-degenerate chain of connectedness (see [BdCC13, Definition 3]). We treat the general case in the point 2) .

1) (a) Preliminaries. Let $\pi_{m}$ be the orthogonal projector onto $\mathscr{H}_{m}:=\operatorname{span}\left\{\phi_{j}: j \leq m\right\}$ with $m \in \mathbb{N}^{*}$. Up to reordering $\left(\phi_{k}\right)_{k \in \mathbb{N}^{*}}$, the couples $\left(\pi_{m} A \pi_{m}, \pi_{m} B \pi_{m}\right)$ for $m \in \mathbb{N}^{*}$ admit non-degenerate chains of connectedness in $\mathscr{H}_{m}$. Let $\|\cdot\|_{B V(T)}=\|\cdot\|_{B V((0, T), \mathbb{R})}$ and $\|\cdot\|\left\|_{(s)}:=\right\|\|\|_{L\left(H_{g}^{s}, H_{g g}^{s}\right)}$ for $s>0$. For $N \in \mathbb{N}^{*}$, we denote $S U\left(\mathscr{H}_{N}\right)=\left\{\Gamma \in U\left(\mathscr{H}_{N}\right):\left(\left\langle\phi_{j}, \Gamma \phi_{k}\right\rangle_{L^{2}}\right)_{j, k \leq N} \in S U(N)\right\}$.

Claim. $\forall \widehat{\Gamma} \in U(\mathscr{H}), \forall \varepsilon>0, \exists N_{1} \in \mathbb{N}^{*}, \widetilde{\Gamma}_{N_{1}} \in U(\mathscr{H}): \pi_{N_{1}} \widetilde{\Gamma}_{N_{1}} \pi_{N_{1}} \in S U\left(\mathscr{H}_{N_{1}}\right)$,

$$
\left\|\widetilde{\Gamma}_{N_{1}} \phi_{1}-\widehat{\Gamma} \phi_{1}\right\|_{L^{2}}<\varepsilon \text {. }
$$


Let $N_{1} \in \mathbb{N}^{*}$ and $\widetilde{\phi}_{1}:=\left\|\pi_{N_{1}} \widehat{\Gamma} \phi_{1}\right\|_{L^{2}}^{-1} \pi_{N_{1}} \widehat{\Gamma} \phi_{1}$. We define $\left(\widetilde{\phi}_{j}\right)_{2 \leq j \leq N_{1}}$ such that $\left(\widetilde{\phi}_{j}\right)_{j \leq N_{1}}$ is an orthonormal basis of $\mathscr{H}_{N_{1}}$ and we complete it to $\left(\widetilde{\phi}_{j}\right)_{j \in \mathbb{N}^{*}}$, a Hilbert basis of $\mathscr{H}$. The operator $\widetilde{\Gamma}_{N_{1}}$ is the unitary map such that $\widetilde{\Gamma}_{N_{1}} \phi_{j}=\widetilde{\phi}_{j}$ for every $j \in \mathbb{N}^{*}$. The provided definition implies $\lim _{N_{1} \rightarrow \infty}\left\|\widetilde{\Gamma}_{N_{1}} \phi_{1}-\widehat{\Gamma} \phi_{1}\right\|_{L^{2}}=0$. Thus, for every $\varepsilon>0$, there exists $N_{1} \in \mathbb{N}^{*}$ large enough satisfying the claim.

1) (b) Finite dimensional controllability. Let $M_{a d}$ be the set of $(j, k) \in\left\{1, \ldots, N_{1}\right\}^{2}$ such that $B_{j, k}:=\left\langle\phi_{j}, B \phi_{k}\right\rangle_{L^{2}} \neq 0$ and $\left|\lambda_{j}-\lambda_{k}\right|=\left|\lambda_{m}-\lambda_{l}\right|$ with $m, l \in \mathbb{N}^{*}$ implies $\{j, k\}=\{m, l\}$ for $B_{m, l}=0$. For every $(j, k) \in\left\{1, \ldots, N_{1}\right\}^{2}$ and $\theta \in[0,2 \pi)$, we define $E_{j, k}^{\theta}$ the $N_{1} \times N_{1}$ matrix with elements $\left(E_{j, k}^{\theta}\right)_{l, m}=0$, $\left(E_{j, k}^{\theta}\right)_{j, k}=e^{i \theta}$ and $\left(E_{j, k}^{\theta}\right)_{k, j}=-e^{-i \theta}$ for $(l, m) \in\left\{1, \ldots, N_{1}\right\}^{2} \backslash\{(j, k),(k, j)\}$. Let $E_{a d}=\left\{E_{j, k}^{\theta}:(j, k) \in\right.$ $\left.M_{a d}, \theta \in[0,2 \pi)\right\}$. Let $L_{1}:=E_{a d}$. We define by iteration $L_{m}:=\left[E_{a d}, L_{m-1}\right]+L_{m-1}$ with $m \in \mathbb{N}^{*} \backslash\{1\}$ and there exists $\widetilde{m} \in \mathbb{N}^{*}$ such that $L_{\widetilde{m}}=L_{\widetilde{m}+1}$ as $E_{a d}$ is composed by $N_{1} \times N_{1}$ matrices. We denote $\operatorname{Lie}\left(E_{a d}\right)=L_{\widetilde{m}}$. Fixed $v$ a piecewise constant control taking value in $E_{a d}$ and $\tau>0$, we introduce the control system on $S U\left(N_{1}\right)$

$$
\left\{\begin{array}{l}
\dot{x}(t)=x(t) v(t), \quad t \in(0, \tau), \\
x(0)=I d_{S U\left(N_{1}\right)} .
\end{array}\right.
$$

Claim. (37) is controllable, i.e. for $R \in S U\left(N_{1}\right)$, there exist $p \in \mathbb{N}^{*}, M_{1}, \ldots, M_{p} \in E_{a d}, \alpha_{1}, \ldots, \alpha_{p} \in$ $\mathbb{R}^{+}$such that $R=e^{\alpha_{1} M_{1}} \circ \ldots \circ e^{\alpha_{p} M_{p}}$.

For every $(j, k) \in\left\{1, \ldots, N_{1}\right\}^{2}$, we define the $N_{1} \times N_{1}$ matrices $R_{j, k}, C_{j, k}$ and $D_{j}$ as follow. For $(l, m) \in$ $\left\{1, \ldots, N_{1}\right\}^{2} \backslash\{(j, k),(k, j)\}$, we have $\left(R_{j, k}\right)_{l, m}=0$ and $\left(R_{j, k}\right)_{j, k}=-\left(R_{j, k}\right)_{k, j}=1$, while $\left(C_{j, k}\right)_{l, m}=0$ and $\left(C_{j, k}\right)_{j, k}=\left(C_{j, k}\right)_{k, j}=i$. Moreover, for $(l, m) \in\left\{1, \ldots, N_{1}\right\}^{2} \backslash\{(1,1),(j, j)\},\left(D_{j}\right)_{l, m}=0$ and $\left(D_{j}\right)_{1,1}=$ $-\left(D_{j}\right)_{j, j}=i$. We consider the basis of $s u\left(N_{1}\right)$, the Lie algebra of $S U\left(N_{1}\right)$,

$$
\left\{R_{j, k}\right\}_{j, k \leq N_{1}} \cup\left\{C_{j, k}\right\}_{j, k \leq N_{1}} \cup\left\{D_{j}\right\}_{j \leq N_{1}} .
$$

Thanks to [Sac00, Theorem 6.1], the controllability of (37) is equivalent to prove that $\operatorname{Lie}\left(E_{a d}\right) \supseteq s u\left(N_{1}\right)$. The claim is valid since it is possible to obtain the matrices $R_{j, k}, C_{j, k}$ and $D_{j}$ for every $j, k \leq N_{1}$ by iterated Lie brackets of elements in $E_{a d}$ as follows.

- For every $(j, k) \in M_{a d}$, we have $R_{j, k}=E_{j, k}^{0}$ and $C_{j, k}=E_{j, k}^{\frac{\pi}{2}}$. For every $(j, k) \notin M_{a d}$ such that there exists $j_{1} \leq N_{1}$ so that $\left(j, j_{1}\right),\left(j_{1}, k\right) \in M_{a d}$, we have $R_{j, k}=\left[E_{j, j_{1}}^{0}, E_{j_{1}, k}^{0}\right]$ and $C_{j, k}=\left[E_{j, j_{1}}^{0}, E_{j_{1}, k}^{\frac{\pi}{2}}\right]$.

- If $(1, j) \in M_{a d}$, then $2 D_{j}=\left[E_{1, j}^{0}, E_{1, j}^{\frac{\pi}{2}}\right]$, while if $(1, j) \notin M_{a d}$ and there exists $j_{1} \leq N_{1}$ such that $\left(1, j_{1}\right),\left(j_{1}, j\right) \in M_{a d}$, then $-2 D_{j}=\left[\left[E_{1, j_{1}}^{\frac{\pi}{2}}, E_{j_{1}, j}^{\frac{\pi}{2}}\right],\left[E_{1, j_{1}}^{0}, E_{j_{1}, j}^{\frac{\pi}{2}}\right]\right]$.

- For every $(j, k) \notin M_{a d}$, there exist $m \leq N_{1}$ and $\left\{j_{l}\right\}_{l \leq m}$ such that $\left(j, j_{1}\right), \ldots,\left(j_{m}, k\right) \in M_{a d}$. We call $S=\left\{\left(j, j_{1}\right), \ldots,\left(j_{m}, k\right)\right\}$. By repeating the previous point, we can generate each $R_{j, k}, C_{j, k}$ and $D_{j}$ with $j, k \in\left\{1, \ldots, N_{1}\right\}$ by iterating Lie brackets of $E_{l, m}^{\theta}$ for $(l, m) \in S$ and $\theta \in[0,2 \pi)$.

1) (c) Finite dimensional estimates. The previous claim and the fact that $\left(\left\langle\phi_{j}, \widetilde{\Gamma}_{N_{1}} \phi_{k}\right\rangle_{L^{2}}\right)_{j, k \leq N_{1}} \in$ $S U\left(N_{1}\right)$ ensure the existence of $p \in \mathbb{N}^{*}, M_{1}, \ldots, M_{p} \in E_{a d}$ and $\alpha_{1}, \ldots, \alpha_{p} \in \mathbb{R}^{+}$such that

$$
\left(\left\langle\phi_{j}, \widetilde{\Gamma}_{N_{1}} \phi_{k}\right\rangle_{L^{2}}\right)_{j, k \leq N_{1}}=e^{\alpha_{1} M_{1}} \circ \ldots \circ e^{\alpha_{p} M_{p}} .
$$

For every $l \leq p$, we call $\widehat{\Gamma}_{l}$ the operator in $S U\left(\mathscr{H}_{N_{1}}\right)$ such that $\left(\left\langle\phi_{j}, \widehat{\Gamma}_{l} \phi_{k}\right\rangle\right)_{j, k \leq N_{1}}=e^{\alpha_{l} M_{l}}$. The identity (38) yields

$$
\pi_{N_{1}} \widetilde{\Gamma}_{N_{1}} \pi_{N_{1}}=\widehat{\Gamma}_{1} \circ \ldots \circ \widehat{\Gamma}_{p}
$$

Claim. For every $l \leq p$, there exist $\left\{T_{n}^{l}\right\}_{l \in \mathbb{N}^{*}} \subset \mathbb{R}^{+}$and $\left\{u_{n}^{l}\right\}_{n \in \mathbb{N}^{*}}$ such that $u_{n}^{l} \in L^{2}\left(\left(0, T_{n}^{l}\right), \mathbb{R}\right)$ for every $n \in \mathbb{N}^{*}$ and

$$
\begin{gathered}
\lim _{n \rightarrow \infty}\left\|\Gamma_{T_{n}^{l}}^{u_{n}^{l}} \phi_{k}-\widehat{\Gamma}_{l} \phi_{k}\right\|_{L^{2}}=0, \quad \forall k \leq N_{1}, \\
\sup _{n \in \mathbb{N}^{*}}\left\|u_{n}^{l}\right\|_{B V\left(T_{n}^{l}\right)<\infty,} \quad \sup _{n \in \mathbb{N}^{*}}\left\|u_{n}^{l}\right\|_{L^{\infty}\left(\left(0, T_{n}^{l}\right), \mathbb{R}\right)}<\infty, \quad \sup _{n \in \mathbb{N}^{*}} T_{n}^{l}\left\|u_{n}^{l}\right\|_{L^{\infty}\left(\left(0, T_{n}^{l}\right), \mathbb{R}\right)}<\infty .
\end{gathered}
$$


We consider the results developed in [Cha12, Section 3.1 \& Section 3.2] by Chambrion and leading to [Cha12, Proposition 6] since $(A, B)$ admits a non-degenerate chain of connectedness ([BdCC13, Definition $3])$. Each $\widehat{\Gamma}_{l}$ is a rotation in a two dimensional space for every $l \in\{1, \ldots, p\}$ and this work explicits $\left\{T_{n}^{l}\right\}_{n \in \mathbb{N}^{*}} \subset \mathbb{R}^{+}$and $\left\{u_{n}^{l}\right\}_{n \in \mathbb{N}^{*}}$ satisfying (41) such that $u_{n}^{l} \in L^{2}\left(\left(0, T_{n}^{l}\right), \mathbb{R}\right)$ for every $n \in \mathbb{N}^{*}$ and

$$
\lim _{n \rightarrow \infty}\left\|\pi_{N_{1}} \Gamma_{T_{n}^{l}}^{u_{n}^{l}} \phi_{k}-\widehat{\Gamma}_{l} \phi_{k}\right\|_{L^{2}}=0, \quad \forall k \leq N_{1}
$$

Now, $\lim _{n \rightarrow \infty}\left\|\pi_{N_{1}} \Gamma_{T_{n}^{l}}^{u_{n}^{l}} \phi_{k}\right\|_{L^{2}}=1$ since $\widehat{\Gamma}_{l} \in S U\left(\mathscr{H}_{N_{1}}\right)$. Thus, $\lim _{n \rightarrow \infty}\left\|\left(1-\pi_{N_{1}}\right) \Gamma_{T_{n}^{l}}^{u_{n}^{l}} \phi_{k}\right\|_{L^{2}}=0$ and

$$
\lim _{n \rightarrow \infty}\left\|\Gamma_{T_{n}^{l}}^{u_{n}^{l}} \phi_{k}-\widehat{\Gamma}_{l} \phi_{k}\right\|_{L^{2}} \leq \lim _{n \rightarrow \infty}\left(\left\|\pi_{N_{1}} \Gamma_{T_{n}^{l}}^{u_{n}^{l}} \phi_{k}-\widehat{\Gamma}_{l} \phi_{k}\right\|_{L^{2}}+\left\|\left(1-\pi_{N_{1}}\right) \Gamma_{T_{n}^{l}}^{u_{n}^{l}} \phi_{k}\right\|_{L^{2}}\right)=0 .
$$

1) (d) Infinite dimensional estimates.

Claim. Let $\widehat{\Gamma} \in U(\mathscr{H})$. There exist $K_{1}, K_{2}, K_{3}>0$ such that for every $\varepsilon>0$, there exist $T>0$ and $u \in L^{2}((0, T), \mathbb{R})$ such that $\left\|\Gamma_{T}^{u} \phi_{1}-\widehat{\Gamma} \phi_{1}\right\|_{L^{2}}<\varepsilon$ and

$$
\|u\|_{B V(T)} \leq K_{1}, \quad\|u\|_{L^{\infty}((0, T), \mathbb{R})} \leq K_{2}, \quad T\|u\|_{L^{\infty}((0, T), \mathbb{R})} \leq K_{3} .
$$

Let us assume that 1) (c) be valid with $p=2$. Although, the following result is valid for any $p \in \mathbb{N}^{*}$. As $\widehat{\Gamma}_{2} \in S U\left(\mathscr{H}_{N_{1}}\right)$, there exist $l \leq N_{1}$ and $\alpha \in \mathbb{C}$ such that $|\alpha|=1$ and $\widehat{\Gamma}_{2} \phi_{1}=\alpha \phi_{l}$. Thanks to (40), there exists $n \in \mathbb{N}^{*}$ large enough such that,

$$
\left\|\Gamma_{T_{n}^{1}}^{u_{n}^{1}} \Gamma_{T_{n}^{2}}^{u_{2}^{2}} \phi_{1}-\widehat{\Gamma}_{1} \widehat{\Gamma}_{2} \phi_{1}\right\|_{L^{2}} \leq\left\|\mid \Gamma_{T_{n}^{1}}^{u_{n}^{1}}\right\|\left\|\Gamma_{T_{n}^{2}}^{u_{2}^{2}} \phi_{1}-\widehat{\Gamma}_{2} \phi_{1}\right\|_{L^{2}}+\left\|\Gamma_{T_{n}^{1}}^{u_{n}^{1}} \alpha \phi_{l}-\widehat{\Gamma}_{1} \alpha \phi_{l}\right\|_{L^{2}}<\varepsilon
$$

Thanks to (39), there exist $K_{1}, K_{2}, K_{3}>0$ such that for every $\varepsilon>0$, there exist $T>0$ and $u \in L^{2}((0, T), \mathbb{R})$ such that $\left\|\Gamma_{T}^{u} \phi_{1}-\widetilde{\Gamma}_{N_{1}} \phi_{1}\right\|_{L^{2}}<\varepsilon$ and satisfying (42). The relation (36) and the triangular inequality achieve the claim.

1) (e) Approximate controllability with respect to the $L^{2}$-norm. Let $\psi \in \mathscr{H}$ and $\widehat{\Gamma} \in U(\mathscr{H})$.

Claim. There exist $K_{1}, K_{2}, K_{3}>0$ such that for every $\varepsilon>0$, there exist $T>0$ and $u \in L^{2}((0, T), \mathbb{R})$ such that $\left\|\Gamma_{T}^{u} \psi-\widehat{\Gamma} \psi\right\|_{L^{2}}<\varepsilon$ and satisfying (42).

We assume that $\|\psi\|_{L^{2}}=1$ but the same proof is also valid in the general case. We consider the unitary propagator $\widetilde{\Gamma}_{t}^{u}$ describing the reversed dynamics of the (BSE) introduced in Remark 4.3. We also recall the validity of the relation (25). Now, the results from [Cha12], which are adopted in the point 1) (c), are also valid for the reversed dynamics. Thus, such as in 1) (d), it is also true that, for every $\widehat{\Gamma} \in U(\mathscr{H})$, there exist $K_{1}, K_{2}, K_{3}>0$ such that for every $\varepsilon>0$, there exist $T>0$ and $u \in L^{2}((0, T), \mathbb{R})$ such that the relations $(42)$ are satisfied and $\left\|\widetilde{\Gamma}_{T}^{u} \phi_{1}-\widehat{\Gamma} \phi_{1}\right\|_{L^{2}}<\varepsilon$. By keeping in mind that $\widetilde{\Gamma}_{T}^{u}=\left(\Gamma_{T}^{u}\right)^{-1}$ and $\left\|\Gamma_{T}^{u}\right\| \|=1$, we have

$$
\left\|\phi_{1}-\Gamma_{T}^{u} \widehat{\Gamma} \phi_{1}\right\|_{L^{2}}=\left\|\Gamma_{T}^{u} \widetilde{\Gamma}_{T}^{u} \phi_{1}-\Gamma_{T}^{u} \widehat{\Gamma} \phi_{1}\right\|_{L^{2}} \leq\left\|\mid \Gamma_{T}^{u}\right\|\left\|\widetilde{\Gamma}_{T}^{u} \phi_{1}-\widehat{\Gamma} \phi_{1}\right\|_{L^{2}}<\varepsilon
$$

The last relation guarantees that, for every $\psi \in \mathscr{H}$ such that $\|\psi\|_{L^{2}}=1$, there exist $T_{1}>0$ and $u_{1} \in L^{2}\left(\left(0, T_{1}\right), \mathbb{R}\right)$ such that

$$
\left\|\Gamma_{T_{1}}^{u_{1}} \psi-\phi_{1}\right\|_{L^{2}}<\varepsilon .
$$

Now, 1) (d) ensures that, for every $\widehat{\Gamma} \in U(\mathscr{H})$, there exist $T_{2}>0$ and $u_{2} \in L^{2}\left(\left(0, T_{2}\right), \mathbb{R}\right)$ such that

$$
\left\|\Gamma_{T_{2}}^{u_{2}} \phi_{1}-\widehat{\Gamma} \psi\right\|_{L^{2}}<\varepsilon
$$

The chosen controls $u_{1}$ and $u_{2}$ satisfy (42). In conclusion, the claim is proved as

$$
\left\|\Gamma_{T_{2}}^{u_{2}} \Gamma_{T_{1}}^{u_{1}} \psi-\widehat{\Gamma} \psi\right\|_{L^{2}} \leq\left\|\Gamma_{T_{2}}^{u_{2}} \Gamma_{T_{1}}^{u_{1}} \psi-\Gamma_{T_{2}}^{u_{2}} \phi_{1}\right\|_{L^{2}}+\left\|\Gamma_{T_{2}}^{u_{2}} \phi_{1}-\widehat{\Gamma} \psi\right\|_{L^{2}}<2 \varepsilon .
$$

1) (f) Global approximate controllability in higher regularity norms. Let $\psi \in H_{\mathscr{G}}^{s}$ with $s \in$ $\left[s_{1}, s_{1}+2\right)$ and $s_{1} \in \mathbb{N}^{*}$. Let $\widehat{\Gamma} \in U(\mathscr{H})$ be such that $\widehat{\Gamma} \psi \in H_{\mathscr{G}}^{s}$ and $B: H_{\mathscr{G}}^{s_{1}} \longrightarrow H_{\mathscr{G}}^{s_{1}}$.

Claim. There exist $T>0$ and $u \in L^{2}((0, T), \mathbb{R})$ such that $\left\|\Gamma_{T}^{u} \psi-\widehat{\Gamma} \psi\right\|_{(s)}<\varepsilon$. 
We notice that the operator $-i(A+u(t) B-i c)$ is dissipative in $H_{\mathscr{G}}^{s_{1}}$ for $c:=\|u\|_{L^{\infty}((0, T), \mathbb{R})} \mid\|B\| \|_{\left(s_{1}\right)}$. Indeed, for every $\lambda>0$ and $\psi \in H_{\mathscr{G}}^{s_{1}+2}$, we have

$$
\|(\lambda+i(A+u(t) B-i c)) \psi\|_{\left(s_{1}\right)} \geq\|(\lambda+c+i A) \psi\|_{\left(s_{1}\right)}-\|u\|_{L^{\infty}((0, T), \mathbb{R})}\|B\|_{\left(s_{1}\right)}\|\psi\|_{\left(s_{1}\right)} .
$$

Now, the operator $A$ with domain $H_{\mathscr{G}}^{s_{1}+2}$ is self-adjoint in the Hilbert space $H_{\mathscr{G}}^{s_{1}}$ and we have the inequality $\|(\lambda+c+i A) \psi\|_{\left(s_{1}\right)} \geq(\lambda+c)\|\psi\|_{\left(s_{1}\right)}$. By recalling that $c=\|u\|_{L^{\infty}((0, T), \mathbb{R})}\|B\| \|_{\left(s_{1}\right)}$, we obtain

$$
\|(\lambda-i(-A+u(T-t) B-i c)) \psi\|_{\left(s_{1}\right)} \geq(\lambda+c)\|\psi\|_{\left(s_{1}\right)}-c\|\psi\|_{\left(s_{1}\right)}=\lambda\|\psi\|_{\left(s_{1}\right)} .
$$

Thus, $-i(A+u(t) B-i c)$ is dissipative and the Kato-Rellich's Theorem yields that it is also maximal dissipative. We consider the propagation of regularity developed by Kato in the work [Kat53]. Let $\lambda>c$ and $\widehat{H}_{\mathscr{G}}^{s_{1}+2}:=D\left(A^{\frac{s_{1}}{2}}(i \lambda-A)\right) \equiv H_{\mathscr{G}}^{s_{1}+2}$. We know that $B: \widehat{H}_{\mathscr{G}}^{s_{1}+2} \subset H_{\mathscr{G}}^{s_{1}} \rightarrow H_{\mathscr{G}}^{s_{1}}$ and the arguments of [Duc20, Remark 2.1] imply that $B \in L\left(\widehat{H}_{\mathscr{G}}^{s_{1}+2}, H_{\mathscr{G}}^{s_{1}}\right)$. For $T>0$ and $u \in B V((0, T), \mathbb{R})$, we have

$$
M:=\sup _{t \in[0, T]}\left\|(i \lambda-A-u(t) B)^{-1}\right\|_{L\left(H_{\mathscr{G}}^{s_{1}}, \widehat{H}_{\mathscr{G}}^{s_{1}+2}\right)}<+\infty .
$$

We know $\|k+f(\cdot)\|_{B V((0, T), \mathbb{R})}=\|f\|_{B V((0, T), \mathbb{R})}$ for $f \in B V((0, T), \mathbb{R})$ and $k \in \mathbb{R}$. Equivalently,

$$
N:=\|i \lambda-A-u(\cdot) B\|_{B V\left([0, T], L\left(\widehat{H}_{\mathscr{G}}^{s_{1}+2}, H_{\mathscr{G}}^{s_{1}}\right)\right)}=\|u\|_{B V(T)}\|B\|_{L\left(\widehat{H}_{\mathscr{G}}^{s_{1}+2}, H_{\mathscr{G}}^{s_{1}}\right)}<+\infty .
$$

We call $C_{1}:=\|\| A(A+u(T) B-i \lambda)^{-1} \|_{\left(s_{1}\right)}<\infty$ and $U_{t}^{u}$ the propagator generated by $A+u B-i c$ such that $U_{t}^{u} \psi=e^{-c t} \Gamma_{t}^{u} \psi$. Thanks to [Kat53, Section 3.10], for every $\psi \in H_{\mathscr{G}}^{s_{1}+2}$, it follows

$$
\left\|(A+u(T) B-i \lambda) U_{t}^{u} \psi\right\|_{\left(s_{1}\right)} \leq M e^{M N}\|(A-i \lambda) \psi\|_{\left(s_{1}\right)} \quad \Longrightarrow \quad\left\|\Gamma_{T}^{u} \psi\right\|_{\left(s_{1}+2\right)} \leq C_{1} M e^{M N+c T}\|\psi\|_{\left(s_{1}+2\right)} .
$$

For every $T>0, u \in B V((0, T), \mathbb{R})$ and $\psi \in H_{\mathscr{G}}^{s_{1}+2}$, there exists $C=C(K)>0$ depending on $K=$ $\left(\|u\|_{B V(T)},\|u\|_{L^{\infty}((0, T), \mathbb{R})}, T\|u\|_{L^{\infty}((0, T), \mathbb{R})}\right)$ such that

$$
\left\|\Gamma_{T}^{u} \psi\right\|_{\left(s_{1}+2\right)} \leq C\|\psi\|_{\left(s_{1}+2\right)} .
$$

Now, we notice that, for every $\psi \in H_{\mathscr{G}}^{6}$, we have $\|A \psi\|_{L^{2}}^{2} \leq\|\psi\|_{L^{2}}\left\|A^{2} \psi\right\|_{L^{2}}$ from the Cauchy-Schwarz inequality and there exists $C_{2}>0$ such that $\left\|A^{2} \psi\right\|_{L^{2}}^{4} \leq\|A \psi\|_{L^{2}}^{2}\left\|A^{3} \psi\right\|_{L^{2}}^{2} \leq C_{2}\|\psi\|_{L^{2}}\left\|A^{3} \psi\right\|_{L^{2}}^{3}$. By following the same idea, for every $\psi \in H_{\mathscr{G}}^{s_{1}+2}$, there exist $m_{1}, m_{2} \in \mathbb{N}^{*}$ and $C_{3}, C_{4}>0$ such that

$$
\left\|A^{\frac{s}{2}} \psi\right\|_{L^{2}}^{m_{1}+m_{2}} \leq C_{3}\|\psi\|_{L^{2}}^{m_{1}}\left\|A^{\frac{s_{1}+2}{2}} \psi\right\|_{L^{2}}^{m_{2}} \quad \Longrightarrow \quad\|\psi\|_{(s)}^{m_{1}+m_{2}} \leq C_{4}\|\psi\|_{L^{2}}^{m_{1}}\|\psi\|_{\left(s_{1}+2\right)}^{m_{2}}
$$

In conclusion, the point 1) (e), the relation (43) and the relation (44) ensure the claim.

1) (g) Conclusion. Let $d$ be defined in Assumptions $\operatorname{II}(\eta, \tilde{d})$. If $d<2$, then $B: H_{\mathscr{G}}^{2} \rightarrow H_{\mathscr{G}}^{2}$ and the global approximate controllability is verified in $H_{\mathscr{G}}^{d+2}$ since $d+2<4$. If $d \in[2,5 / 2)$, then $B: H^{d_{1}} \rightarrow H^{d_{1}}$ with $d_{1} \in(d, 5 / 2)$ from Assumptions $\operatorname{II}(\eta, \tilde{d})$. Now, $H_{\mathscr{G}}^{d_{1}}=H^{d_{1}} \cap H_{\mathscr{G}}^{2}$, thanks to [Duc18, Proposition 4.2], and $B: H_{\mathscr{G}}^{2} \rightarrow H_{\mathscr{G}}^{2}$ implies $B: H_{\mathscr{G}}^{d_{1}} \rightarrow H_{\mathscr{G}}^{d_{1}}$. The global approximate controllability is verified in $H_{\mathscr{G}}^{d+2}$ since $d+2<d_{1}+2$. If $d \in[5 / 2,7 / 2)$, then $B: H_{\mathcal{N} \mathcal{K}}^{d_{1}} \rightarrow H_{\mathcal{N} \mathcal{K}}^{d_{1}}$ for $d_{1} \in(d, 7 / 2)$ and $H_{\mathscr{G}}^{d_{1}}=H_{\mathcal{N} \mathcal{K}}^{d_{1}} \cap H_{\mathscr{G}}^{2}$ from [Duc18, Proposition 4.2]. Now, $B: H_{\mathscr{G}}^{2} \rightarrow H_{\mathscr{G}}^{2}$ that implies $B: H_{\mathscr{G}}^{d_{1}} \rightarrow H_{\mathscr{G}}^{d_{1}}$. The global approximate controllability is verified in $H_{\mathscr{G}}^{d+2}$ since $d+2<d_{1}+2$.

2) Generalization. Let $(A, B)$ do not admit a non-degenerate chain of connectedness. We decompose

$$
A+u(\cdot) B=\left(A+u_{0} B\right)+u_{1}(\cdot) B, \quad u_{0} \in \mathbb{R}, \quad u_{1} \in L^{2}((0, T), \mathbb{R}) .
$$

We notice that, if $(A, B)$ satisfies Assumptions $\mathrm{I}(\eta)$ and Assumptions $\operatorname{II}(\eta, \tilde{d})$ for $\eta>0$ and $\tilde{d} \geq 0$, then [Duc18, Lemma C.2 \& Lemma C.3] are valid. We consider $u_{0}$ belonging to the neighborhoods provided by [Duc18, Lemma C.2 \& Lemma C.3] and we denote $\left(\phi_{k}^{u_{0}}\right)_{k \in \mathbb{N}^{*}}$ a Hilbert basis of $\mathscr{H}$ made by eigenfunctions of $A+u_{0} B$. The steps of the point 1) can be repeated by considering the sequence $\left(\phi_{k}^{u_{0}}\right)_{k \in \mathbb{N}^{*}}$ instead of $\left(\phi_{k}\right)_{k \in \mathbb{N}^{*}}$ and the spaces $D\left(\left|A+u_{0} B\right|^{\frac{s_{1}}{2}}\right)$ in substitution of $H_{\mathscr{G}}^{s_{1}}$ with $s_{1}>0$. Thanks to the mentioned results, the claim is proved since $\left(A+u_{0} B, B\right)$ admits a non-degenerate chain of connectedness and $\| \mid A+$ $\left.u_{0} B\right|^{\frac{s_{1}}{2}} \cdot\left\|_{L^{2}} \asymp\right\| \cdot \|_{\left(s_{1}\right)}$ with $s_{1} \in[s, s+2), s=2+d$ and $d$ from Assumptions $\operatorname{II}(\eta, \tilde{d})$. 
By referring to Remark 4.3, we consider the reversed dynamics of the (BSE) and the unitary propagator $\widetilde{\Gamma}_{t}^{u}$ generated by the time-dependent Hamiltonian $-A-u(T-t) B$ with $u \in L^{2}((0, T), \mathbb{R})$. We also recall the validity of the relation (25). In the following corollary, we present the global approximate controllability for the reversed dynamics.

Corollary A.3. Let $(A, B)$ satisfy Assumptions $I(\eta)$ and Assumptions $I I(\eta, \tilde{d})$ for $\eta>0$ and $\widetilde{d} \geq 0$. Let $s=2+d$ be defined by $d$ from Assumptions $I I(\eta, \widetilde{d})$. For every $\psi \in H_{\mathscr{G}}^{s}, \widehat{\Gamma} \in U(\mathscr{H})$ such that $\widehat{\Gamma} \psi \in H_{\mathscr{G}}^{s}$ and $\varepsilon>0$, there exist $T>0$ and $u \in L^{2}((0, T), \mathbb{R})$ such that $\left\|\widehat{\Gamma} \psi-\widetilde{\Gamma}_{T}^{u} \psi\right\|_{(s)}<\varepsilon$.

Proof. First, we consider $K_{1}, K_{2}, K_{3}>0$ such that Proposition A.2 holds with times and controls satisfying the identities (42). Second, the point 1) (f) of the proof of Proposition A.2 is also valid for the propagator $\widetilde{\Gamma}_{T}^{u}$ as the operator $i(A+u(T-t) B+i c)$ with $c:=\|u\|_{L^{\infty}((0, T), \mathbb{R})}\|B\|_{\left(s_{1}\right)}$ is maximal dissipative. By interpolation, there exists a constant $C>0$ depending on $K_{1}, K_{2}$ and $K_{3}$ such that $\left\|\widetilde{\Gamma}_{T}^{u}\right\|_{(s)} \leq C$. Third, for every $\psi \in \mathscr{H}$ and $\widehat{\Gamma} \in U(\mathscr{H})$, we call $\widetilde{\psi}=\widehat{\Gamma} \psi$ and $\widetilde{\Gamma}=(\widehat{\Gamma})^{-1} \in U(\mathscr{H})$. For every $\varepsilon>0$, we set $\widetilde{\varepsilon}=\varepsilon C^{-1}$. Proposition A.2 ensures the existence of $T>0$ and $u \in L^{2}((0, T), \mathbb{R})$ such that $\left\|\Gamma_{T}^{u} \widetilde{\psi}-\widetilde{\Gamma} \widetilde{\psi}\right\|_{(s)}<\widetilde{\varepsilon}$ and the identities $(42)$ are verified. In conclusion, as $\widetilde{\Gamma}_{T}^{u}=\left(\Gamma_{T}^{u}\right)^{-1}$ and $\widetilde{\Gamma} \widetilde{\psi}=\psi$,

$$
\left\|\widetilde{\Gamma}_{T}^{u} \psi-\widehat{\Gamma} \psi\right\|_{(s)}=\left\|\widetilde{\Gamma}_{T}^{u} \psi-\widetilde{\Gamma}_{T}^{u} \Gamma_{T}^{u} \widehat{\Gamma} \psi\right\|_{(s)} \leq\left\|\widetilde{\Gamma}_{T}^{u}\right\|_{(s)}\left\|\psi-\Gamma_{T}^{u} \widehat{\Gamma} \psi\right\|_{(s)} \leq C\left\|\widetilde{\Gamma} \widetilde{\psi}-\Gamma_{T}^{u} \widetilde{\psi}\right\|_{(s)}<C \widetilde{\varepsilon}=\varepsilon .
$$

\section{B Appendix: Some diophantine approximation results}

For $x \in \mathbb{R}$, we denote $E(x)$ the closest integer number to $x,\|x\|=\min _{z \in \mathbb{Z}}|x-z|$ and $F(x)=x-E(x)$. We notice $|F(x)|=\|x \mid\|$ and $-\frac{1}{2} \leq F(z) \leq \frac{1}{2}$. Let $\left\{L_{j}\right\}_{j \leq N} \in\left(\mathbb{R}^{+}\right)^{N}$ and $i \leq N$. We also define

$$
n(x):=E\left(x-\frac{1}{2}\right), \quad r(x):=F\left(x-\frac{1}{2}\right), \quad d(x):=\left\|x-\frac{1}{2}\right\|, \quad \widetilde{m}^{i}(x):=n\left(\frac{L_{i}}{\pi} x\right) .
$$

In this appendix, we pursue [Duc18, Appendix A], which is based on the techniques from [DZ06, Appendix A].

Lemma B.1. Let $\left\{L_{k}\right\}_{k \leq N} \subset \mathbb{R}^{+}, I_{1} \subseteq\{1, \ldots, N\}, I_{2}:=\{1, \ldots, N\} \backslash I_{1}$ and

$$
a(\cdot):=\prod_{i \in I_{2}}\left|\sin \left((\cdot) L_{i}\right)\right| \sum_{i \in I_{1}} \prod_{\substack{j \in I_{1} \\ j \neq i}}\left|\cos \left((\cdot) L_{j}\right)\right|+\prod_{i \in I_{1}}\left|\cos \left((\cdot) L_{i}\right)\right| \sum_{\substack{i \in I_{2} \\ j \in I_{2} \\ j \neq i}}\left|\sin \left((\cdot) L_{j}\right)\right| \cdot
$$

Let $\left\{\widetilde{L}_{j}\right\}_{j \leq N} \subset \mathbb{R}^{+}$be such that $\widetilde{L}_{j}=2 L_{j}$ when $j \in I_{1}$, while $\widetilde{L}_{j}=L_{j}$ when $j \in I_{2}$. There exists $C>0$ such that, for every $x \in \mathbb{R}$, there holds

$$
a(x) \geq C \min \left(\min _{i \leq N} \prod_{j \neq i}\left\|\left(\widetilde{m}^{i}(x)+\frac{1}{2}\right) \frac{\widetilde{L}_{j}}{L_{i}}\right\|, \min _{i \leq N} \prod_{j \neq i}\left\|m^{i}(x) \frac{\widetilde{L}_{j}}{L_{i}}\right\|\right) .
$$

Proof. From [DZ06, relation (A.3)], for every $x \in \mathbb{R}$, there follows

$$
2 d(x) \leq|\cos (\pi x)| \leq \pi d(x) .
$$

As $2 d\left(\left(\widetilde{m}^{i}(x)+\frac{1}{2}\right) \frac{L_{j}}{L_{i}}\right) \leq\left|\cos \left(\left(\widetilde{m}^{i}(x)+\frac{1}{2}\right) \frac{L_{j}}{L_{i}} \pi\right)\right|$ and $\widetilde{m}^{i}(x)+\frac{1}{2}=\frac{L_{i}}{\pi} x-r\left(\frac{L_{i}}{\pi} x\right)$ for $x \in \mathbb{R}$ and $i, j \leq N$,

$$
2 d\left(\left(\widetilde{m}^{i}(x)+\frac{1}{2}\right) \frac{L_{j}}{L_{i}}\right) \leq\left|\cos \left(L_{j} x\right)\right|+\left|\sin \left(\pi \frac{L_{j}}{L_{i}}\left|r\left(\frac{L_{i}}{\pi} x\right)\right|\right)\right| .
$$

Now, $|\sin (\pi|r(\cdot)|)| \leq \pi|||r(\cdot)||| \leq \pi|r(\cdot)|=\pi d(\cdot) \leq \frac{\pi}{2}|\cos (\pi(\cdot))|$ thanks to [DZ06, relation (A.3)] and (45). For every $x \in \mathbb{R}$, it holds

$$
\left|\sin \left(\pi \frac{L_{j}}{L_{i}}\left|r\left(\frac{L_{i}}{\pi} x\right)\right|\right)\right| \leq \pi \frac{L_{j}}{L_{i}}\left|r\left(\frac{L_{i}}{\pi} x\right)\right| \leq \frac{\pi L_{j}}{2 L_{i}}\left|\cos \left(L_{i} x\right)\right| .
$$

From (46) and (47), there exists $C_{1}>0$ such that, for every $i, j \leq N$,

$$
2 d\left(\left(\widetilde{m}^{i}(x)+\frac{1}{2}\right) \frac{L_{j}}{L_{i}}\right) \leq\left|\cos \left(L_{j} x\right)\right|+\frac{\pi L_{j}}{2 L_{i}}\left|\cos \left(L_{i} x\right)\right|, \quad \forall x \in \mathbb{R}^{+},
$$




$$
\Longrightarrow \quad C_{1} \prod_{\substack{j \in I_{1} \\ j \neq i}} d\left(\left(\widetilde{m}^{i}(x)+\frac{1}{2}\right) \frac{L_{j}}{L_{i}}\right) \leq \prod_{\substack{j \in I_{1} \\ j \neq i}}\left|\cos \left(L_{j} x\right)\right|+\left|\cos \left(L_{i} x\right)\right| .
$$

From [DZ06, relation (A.3)], as done in (46) and (47), there exists $C_{2}>0$ such that

$$
\begin{gathered}
2\left\|\left|\left(\widetilde{m}^{i}(x)+\frac{1}{2}\right) \frac{L_{j}}{L_{i}} \| \leq\right| \sin \left(L_{j} x\right)\left|+\frac{\pi L_{j}}{2 L_{i}}\right| \cos \left(L_{i} x\right) \mid, \quad \forall x \in \mathbb{R},\right. \\
\Longrightarrow \quad C_{2} \prod_{\substack{j \in I_{1} \\
j \neq i}} d\left(\left(\widetilde{m}^{i}(x)+\frac{1}{2}\right) \frac{L_{j}}{L_{i}}\right) \prod_{\substack{j \in I_{2} \\
j \neq i}}\left\|\left|\left(\widetilde{m}^{i}(x)+\frac{1}{2}\right) \frac{L_{j}}{L_{i}} \| \leq \prod_{\substack{j \in I_{2} \\
j \neq i}}\right| \sin \left(L_{j} x\right)\left|\prod_{\substack{j \in I_{1} \\
j \neq i}}\right| \cos \left(L_{j} x\right)|+| \cos \left(L_{i} x\right) \mid .\right.
\end{gathered}
$$

Now, it is satisfied $d(x)=\left\|\frac{1}{2}(2 x-1)\right\| \geq \frac{1}{2}\|2 x-1\|=\frac{1}{2}\|2 x\|$ for every $x \in \mathbb{R}$ and then we have $d\left(\left(\widetilde{m}^{i}(x)+\frac{1}{2}\right) \frac{L_{j}}{L_{i}}\right) \geq \frac{1}{2}\left\|\left(\widetilde{m}^{i}(x)+\frac{1}{2}\right) \frac{2 L_{j}}{L_{i}}\right\|$, which imply

$$
C_{2} \prod_{\substack{j \leq N \\ j \neq i}} \frac{1}{2}\left\|\left|\left(\widetilde{m}^{i}(\cdot)+\frac{1}{2}\right) \frac{\widetilde{L}_{j}}{L_{i}} \| \leq a(\cdot)+\right| \cos \left(L_{i}(\cdot)\right) \mid .\right.
$$

Equivalently, from the proof of [DZ06, Proposition A.1], for every $x \in \mathbb{R}$,

$$
\begin{gathered}
2\left\|m^{i}(x) \frac{L_{j}}{L_{i}}\right\| \leq\left|\sin \left(L_{j} x\right)\right|+\frac{\pi L_{j}}{2 L_{i}}\left|\sin \left(L_{i} x\right)\right|, \quad 2 d\left(m^{i}(x) \frac{L_{j}}{L_{i}}\right) \leq\left|\cos \left(L_{j} x\right)\right|+\frac{\pi L_{j}}{2 L_{i}}\left|\sin \left(L_{i} x\right)\right|, \\
\Longrightarrow \quad C_{2} \prod_{\substack{j \leq N \\
j \neq i}} \frac{1}{2}\left\|\left|m^{i}(\cdot) \frac{\widetilde{L}_{j}}{L_{i}} \| \leq a(\cdot)+\right| \sin \left(L_{i}(\cdot)\right) \mid .\right.
\end{gathered}
$$

The claim follows as [DZ06, Proposition A.1]. Indeed, if $\left(\lambda_{k}\right)_{k \in \mathbb{N}^{*}} \subset \mathbb{R}^{+}$is so that $a\left(\lambda_{k}\right) \stackrel{k \rightarrow \infty}{\longrightarrow} 0$, then there exist some $i_{0} \leq N$ such that $\left|\sin \left(\lambda_{k} L_{i_{0}}\right)\right| \stackrel{k \rightarrow \infty}{\longrightarrow} 0$ or $\left|\cos \left(\lambda_{k} L_{i_{0}}\right)\right| \stackrel{k \rightarrow \infty}{\longrightarrow} 0$. By considering (50) and (51) with $i=i_{0}$, we have

$$
z\left(\lambda_{k}\right):=\min \left(\min _{i \leq N} \prod_{j \neq i}\left\|\left(\widetilde{m}^{i}\left(\lambda_{k}\right)+\frac{1}{2}\right) \frac{\widetilde{L}_{j}}{L_{i}}\right\|, \min _{i \leq N} \prod_{j \neq i}\left\|m^{i}\left(\lambda_{k}\right) \frac{\widetilde{L}_{j}}{L_{i}}\right\|\right) \stackrel{k \longrightarrow \infty}{\longrightarrow} 0 .
$$

The lemma is proved since $z\left(\lambda_{k}\right)$ converges to 0 at least as fast as $a\left(\lambda_{k}\right)$ thanks to (50) and (51).

Proposition B.2. Let $\left\{L_{j}\right\}_{j \leq N} \subset \mathbb{R}, I_{1} \subseteq\{1, \ldots, N\}$ and $I_{2}:=\{1, \ldots, N\} \backslash I_{1}$. If $\left\{L_{j}\right\}_{j \leq N} \in \mathcal{A L}(N)$, then, for every $\epsilon>0$, there exists $C_{\epsilon}>0$ such that, for every $x>\max \left\{\pi / 2 L_{j}: j \leq N\right\}$, we have

$$
\prod_{j \in I_{2}}\left|\sin \left(x L_{j}\right)\right| \sum_{j \in I_{1}} \prod_{\substack{k \in I_{1} \\ k \neq j}}\left|\cos \left(x L_{k}\right)\right|+\prod_{j \in I_{1}}\left|\cos \left(x L_{j}\right)\right| \sum_{j \in I_{2}} \prod_{\substack{k \in I_{2} \\ k \neq j}}\left|\sin \left(x L_{k}\right)\right| \geq \frac{C_{\epsilon}}{x^{1+\epsilon}} .
$$

Proof. The claim is due to Lemma B.1 and to the Schmidt's Theorem [DZ06, Theorem A.8], which implies that, for every $\epsilon>0$ and $i \leq N$, there exist $C_{1}(i), C_{2}(i), C_{3}(i)>0$ such that, for every $x \in \mathbb{R}$,

$$
\prod_{\substack{j \leq N \\ j \neq i}}\left\|\left(\widetilde{m}^{i}(x)+\frac{1}{2}\right) \frac{\widetilde{L}_{j}}{L_{i}}\right\| \geq \frac{C_{1}(i)}{\left(2 \widetilde{m}^{i}(x)+1\right)^{1+\epsilon}} \geq \frac{C_{1}(i)}{\left(\frac{2 L_{i}}{\pi} x+1\right)^{1+\epsilon}} \geq \frac{C_{2}(i)}{x^{1+\epsilon}}
$$

and $\prod_{\substack{j \leq N \\ j \neq i}}\left\|m^{i}(x) \frac{\widetilde{L}_{j}}{L_{i}}\right\| \mid \geq C_{3}(i) x^{-1-\epsilon}$ for every $x>\frac{\pi}{2} \max \left\{1 / L_{j}: j \leq N\right\}$. The statement follows with $C_{\epsilon}:=\min \left(\min _{i \leq N} C_{2}(i), \min _{i \leq N} C_{3}(i)\right)$.

Corollary B.3. Let $\left\{L_{k}\right\}_{k \leq N} \in \mathcal{A L}(N)$ with $N \in \mathbb{N}^{*}$. Let $\left\{\omega_{n}\right\}_{n \in \mathbb{N}^{*}}$ be the unbounded sequence of positive solutions of the equation

$$
\sum_{l \leq N} \sin \left(x L_{l}\right) \prod_{m \neq l} \cos \left(x L_{m}\right)=0, \quad x \in \mathbb{R} .
$$

For every $\epsilon>0$, there exists $C_{\epsilon}>0$ so that $\left|\cos \left(\omega_{n} L_{l}\right)\right| \geq \frac{C_{\epsilon}}{\omega_{n}^{1+\epsilon}}$ for every $l \leq N$ and $n \in \mathbb{N}$. 
Proof. If there exists $\left\{\omega_{n_{k}}\right\}_{k \in \mathbb{N}^{*}}$, subsequence of $\left\{\omega_{n}\right\}_{n \in \mathbb{N}^{*}}$, such that $\mid \cos \left(L_{j} \omega_{n_{k}}\right) \stackrel{k \rightarrow \infty}{\longrightarrow} 0$ for some $j \leq N$, then there exists $i \leq N$ such that $i \neq j$ and $\left|\cos \left(L_{i} \omega_{n_{k}}\right)\right| \stackrel{k \rightarrow \infty}{\longrightarrow} 0$ thanks to (53). The last convergence is at least as fast as the first one. From (48), we have $\prod_{j \neq i} d\left(\left(\widetilde{m}^{i}\left(\omega_{n_{k}}\right)+\frac{1}{2}\right) \frac{L_{j}}{L_{i}}\right) \stackrel{k \rightarrow \infty}{\longrightarrow} 0$ at least as fast as $\left|\cos \left(L_{j} \omega_{n_{k}}\right)\right| \stackrel{k \rightarrow \infty}{\longrightarrow} 0$. As in the proof of Lemma B.1, there exists $C_{2}>0$ so that

$$
C_{2}\left|\cos \left(L_{i} \omega_{n}\right)\right| \geq \prod_{j \neq i} d\left(\left(\widetilde{m}^{i}\left(\omega_{n}\right)+\frac{1}{2}\right) \frac{L_{j}}{L_{i}}\right)=\prod_{j \neq i}\left\|\frac{1}{2}\left(\left(\widetilde{m}^{i}\left(\omega_{n}\right)+\frac{1}{2}\right) \frac{2 L_{j}}{L_{i}}-1\right)\right\| .
$$

The last identity and the techniques leading to the equation (52) achieve the claim.

\section{References}

[Alt02] C. Altafini. Controllability of quantum mechanical systems by root space decomposition. $J$. Math. Phys., 43(5):2051-2062, 2002.

[AM01] S. Avdonin and W. Moran. Ingham-type inequalities and Riesz bases of divided differences. Int. J. Appl. Math. Comput. Sci., 11(4):803-820, 2001. Mathematical methods of optimization and control of large-scale systems (Ekaterinburg, 2000).

[BC06] Karine Beauchard and Jean-Michel Coron. Controllability of a quantum particle in a moving potential well. J. Funct. Anal., 232(2):328-389, 2006.

[BCMS12] U. V. Boscain, F. Chittaro, P. Mason, and M. Sigalotti. Adiabatic control of the Schrödinger equation via conical intersections of the eigenvalues. IEEE Trans. Automat. Control, 57(8):1970-1983, 2012.

[BCS14] U. Boscain, M. Caponigro, and M. Sigalotti. Multi-input Schrödinger equation: controllability, tracking, and application to the quantum angular momentum. J. Differential Equations, 256(11):3524-3551, 2014.

[BdCC13] N. Boussaï d, M. Caponigro, and T. Chambrion. Weakly coupled systems in quantum control. IEEE Trans. Automat. Control, 58(9):2205-2216, 2013.

[Bea05] K. Beauchard. Local controllability of a 1-D Schrödinger equation. J. Math. Pures Appl. (9), 84(7):851-956, 2005.

[BGRS15] U. Boscain, J. P. Gauthier, F. Rossi, and M. Sigalotti. Approximate controllability, exact controllability, and conical eigenvalue intersections for quantum mechanical systems. Comm. Math. Phys., 333(3):1225-1239, 2015.

[BKL02] C. Baiocchi, V. Komornik, and P. Loreti. Ingham-Beurling type theorems with weakened gap conditions. Acta Math. Hungar., 97(1-2):55-95, 2002.

[BL10] K. Beauchard and C. Laurent. Local controllability of 1D linear and nonlinear Schrödinger equations with bilinear control. J. Math. Pures Appl. (9), 94(5):520-554, 2010.

[BL17] K. Beauchard and C. Laurent. Bilinear control of high frequencies for a 1D Schrödinger equation. Mathematics of Control, Signals, and Systems, 29(2), 2017.

[BLR92] C. Bardos, G. Lebeau, and J. Rauch. Sharp sufficient conditions for the observation, control, and stabilization of waves from the boundary. SIAM J. Control Optim., 30(5):1024-1065, 1992.

[BM08] L. Baudouin and A. Mercado. An inverse problem for Schrödinger equations with discontinuous main coefficient. Appl. Anal., 87(10-11):1145-1165, 2008.

[BMS82] J. M. Ball, J. E. Marsden, and M. Slemrod. Controllability for distributed bilinear systems. SIAM J. Control Optim., 20(4):575-597, 1982.

[Bur91] N. Burq. Contrôle de l'équation de Schrödinger en présence d'obstacles strictement convexes. In Journées "Équations aux Dérivées Partielles" (Saint Jean de Monts, 1991), pages Exp. No. XIV, 11. École Polytech., Palaiseau, 1991. 
[Cha12] T. Chambrion. Periodic excitations of bilinear quantum systems. Automatica J. IFAC, 48(9):2040-2046, 2012.

[Cor07] J. M. Coron. Control and nonlinearity, volume 136 of Mathematical Surveys and Monographs. American Mathematical Society, Providence, RI, 2007.

[Duc18] A. Duca. Bilinear quantum systems on compact graphs: well-posedness and global exact controllability. preprint: https://hal.archives-ouvertes.fr/hal-01830297, 2018.

[Duc19] A Duca. Controllability of bilinear quantum systems in explicit times via explicit control fields. To be published in International Journal of Control, 2019.

[Duc20] A. Duca. Simultaneous global exact controllability in projection of infinite 1D bilinear Schrödinger equations. Dynamics of Partial Differential Equations, 17(3):275-306, 2020.

[DZ06] R. Dáger and E. Zuazua. Wave propagation, observation and control in 1-d flexible multistructures, volume 50 of Mathématiques 8 Applications (Berlin) [Mathematics 8 Applications]. Springer-Verlag, Berlin, 2006.

[Kat53] T. Kato. Integration of the equation of evolution in a Banach space. J. Math. Soc. Japan, 5:208-234, 1953.

[Kuc04] P. Kuchment. Quantum graphs. I. Some basic structures. Waves Random Media, 14(1):S107S128, 2004. Special section on quantum graphs.

[Leb92] G. Lebeau. Contrôle de l'équation de Schrödinger. J. Math. Pures Appl. (9), 71(3):267-291, 1992.

[Lio83] J.-L. Lions. Contrôle des systèmes distribués singuliers, volume 13 of Méthodes Mathématiques de l'Informatique [Mathematical Methods of Information Science]. Gauthier-Villars, Montrouge, 1983.

[LT92] I. Lasiecka and R. Triggiani. Optimal regularity, exact controllability and uniform stabilization of Schrödinger equations with Dirichlet control. Differential Integral Equations, 5(3):521-535, 1992.

[Lue69] D. G. Luenberger. Optimization by vector space methods. John Wiley \& Sons, Inc., New York-London-Sydney, 1969.

[Mac94] E. Machtyngier. Exact controllability for the Schrödinger equation. SIAM J. Control Optim., 32(1):24-34, 1994.

[Mir09] M. Mirrahimi. Lyapunov control of a quantum particle in a decaying potential. Ann. Inst. H. Poincaré Anal. Non Linéaire, 26(5):1743-1765, 2009.

[MN15] M. Morancey and V. Nersesyan. Simultaneous global exact controllability of an arbitrary number of 1D bilinear Schrödinger equations. J. Math. Pures Appl. (9), 103(1):228-254, 2015.

[MOR08] A. Mercado, A. Osses, and L. Rosier. Inverse problems for the Schrödinger equation via Carleman inequalities with degenerate weights. Inverse Problems, 24(1):015017, 18, 2008.

[Mor14] M. Morancey. Simultaneous local exact controllability of 1D bilinear Schrödinger equations. Ann. Inst. H. Poincaré Anal. Non Linéaire, 31(3):501-529, 2014.

[Ner10] V. Nersesyan. Global approximate controllability for Schrödinger equation in higher Sobolev norms and applications. Ann. Inst. H. Poincaré Anal. Non Linéaire, 27(3):901-915, 2010.

[Sac00] Yu. L. Sachkov. Controllability of invariant systems on Lie groups and homogeneous spaces. J. Math. Sci. (New York), 100(4):2355-2427, 2000. Dynamical systems, 8.

[Tur00] G. Turinici. On the controllability of bilinear quantum systems. In Mathematical models and methods for ab initio quantum chemistry, volume 74 of Lecture Notes in Chem., pages 75-92. Springer, Berlin, 2000.

[You80] R. M. Young. An introduction to nonharmonic Fourier series, volume 93 of Pure and Applied Mathematics. Academic Press, Inc. [Harcourt Brace Jovanovich, Publishers], New York-London, 1980. 